\title{
Proceedings of the Canadian Society for Exercise Physiology Annual General Meeting - Zooming into the Future: Exercise Science in the Virtual Age
}

\author{
Committee Chair \\ Stephen Cheung, Brock University
}

\section{Committee Members}

Jason $\mathrm{Au}$, University of Waterloo

Jamie Burr, University of Guelph

Arthur Cheng, York University

Stephen Cheung, Brock University

Katharine Currie, Michigan State University

Michaela Devries-Aboud, University of Waterloo

Brendon Gurd, Queen's University

Tom Hazell, Wilfred Laurier University

Susanne Janisch, McMaster University

Panagiota (Nota) Klentrou, Brock University

Maureen MacDonald, McMaster University

Chris McGlory, Queen's University

Philip Millar, University of Guelph

Greg Pearcey, Northwestern University

Nick Ravanelli, Lakehead University

Daniel Santa Mina, University of Toronto

Martin Senechal, University of New Brunswick

Kyle Thompson, University of Guelph

Justine Tishinsky, University of Guelph

Thomas Tripp, University of Calgary

Charlotte Usselman, McGill University

Jennifer Williams, McMaster University

\section{Organizer}

Canadian Society for Exercise Physiology, Ottawa, Ontario

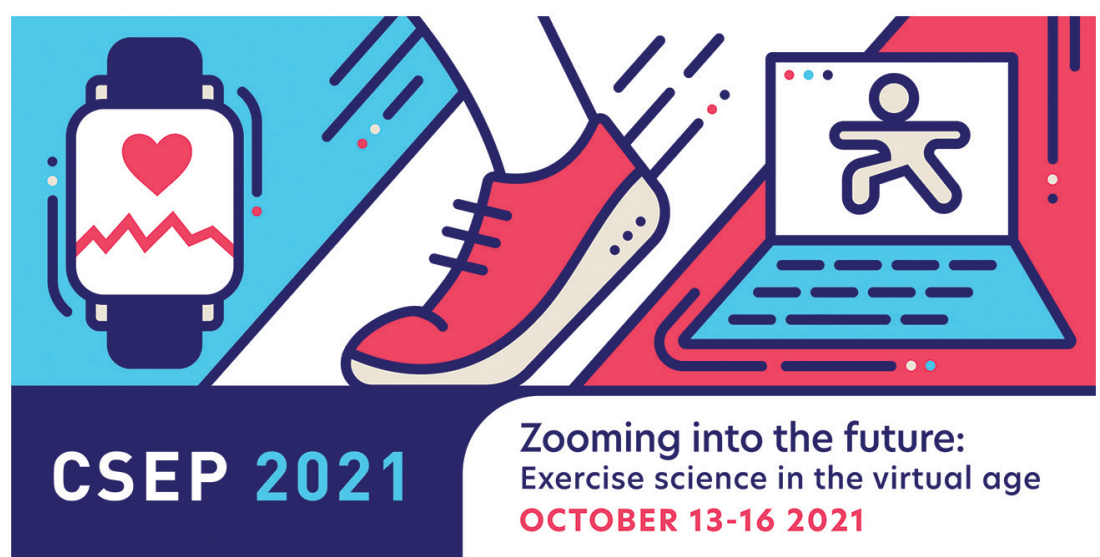




\section{Contents / Contenu}

Post-exercise muscle ischemia maintains central and peripheral fatigue following repeated isometric knee extensions to task failure: Preliminary findings

A qualitative study to examine the impact of on online resilience intervention for personal trainers delivered during the COVID-19 pandemic

An assessment of running power output measured with the Coros Pace 2 near maximal lactate steady state

Role of cardiac rehabilitation adherence on structural and functional brain health in ischemic heart disease patients

Repeated submaximal handgrip contractions alter cortical functional connectivity and response times with the contralateral hand

Similar rate of development of the $\dot{\mathrm{V}} \mathrm{O}_{2}$ slow component and peripheral fatigue across different exercise durations and intensities near the MLSS

Ketone gavage alters BACE1 content and activity in a brain region specific manner

Effects of high-intensity interval exercise on natural killer cell number and function in pre- and post-pubertal children

Moderate altitude reduces the power output at the maximal lactate steady state

Sex differences in skeletal muscle adaptations of middle-aged adults after 10 weeks of progressive resistance training

Consumption of a high-fat-high-sucrose diet partly prevents cardiac mechanical adaptations to aerobic and resistance exercise training

The effects of an acute bout of high-intensity interval exercise in the morning vs. evening on the magnitude of the morning blood pressure surge

Impact of high intensity interval training with blood flow restriction on aerobic and anaerobic performances in endurance-trained athletes: preliminary results

A multimodal intervention to improve brain function in older adults with mild cognitive impairment. Results from SYNERGIC.

Factors affecting walking compliance in patients with peripheral artery disease

Differential postprandial amino acid responses following the consumption of isonitrogenous doses of Greek Yogurt and Skim Milk

The effects of high intensity interval training on PTSD symptomology
Abel et al.

Adams et al. 
The Comparison of Muscle Function Loss of Respiratory Muscles and the Tibialis Anterior

Habitual cannabis users demonstrate altered cardiac mechanics at rest, but not during isometric handgrip exercise

NHL Combine fitness test results predict long-term career performance: a longitudinal 25-year analysis

Late effects of radiation therapy on skeletal muscle fibro/adipogenic progenitor and satellite cell crosstalk

The history dependence of torque following eccentric exercise-induced muscle weakness

Vestibular control of standing balance following 24-hour sleep deprivation

Ability of pre-season fitness to predict in-season on-ice performance for male adolescent ice-hockey athletes

Can high cardiorespiratory and musculoskeletal performance coincide? An examination in female collegiate soccer players

The relationship between single muscle fibre and voluntary rate of force development

Vertical force output does not govern sprint performance in female college soccer players

Frequency and time domain responses of vestibular-evoked balance reflexes following foot dorsum cooling

Implementation of Teleprehabilitation for Onco-Surgical Candidates during Covid-19 Pandemic: A Retrospective Pilot Cohort

Prehabilitation to Enhance Esophageal Cancer Care: Preoperative Functional Outcomes

A multi-method approach to adapt adverse event reporting guidelines to resistance training studies

Carbohydrate-energy replacement following low-volume high-intensity interval exercise blunts next-day glycemic control in women

Resistance exercise training induces load specific absolute muscle endurance gains in young women: a within-participant randomized controlled trial

The acute effects of post-exercise milk consumption on systemic inflammation after combined resistance and plyometric exercise in young females
Chan et al.

S50

Cheung et al.

Cohen et al.

Collao et al. 
A systematic review and meta-analysis of sex-based differences in

Hawley et al. 
Senescence-associated metabolomic profiling of skeletal muscle cells

Effects of lung volume on signal-averaged sympathetic transduction of blood pressure

The Effects of a Single Bout of Isometric Handgrip Exercise on Nighttime Blood Pressure in Young Normotensive Men and Women

The Effects of Exercise on Anthracyline Induced Cardiotoxicity in Breast Cancer Survivors

Muscle-strengthening activities influence body fat in both women and men living with obesity: Is sex influencing the association between MSA and health outcomes?

Training status is related to lactate stability at the maximal lactate steady state after mild elevation in carboxyhemoglobin

Sclerostin regulates exercise induced adaptations in energy expenditure and body composition in $\mathrm{C} 57 \mathrm{BL} / 6 \mathrm{~J}$ mice

A Pilot Study Investigating Problematic Exercise Behavior Patterns Amongst Varsity Level University Athletes

Children's adherence to 24-hour movement guidelines during the second wave of COVID-19: national survey

Sex differences in muscle metaboreflex activation following static handgrip exercise

Evaluating the relationships between bone-specific physical activity and nutrition behaviours and measures of bone mineral density in young men and women

Does aerobic fitness impact prolonged sitting-induced popliteal artery endothelial dysfunction?

Investigating physical activity intensity of virtual reality exergame in recreationally active young adults

Outcome domains measured in randomized controlled trials of physical activity for older adults: A rapid review

Estimation of the $\dot{\mathrm{VO}}_{2}$ mean response time utilizing two step transitions of different amplitudes preceding a ramp-incremental test

Ventilatory response of peripheral chemosensors to hypercapnia during exercise

The impact of abdominal body contouring surgery on physical function after a massive weight loss: a pilot non-randomized controlled trial
Kamal et al.

S60

Katerberg et al.

S60

Kathia et al.

S61

Kendall et al.

S61

Keshavarz et al.

S61

Kontro et al.

Kurgan et al.

Landolfi

Larouche et al.

Lee et al.

Levee et al.

Mackie et al. 
The heart rate at the respiratory compensation point is not a valid surrogate to derive the heart rate at the maximal metabolic steady state during endurance exercise

The effects of a gradual VCD-induced ovarian failure model of

The long-term effect of incident fractures on self-reported physical activity in adults

Examining cardiovascular risk metrics in osteoarthritic and non-osteoarthritic populations using CLSA data

HIIT me with your best shot: The role of exercise intensity on cognitive function throughout the lifespan

Regulation of mitochondrial function by the UPRmt transcriptional regulator ATF4

The Impact of Exercise Training on Muscle Sympathetic Nerve Activity: A Systematic Review and Meta-Analysis

Sulforaphane and Urolithin A exert time-dependent effects on mitochondrial protein signaling in $\mathrm{C} 2 \mathrm{C} 12$ myotubes

Reliability and validity of dietary assessment tools in a Canadian athlete population

Association between Exercise Levels and Physical Function after a 6-week Virtual Exercise Program for Breast Cancer Survivors Receiving Endocrine Therapy Treatment

Correlates of physical activity in children from families speaking non-official languages at home: a multi-site Canadian study

A novel AMPK activator stimulates autophagy signaling in dystrophic skeletal muscle

The influence of social engagement during exercise on associated cognitive and affective changes among older adult women

Sympathetic inhibition during mild and moderate dynamic leg exercise in healthy middle-aged subjects: contributing mechanisms

The Effect of Sex on Neurovascular Recovery from Acute Stress

Criterion-validity of the Physical Activity Vital Sign for estimating habitual moderate-to-vigorous physical activity in active younger and older adults
McLaughlin et al.

Mei et al. 
Sympathetic neurohemodynamic transduction is attenuated in older males independent of age-related declines in aerobic fitness

Acute Effects of Milk vs Carbohydrate on Bone Turnover Biomarkers Following Exercise in Young Adult Females

Fitness level- and sex-related differences in FMD responses

Effect of heat waves on 24-hour movement behaviour, thermal perception, and thirst in children and adults: a pilot study

Screening Varsity Athletes for Sleep Quality and Sleep-Associated Behaviours: A Pilot Study

Tafazzin deficiency attenuates maximal mitochondrial respiration in $\mathrm{C} 2 \mathrm{C} 12$ myotubes

Health-related criterion-referenced cut-points for cardiorespiratory fitness among youth: A systematic review

Prevalence and correlates of meeting the Canadian 24-Hour Movement Guidelines among a sample of Canadian parents during the COVID-19 pandemic

Muscle power mediates deficits in the tibia bone strength, area and mass in children with autism spectrum disorder

UW WELL-FIT Cancer Exercise Program: 20 Years and Thriving

The effects of chemoradiation and resistance wheel running on performance and muscle stem/progenitor cell populations in a pediatric mouse model of rhabdomyosarcoma

Airway size and its association with exertional dyspnoea in healthy males and females

Muscle quality and physical function in colon cancer patients who are complete adjuvant treatment: A pilot study

Evaluating the associations between osteoporosis health beliefs and bone-specific physical activity and nutrition behaviors in young adults

Physical activity and sedentary time in children with concussion in comparison to age-, sex-, and season-matched healthy controls

Wearing of a facemask in ice-hockey playing youth during the COVID-19 pandemic does not affect performance

A pea-powder supplement provided to female endurance athletes has potential to improve iron status
Petterson et al.

S70

Prowting et al.

S70

Rasica et al.

Ravanelli et al.

Rebello et al.

S71

Richards et al.

Rollo et al. 
MoveSTroNg at Home: A feasibility study of a model for remote delivery of functional strength and balance training combined with nutrition education for older pre-frail adults

Examining the impact of contraceptive hormones on the nitric oxide pathway in vascular endothelial cells

More frequent breaks in sedentary time is associated with better autonomic heart rate control in adults

Does adjusting for mechanical work affect sex differences in peripheral and central fatigue during repeated sprints?

Principles of exercise training in randomized controlled trials of cancers other than breast and prostate: An updated systematic review

Contralateral pain does not modulate corticospinal excitability or neuromuscular fatigue during repeated isometric knee extensions to task failure: Preliminary evidence

Higher body fat percentage in children with type 1 diabetes: a systematic review and meta-analysis
Wang et al.

S80

Williams et al.

S81

Wu et al.

S81

Yoon et al.

S81

Zadravec et al.

Zhang et al.

S82

Zheng et al.

S82 
Post-exercise muscle ischemia maintains central and peripheral fatigue following repeated isometric knee extensions to task failure: Preliminary findings

\author{
S. Abel, J. Zhang, M. Macphail, D. Iannetta, and S.J. Aboodarda \\ Faculty of Kinesiology, University of Calgary, Calgary, AB T2N 1N4, Canada.
}

Following exhaustive, high-intensity exercise, inhibitory group III/ IV afferent feedback maintained in the absence of central motor command may modulate neuromuscular fatigue recovery patterns. We hypothesize that accumulation of meltabilities in the fatigued muscles resulting from blood flow occlusion would prevent the recovery of central and peripheral fatigue measures and consequently maximal voluntary force output (MVC). Seven participants ( 2 females; $26 \pm 7$ years) performed repeated ( $6 \mathrm{~s}$ contraction: $4 \mathrm{~s}$ rest) unilateral isometric knee extensions [30\% of MVC force] to task failure. Neuromuscular fatigue assessments, consisting of a 5-s MVC with superimposed and resting femoral nerve electrical stimulations to assess central and peripheral fatigue, were performed at baseline, task failure (TF), as well as 30 and $60 \mathrm{~s}$ of recovery. Upon TF, participants' femoral artery and vein were fully occluded (OCCL) or not (CTRL) at TF for $30 \mathrm{~s}$. Preliminary results demonstrated that following task failure, MVC was lower in OCCL compared to CTRL at $30 \mathrm{~s}$ (vs. TF; CTRL: $+45.5 \pm 39.9 \%$; OCCL: $-10.7 \pm 25.9 \% ; P=0.036$ ) and $60 \mathrm{~s}$ (vs. TF; CTRL: $+50.5 \pm 33.7 \%$; OCCL: $+26.4 \pm 21.6 \% ; P=0.036$ ). Voluntary activation did not differ between conditions at $30 \mathrm{~s}$ (CTRL: $+13.8 \pm 18.5 \%$; OCCL: $-2.2 \pm 31.0 \% ; P=0.241$ ) or 60 s (CTRL: $12.9 \pm 15.3 \%$; OCCL: $13.9 \pm 13.0 \%$; $P=0.471)$. Resting twitch force was lower at $30 \mathrm{~s}(P=0.002)$ following OCCL $(-23.0 \pm 13.3 \%)$ compared to CTRL (+49.1 $\pm 49.3 \%)$. Our results indicate post-exercise ischemia prevented recovery of voluntary force output and central and peripheral fatigue. Maintained group III/IV afferent firing and inhibited clearance of accumulated metabolites in the exercised quadriceps muscles resulted in maintained depression of voluntary force output and central and peripheral fatigue, however, a larger sample size is required to confirm initial findings. (Funded by the Natural Sciences and Engineering Research Council of Canada)

A qualitative study to examine the impact of on online resilience intervention for personal trainers delivered during the COVID-19 pandemic

\section{B.P. Adams, ${ }^{1}$ R.E. Rhodes, ${ }^{1}$ C.M. Smart, ${ }^{2}$ and S.X. Liu ${ }^{1}$ \\ ${ }^{1}$ School of Exercise Science, Physical \& Health Education, University of Victoria, Victoria, BC V8W 2Y2, Canada. \\ ${ }^{2}$ Department of Psychology, University of Victoria, Victoria, BC V8W 2Y2, Canada.}

The COVID-19 pandemic has presented tremendous challenges (job losses, fitness facility closures, pivoting to the online space) for personal trainers. Currently there is a lack of resilience training programs for these health professionals to help them cope with the adversity they experienced working during the pandemic. This study aimed to 1) assess personal trainers' perceived impact of an online resiliency intervention targeting the domains of vision, collaboration and health and 2) evaluate intervention acceptability and satisfaction. Certified Personal Trainers were recruited online and through email marketing in B.C. The 10-week resilience intervention (The Resilient Trainer Program) started in April 2020. The intervention was developed based on the American Psychology Associations 10 strategies for building resilience. Participants received weekly online group coaching (60 minutes) and participated in a private Facebook group. 29 participants (27 female, 2 male) enrolled in the program with 20 participants $(69 \%)$ completing the program. Those who completed the program were invited to participate in semi- structured interviews following the intervention $(n=7)$. Interview questions were based on the Predictive 6 Factor Resilience Scale and focussed on the domains of vision, collaboration and health. Interview transcripts were thematically analyzed. The results of this study show that the online intervention strengthened perceptions of the collaboration, vision and health domains of resiliency. Specifically, collaboration was strengthened through increased social support while vision was strengthened through increased clarity on what is truly important. The perception of health was strengthened through spending more time in self-care activities and recognizing the need for rest. Participants found the intervention to be acceptable, however it was noted that more examples from a male perspective could be added. The results support moving to an effectiveness trial to examine the impact of the intervention on building resilience in Personal Trainers.

An assessment of running power output measured with the Coros Pace 2 near maximal lactate steady state

O.O. Ajayi, C.R. van Rassel, K.M. Sales, and M.J. MacInnis

Faculty of Kinesiology, University of Calgary, Calgary, AB T2N IN4, Canada.

Commercially available wearable technology may provide practical and accurate assessments of exercise intensity with respect to exercise thresholds. The purpose of this study was to determine whether the Coros Pace 2-a wrist-worn sports watch that provides a measure of running power output (PO) - can accurately detect changes in treadmill running speeds $(+5 \%$ and $-5 \%)$ relative to the maximal lactate steady state (MLSS). Thirteen male and female trained runners ( $30 \pm 5$ years; $57.0 \pm 5.7 \mathrm{~mL} \cdot \mathrm{kg}^{-1} \cdot \mathrm{min}^{-1}[$ mean $\pm S D]$ ) completed, on separate days, a maximal incremental treadmill test, three to four 30-minute constant-speed exercise tests to determine the PO associated with MLSS, and a repeat trial at MLSS. Constantspeed treadmill running $5 \%$ above and $5 \%$ below MLSS resulted in significantly higher $(217.1 \pm 33.3 \mathrm{~W} ; \mathrm{p}<0.001)$ and lower $(207.6 \pm$ $32.1 \mathrm{~W} ; \mathrm{p}<0.05)$ average running POs, respectively, compared to running PO at MLSS (212.2 $\pm 33.2 \mathrm{~W})$. The PO at MLSS was not significantly different than the PO during the repeat trial at MLSS (212.1 \pm $31.1 \mathrm{~W} ; \mathrm{p}>0.05$ ) and were strongly correlated (ICC $=0.99$ ). For each running speed, the average POs for the first and last 5 minutes were not significantly different $(\mathrm{p}>0.05)$. Running economy at MLSS was $15.7 \pm$ $1.7 \mathrm{~mL} \mathrm{O}_{2} / \mathrm{W}$. Based on these results, the running PO measured by the Coros Pace 2 on a treadmill was sensitive, stable, repeatable, and physiologically reasonable. Overall, this device seems suitable for measuring the intensity of exercise near the MLSS. (Supported by the Natural Sciences and Engineering Research Council of Canada (NSERC), NSERC CREATE We-TRAC training program, and Alberta Innovates.)

Role of cardiac rehabilitation adherence on structural and functional brain health in ischemic heart disease patients

\section{E.Y. Allison, ${ }^{1}$ J.K. Shoemaker, ${ }^{2}$ and B.K. Al-Khazraji ${ }^{1}$}

${ }^{I}$ Department of Kinesiology, Faculty of Science, McMaster University, Hamilton, ON, Canada ${ }^{2}$ School of Kinesiology, Faculty of Health Sciences, Western University, London, ON, Canada.

Ischemic heart disease (IHD) is associated with cerebrovascular and neurological complications. It is unknown whether adherence to standard cardiac rehabilitation (CR) programs improves cerebrovascular function and cognition in IHD patients. We tested the hypothesis that IHD patients enrolled in CR programs would see improvements in cerebrovascular, cognitive, and structural brain measures proportional to their CR adherence (total CR exercise sessions). Twenty-four older adults (62 \pm 8 years) enrolled in a 6-month CR program underwent 
cerebrovascular assessment (resting Middle Cerebral Artery [MCA] blood flow velocity as well as $\mathrm{CO}_{2}$ reactivity for MCA cerebral blood flow $\left[\mathrm{CBF}_{\text {reactivity }}\right], \mathrm{MCA}$ cross sectional area [CSA $\left.\mathrm{reactivity}_{\text {, }}\right]$ and MCA blood flow velocity $\left[\mathrm{VEL}_{\text {reactivity }}\right]$ ). Also, cognitive (Montreal Cognitive Assessment Test [MoCA], Trail Making A and B [TmA, TmB]), and brain imaging (cortical thickness) assessments were made prior to and following the 6-month CR program. $\triangle$ resting MCA velocity $(\mathrm{r}=0.031$, $\mathrm{p}=0.895), \Delta \mathrm{CBF}_{\text {reactivity }}(\mathrm{r}=-0.101, \mathrm{p}=0.797), \Delta \mathrm{CSA}_{\text {reactivity }}(\mathrm{r}=0.048$, $\mathrm{p}=0.88)$, and $\Delta \mathrm{VEL}_{\text {reactivity }}(\mathrm{r}=0.075, \mathrm{p}=0.791)$ did not associate with CR exercise sessions. Total CR exercise sessions correlated with $\Delta \operatorname{TmA}$ performance $(\mathrm{r}=-0.465, \mathrm{p}=0.029)$ but not $\Delta \mathrm{MoCA}(\mathrm{r}=0.082$, $\mathrm{p}=0.722)$ or $\Delta \operatorname{TmB}(\mathrm{r}=-0.238, \mathrm{p}=0.299)$. Cortical thinning in the left caudal anterior cingulate $(\mathrm{r}=-0.45, \mathrm{p}=0.072)$ and left insula $(\mathrm{r}=$ $-0.42, \mathrm{p}=0.092)$ had a non-significant negative association to total CR exercise sessions. Following 6-month CR, changes in one cognitive domain and a reduced rate of brain degradation in select regions were observed related to CR adherence in IHD. These data suggest that $\mathrm{CR}$ programs may improve brain outcomes in IHD although future studies should investigate the role of exercise adherence on brain health in a larger cohort of patients.

\section{Repeated submaximal handgrip contractions alter cortical functional connectivity and response times with the contralateral hand}

Justin W. Andrushko, ${ }^{1,2}$ Jacob M. Levenstein, ${ }^{2,3}$ Catharina Zich, ${ }^{2,4}$ Evan Edmond, ${ }^{2}$ William T. Clarke, ${ }^{2,4}$ Emir Uzay, ${ }^{5}$ Jonathan P. Farthing, ${ }^{1, *}$ and Charlotte J. Stagg ${ }^{2, *}$

${ }^{I}$ College of Kinesiology, University of Saskatchewan, Canada.

${ }^{2}$ Wellcome Centre for Integrative Neuroimaging, FMRIB, Nuffield Department of Clinical Neurosciences, University of Oxford, United Kingdom.

${ }^{3}$ Section on Functional Imaging Methods, National Institutes of Mental Health, National Institutes of Health, Bethesda, Maryland, United States.

${ }^{4}$ Department of Clinical and Movement Neurosciences, University College London, United Kingdom. ${ }^{5}$ School of Health Sciences, College of Health and Human Sciences, Purdue University, United States.

${ }^{*}$ Co-senior authors.

In humans, motor learning is underpinned by changes in functional connectivity (FC) across the sensorimotor network. Unilateral exercise-induced fatigue increases FC in the ipsilateral primary motor cortex (M1) and supplementary motor area (SMA), which are involved in motor planning and execution of the contralateral hand, making unilateral fatiguing exercise a potential method for augmenting contralateral motor performance. In a within-subjects randomized cross-over design, 15 right-handed adults had two magnetic resonance imaging (MRI) sessions, where functional MRI and MR Spectroscopic Imaging were acquired before and after repeated right-hand contractions at either $5 \%$ or $50 \%$ maximum voluntary contraction (MVC). Before and after scanning, response times (RTs) were determined in both hands to quantify motor performance. The purpose was to investigate the effects of unimanual handgrip fatigue on M1 and SMA FC, neurochemical concentrations and motor performance of the contralateral non-fatigued hand. Unimanual motor fatigue was predicted increase motor performance in the non-fatigued hand and decrease interhemispheric homologous FC of M1 and SMA, via an altered excitatory/inhibitory state within these cortical motor regions by increasing glutamate and/or decreasing gamma-aminobutyric acid (GABA). Nine-minutes of $50 \%$ MVC contractions resulted in fatigue in the right-hand $(50 \%: \beta=-0.912 \pm 1.070 ; 5 \%$ : $\beta=-0.022 \pm 0.107 ; p=0.006, g=0.801$ ). This unimanual fatigue improved motor performance, as indexed by decreased RTs in the left-hand for the 50\% MVC condition only (paired $t$-test for left-hand, $p=0.037$, $\mathrm{g}=0.574$ ). These behavioural effects were supported by a significant ROI $\times$ time interaction in the $50 \%$ MVC condition where an increase in SMA-SMA and decrease in M1-M1 interhemispheric FC was observed $\left(\Delta\right.$ Z-scores for M1-M1: -0.132 ; SMA-SMA: 0.199; $p=0.004, \eta_{p}{ }^{2}=0.461$ ). Finally, the improvements in contralateral motor performance were correlated with the degree of fatigue-induced decrease in GABA in left
M1 $(r=0.515, p=0.048)$ and right SMA $(r=-0.518, p=0.047)$. Taken together, these results support the hypothesis that unilateral fatiguing exercise enhances motor performance in the contralateral hand with healthy participants and detail the neural and neurochemical changes that support the behavioural effects. These findings may be a useful therapeutic intervention in a range of neurological and orthopaedic conditions.

\section{Similar rate of development of the $\dot{\mathrm{VO}}_{2}$ slow component and peripheral fatigue across different exercise durations and intensities near the MLSS}

Rafael de Almeida Azevedo, ${ }^{1}$ Daniel A. Keir, ${ }^{3,4}$ Jonas Forot, ${ }^{1,2}$ Danilo Iannetta, ${ }^{1}$ Guillaume Y. Millet, ${ }^{1,5,6}$ and Juan M. Murias ${ }^{1}$

${ }^{I}$ Faculty of Kinesiology, University of Calgary, Calgary, Canada.

${ }^{2}$ University of Grenoble Alpes, Grenoble, France.

${ }^{3}$ School of Kinesiology, The University of Western Ontario, London, Canada.

${ }^{4}$ Department of Medicine, Toronto General Hospital Research Institute, University Health Network, Toronto, Canada.

${ }^{5}$ Univ Lyon, UJM-Saint-Etienne, Inter-university Laboratory of Human Movement Biology, EA 7424 F-42023, Saint-Etienne, France.

${ }^{6}$ Institut Universitaire de France (IUF).

The oxygen uptake slow component $\left(\dot{\mathrm{VO}}_{2 \mathrm{SC}}\right)$ and peripheral muscle fatigue have been proposed to be connected. If this idea is true, then changes in these variables should remain coupled within a single bout of exercise in a time- and exercise intensity-dependent manner. Sixteen participants ( 7 females) visited the laboratory on seven separate occasions, as follows: 1) three bouts of 6 min of baseline cycling at $20 \mathrm{~W}$, followed by 6 min of moderate-intensity cycling, proceeded by a ramp incremental test; 2-3) 30-min constant power output (PO) exercise bouts to determine the maximal lactate steady state (MLSS); 4-7) constant-PO exercise bouts to task failure (TTF) in pseudorandomized order, at POs corresponding to: (i) $15 \%$ below MLSS; (ii) $10 \mathrm{~W}$ below MLSS; (iii) MLSS; and (iv) $10 \mathrm{~W}$ above MLSS, wherein the later intensity was always the first and the remaining were performed in randomized order. The $\mathrm{V}_{2 S C}$ during the constant-PO bouts was characterized based on each individual $\mathrm{V}_{2}$ kinetics during moderate transitions. Pulmonary oxygen uptake $\left(\mathrm{VO}_{2}\right)$ was continuously measured during the constant-PO bouts. Neuromuscular fatigue was characterized by isometric maximal voluntary contractions and femoral nerve electrical stimulation of knee extensors to measured voluntary activation and peripheral fatigue at baseline, at $\min 5,10,20,30$, and at TTF of the constant-PO bouts. The development of the $\mathrm{VO}_{2 S C}$ and peripheral fatigue were strongly correlated across time ( $r^{2}$ adj range of 0.64-0.80) and amongst each exercise intensity $\left(r^{2}\right.$ adj range of $\left.0.26-0.30\right)$ (all $\left.p<0.001\right)$. The present study demonstrated that the development of the $\mathrm{VO}_{2 S C}$ and peripheral fatigue were similar in a time- and intensity-dependent manner. It is suggested that the reduced muscle contractile capacity might affect both variables, even though the source of its reduction is currently uncertain. (RAA supported by Eyes High Doctoral Recruitment Scholarship; Dr. Juan M. Murias' work is supported by the Natural Sciences and Engineering Research Council of Canada (RGPIN-2016-03698) and the Heart \& Stroke Foundation of Canada (1047725).)

\section{Ketone gavage alters BACE1 content and activity in a brain region specific manner}

B.J. Baranowski, ${ }^{1}$ A. Mohammad, ${ }^{1}$ H. Shamshoom, ${ }^{2}$ D.C. Wright, ${ }^{2}$ J.J. Walsh, ${ }^{3}$ and R.E.K. MacPherson ${ }^{1}$

${ }^{1}$ Health Sciences, Brock University, St. Catharines, ON L2S 3A1, Canada.

${ }^{2}$ Human Health and Nutritional Sciences, University of Guelph, Guelph, ON N1G 2W1, Canada

${ }^{3}$ Department of Kinesiology, McMaster University, Hamilton, ON L8S 4K1, Canada

${ }^{4}$ Centre for Neuroscience, Brock University, St. Catharines, ON L2S 3A1, Canada.

Brain-derived neurotrophic factor (BDNF) content declines with age and is reduced in patients with Alzheimer's disease. Cell culture and 
animal studies indicate that the ketone body $\beta$-hydroxybutyrate $(\beta \mathrm{HB})$ can reduce beta-amyloid plaques, however the mechanisms are unknown. Research has demonstrated that $\beta \mathrm{HB}$ promotes the expression of BDNF in the hippocampus of mice and evidence from human studies suggests ketone-induced cognitive improvements. BDNF treatment has been shown to reduce the activity of BACE1, the rate limiting enzyme in the amyloidogenic cascade. We aimed to determine the effect of $\beta$ HB supplementation on brain BDNF content/signaling and the subsequent effects on cognitive function and amyloidogenic processing. Male C57BL6 mice were be assigned into 1) Saline $(\mathrm{n}=20)$ and 2) $\beta \mathrm{HB}(\mathrm{n}=20)$. $\beta \mathrm{HB}$ mice underwent oral gavage of $\beta \mathrm{HB}(3 \mathrm{mg} / \mathrm{g}$ b.w. of $\beta \mathrm{HB})$. To examine the effects of acute $\beta \mathrm{HB}$ supplementation on cognition mice underwent object recognition training prior to the gavage and were tested 4 - and 24-hours post-gavage ( $\mathrm{n}=10$ /group). Post-euthanasia, prefrontal cortex and hippocampal samples were dissected to examine BDNF signaling and the amyloidogenic pathway. Oral gavage of $\beta \mathrm{HB}$ elevated circulating $\beta \mathrm{HB}$ content, reduced plasma glucose, and resulted in higher skeletal muscle glycogen content compared to saline. $\beta \mathrm{HB}$ treatment resulted in a trend towards higher exploration time of the novel object (main effect of $\beta \mathrm{HB} p=0.07$ ), potentially indicative of enhanced cognitive performance. In the prefrontal cortex, there was lower BACE1 activity at the 24-hour time point. However, the hippocampus had higher BACE1 activity at the same time point. This study demonstrates for the first time that acute $\beta \mathrm{HB}$ administration in mice modulates BACE1 activity in a time and brain region dependent manner. These preliminary findings support the potential of ketones as a future therapeutic supplement for neurodegenerative diseases. Further work will explore the mechanistic link between $\beta \mathrm{HB}$ and BACE1 regulation. (Funded by a Brock University Explore Grant)

\section{Effects of high-intensity interval exercise on natural killer cell number and function in pre- and post-pubertal children}

\section{G. Barber, ${ }^{*}$ E. Hauck, ${ }^{*}$ I. Ushcatz, M. Bjelica, and J. Obeid \\ Child Health and Exercise Medicine Program, McMaster University, Hamilton, ON, Canada \\ *Authors contributed equally to this work.}

Natural killer (NK) cells are lymphocytes that can identify and destroy infected or cancerous cells without prior sensitization. Acute exercise increases circulating NK cell numbers and function in adults, but the effects of acute exercise in children are less clear. Therefore, this study examined the effects of a bout of high-intensity interval exercise on NK cell recruitment and cytotoxicity in pre- vs. post-pubertal children. Healthy pre- $(\mathrm{N}=5,2 \mathrm{~F}$; mean $\pm \mathrm{SD}$, age: $10.0 \pm$ 0.9 years) and post-pubertal ( $\mathrm{N}=6,3 \mathrm{~F}$; age: $17.2 \pm 1.0$ years) children completed an aerobic fitness test to determine ventilatory threshold (VT). At a second visit, participants completed high-intensity interval cycling (20 x 15-sec all-out cycling at $25 \%$ above VT). Blood samples were collected at rest (PRE), immediately after exercise (POST), and after 30-minutes (REC1) and 60-minutes (REC2) recovery. NK cells as a percentage of lymphocytes (\%NK) and NK cytotoxic activity (specific lysis and NKCA per cell) against a cancer cell line (K562) were quantified using flow cytometry. \%NK increased from PRE $(7.2 \pm 3.1 \%)$ to POST (15.5 $\pm 8.1 \%)$ and returned to baseline by REC1 $(6.0 \pm 2.8 \%) ; \mathrm{p}<0.001$. At POST, \%NK were higher in post-pubertal $(20.9 \pm 6.2 \%)$ compared with pre-pubertal children ( $9.1 \pm 4.3 \%$; $\mathrm{p}<0.001)$. Specific lysis was not different between PRE $(26.8 \pm 5.8 \%)$ and POST $(31.8 \pm 10.9 \%)$ but was higher at POST compared with REC1 $(23.6 \pm 6.3 \%)$ and REC2 (19.4 $\pm 5.2 \%$; both $\mathrm{p}<0.005)$. Specific lysis at REC2 was lower than PRE $(\mathrm{p}<0.05)$. NKCA per cell increased from PRE $(0.58 \pm 0.50)$ to REC2 $(1.5 \pm 1.3 ; \mathrm{p}<0.005)$ and from POST $(0.28 \pm 0.25)$ to REC1 $(1.3 \pm 1.6$; $\mathrm{p}<0.05)$ and REC2. The effects of exercise on NK function were not different between pubertal groups. Our findings suggest that in children, exercise induces changes in NK recruitment and function along different timelines. Future research will explore the effects of exercise training on NK cells in healthy and immunocompromised pediatric populations. This study was funded by an NSERC Discovery Grant.

\section{Moderate altitude reduces the power output at the maximal lactate steady state}

A.T. Beever, A.Y. Zhuang, J.M. Murias, S.J. Aboodarda, and M.J. MacInnis

Faculty of Kinesiology, University of Calgary, Calgary, AB T2N IN4, Canada.

The maximal lactate steady state (MLSS) identifies the upper limit of sustainable exercise; however, the extent to which an individual's MLSS is affected by changes in altitude is unknown. The purpose of this study was to determine the impact of three different simulated altitudes on the MLSS power output (MLSS ${ }_{\mathrm{p}}$ ) as well as physiological responses to exercise at and $10 \mathrm{~W}$ above the MLSS $_{\mathrm{p}}\left(\right.$ MLSS $\left._{\mathrm{p}+10}\right)$. Eleven (4 females, 7 males) mild-altitude residents (mean[SD]: 28[4] years, $54.3[6.9] \mathrm{mL} \cdot \mathrm{kg}^{-1} \cdot \mathrm{min}^{-1}$ ) performed 30-min constant-load cycling tests at MLSS $\mathrm{p}_{\mathrm{p}}$ and $\mathrm{MLSS}_{\mathrm{p}+10}$ in simulated sea-level (“SL”; $0 \mathrm{~m}$ ), mild ("MILD”; $1111 \mathrm{~m}$ ) and moderate ("MOD"; $2222 \mathrm{~m}$ ) altitudes. Cardiorespiratory measures were recorded continuously throughout each cycling bout, while capillary blood samples and perceptual responses were collected every five min. Neuromuscular fatigue was determined pre- and post-exercise using femoral nerve electrical stimulation. The MLSS $\mathrm{p}$ and the associated oxygen consumption $\left(\mathrm{VO}_{2}\right)$ were lower in MOD $\left(209[54] \mathrm{W} ; 40.4[5.6] \mathrm{mL} \cdot \mathrm{kg}^{-1} \cdot \mathrm{min}^{-1}\right)$ compared to SL (230[56] W, $\left.\mathrm{p}<0.001 ; 45.0[8.5] \mathrm{mL} \cdot \mathrm{kg}^{-1} \cdot \mathrm{min}^{-1}, \mathrm{p}=0.005\right)$ and MILD (225[58] W, $\mathrm{p}<0.001 ; 42.7[6.5] \mathrm{mL} \cdot \mathrm{kg}^{-1} \cdot \mathrm{min}^{-1}, \mathrm{p}=0.009$ ), but were not different between MILD and SL $(\mathrm{p}>0.05)$. Blood lactate concentration, ventilation, heart rate and perceptual responses to exercise did not differ across altitudes ( $\mathrm{p}>0.05)$, though they were exacerbated at MLSS $_{\mathrm{p}+10}$ compared to MLSS $\mathrm{p}$ within each condition $(\mathrm{p}<0.05)$. Neuromuscular fatigue was evident by decreased quadriceps isometric maximal voluntary contraction (MLSS : 675[137] to 585[136] N; $\mathrm{p}<0.05$ ), low-frequency doublet (MLSS ${ }_{\mathrm{p}}$ : 260[56] to $184[47] \mathrm{N}$; $\mathrm{p}<0.05$ ), and single twitch force $\left(\right.$ MLSS $_{\mathrm{p}}$ : $177[38]$ to $145[42] \mathrm{N} ; \mathrm{p}<0.05$ ) (rates of decline were similar for MLSS $_{\mathrm{p}+10}$ ), but there were no differences between conditions at $\operatorname{MLSS}_{\mathrm{p}}(\mathrm{p}>0.05)$ or $\mathrm{MLSS}_{\mathrm{p}+10}(\mathrm{p}>0.05)$. In summary, the

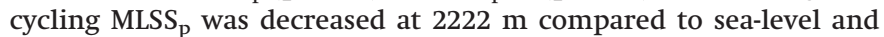
$1111 \mathrm{~m}$, but metabolic, cardiorespiratory, perceptual, and neuromuscular responses to exercise were similar across altitudes at the same relative exercise intensities. (Funded by the Natural Sciences and Engineering Research Council of Canada)

\section{Sex differences in skeletal muscle adaptations of middle-aged} adults after 10 weeks of progressive resistance training

E. Binet, ${ }^{1}$ N. Collao, ${ }^{1}$ C. McKenna, ${ }^{2}$ A. Salvador, ${ }^{3}$ N.A. Khan, ${ }^{2,3}$ S. Paluska, ${ }^{4}$ N. Burd, ${ }^{2,3}$ and M. De Lisio ${ }^{1,5}$

${ }^{I}$ School of Human Kinetics, University of Ottawa, Ottawa, ON K1H $8 \mathrm{Ll}$

${ }^{2}$ Division of Nutritional Sciences, University of Illinois at Urbana-Champaign, Urbana, IL 61801 ${ }^{3}$ Kinesiology and Community Health, University of Illinois at Urbana-Champaign, Urbana, IL 61801.

${ }^{4}$ Family Medicine, University of Illinois at Urbana-Champaign, Urbana, IL 61801.

${ }^{5}$ Department of Cellular and Molecular Medicine, University of Ottawa, Ottawa, ON KIN $8 \mathrm{L1}$.

Resistance training induces myofibre hypertrophy and is an effective training method to increase strength. It has been shown that both men and women experience myofibre hypertrophy but there are conflicting findings regarding the degree to which the sexes adapt. It is unclear if men and women respond similarly to resistance training with protein supplementation, specifically at the myofibre level. To address this gap, middle-aged adults, 40-64 years, participated in a 10-week progressive, whole-body resistance training intervention coupled with protein supplementation and constant dietary counselling to ensure adequate protein consumption. Body weight, body mass index, lean body mass, and leg press strength all significantly increased from pre to post resistance training in both sexes. There were no significant sex differences in protein consumption; however, 
protein consumption significantly increased from pre to during resistance training. Males had significantly larger myofibre cross-sectional area, due to significantly larger type II fibres than females, with no effect of resistance training. Males had significantly more myonuclei per type II fibre, and resistance training significantly reduced the number of myonuclei per type I fibres. Males had significantly higher myonuclear domain size than females in type II fibres with no effect of resistance training. There were no significant sex or time effects on the proportion of type I or II fibres. Sex differences were detected in type II fibre morphology; however, resistance training with sufficient protein consumption had minimal effects. These results further clarify sex-based differences in muscle morphology at the myofibre level in response to resistance training in middle-aged adults. (Funded by the Beef Checkoff.)

\section{Consumption of a high-fat-high-sucrose diet partly prevents cardiac mechanical adaptations to aerobic and resistance exercise training}

\author{
K.R. Boldt, ${ }^{1,2}$ V. Joumaa, ${ }^{1}$ S. Mattiello, ${ }^{3}$ and W. Herzog ${ }^{1}$ \\ ${ }^{1}$ Human Performance Laboratory, Faculty of Kinesiology, University of Calgary, Calgary, Canada. \\ ${ }^{2}$ Trent University, Peterborough, Ontario, Canada. \\ ${ }^{3}$ Department of Physical Therapy, Center of Biological and Health Sciences, Federal University of \\ São Carlos, São Carlos, Brazil.
}

Resistance training has been shown to exert a protective effect on the health of the heart. This protective effect is thought to be, in part, due to mechanical adaptations of the myocardium. Conversely, consumption of a high-fat-high-sucrose (HFHS) diet impairs myocardial contractile function. However, it is not clear whether a HFHS prevents the mechanical adaptations of the myocardium to resistance training. The purpose of this study was to evaluate the effects of consuming a high-fat-high-sucrose diet on cardiac mechanical adaptations to resistance training. Threemonth-old Sprague-Dawley rats were randomized into three groups: 1) chow-fed sedentary animals $(n=5), 2)$ chow-fed resistance-trained animals $(\mathrm{n}=6)$, and 3) HFHS-fed resistance-trained animals $(\mathrm{n}=7)$. The resistance exercise training consisted of weighted ladder climbing three days per week for 12 weeks. The chow diet consisted of $25 \%$ protein, $5 \%$ fat, and $48 \%$ carbohydrates (3\% carbohydrates from sucrose), and $22 \%$ fiber and moisture. The HFHS diet consisted of $20 \%$ protein, $20 \%$ fat, and $50 \%$ carbohydrates (>97\% carbohydrates from sucrose), and $10 \%$ fiber and moisture. Following 12 weeks, hearts were dissected, and chemically skinned cardiac muscle bundles were tested for their maximal isometric active force, unloaded shortening velocity, and calcium sensitivity. Maximal isometric stress (force normalized to cross-sectional area) was determined by transferring the sample to a maximal activating solution. Unloaded shortening velocity was determined using the slack test (rapidly shortening the sample, causing it to go slack, and measuring the time until tension redevelops). Calcium sensitivity was determined by transferring the sample through solutions of increasing calcium concentration and recording force output. Fibre bundles from chow-fed resistance-trained animals had greater maximal isometric stress ( $34.2 \pm 4.2$ vs $23.7 \pm$ $\left.4.9 \mathrm{mN} / \mathrm{mm}^{2}\right)$, shortening velocities $(1.26 \pm 0.04$ vs $1.15 \pm 0.05$ bundle length/s), and calcium sensitivities $\left(\mathrm{pCa}_{50} 6.07 \pm 0.21\right.$ vs $5.78 \pm 0.17$ ) than those of chow-fed sedentary controls. Bundles from the HFHS resistance-trained animals had greater maximal isometric stress $\left(33.3 \pm 11.9 \mathrm{mN} / \mathrm{mm}^{2}\right)$ but no change in unloaded shortening velocity (1.16 \pm 0.03 bundle length/s) or calcium sensitivity $\left(\mathrm{pCa}_{50} 5.75 \pm\right.$ 0.13) compared to those of the chow-fed sedentary animals. Thus, resistance training results in mechanical adaptations of the myocardium, which are partly prevented when performed in conjunction with a HFHS diet.
The effects of an acute bout of high-intensity interval exercise in the morning vs. evening on the magnitude of the morning blood pressure surge

\section{J. Bommarito, M. Nardone, J.F. Burr, and P.J. Millar}

Human Health and Nutritional Sciences, University of Guelph, Guelph, ON NIG 2W6, Canada.

The morning blood pressure surge (MBPS) is an essential anticipatory event to maintain adequate blood flow during increased activity in the morning. However, an exaggerated MBPS is a strong predictor of cardiovascular events and all-cause mortality. Following exercise, a prolonged decrease in blood pressure (BP) can occur, termed post-exercise hypotension ( $\mathrm{PEH}$ ). High-intensity interval exercise (HIIE) is characterized as recurring bursts of high-intensity exercise, interspersed with low-intensity, recovery exercise. HIIE produces superior PEH responses compared to moderate-intensity continuous exercise. The time exercise is performed also modulates the PEH response. Thus, the present study aims to assess the effects of an acute bout of HIIE performed in the morning vs. evening on the magnitude of the MBPS. In a randomized crossover trial, healthy young males $(\mathrm{n}=4)$ performed morning HIIE (between 0800-1000 hours), evening HIIE (between 1700-1900 hours), or no exercise (control) on three separate visits. One-minute bursts of high-intensity exercise were performed at $80 \%$ peak power and were interspersed with 1-minute recovery exercise performed at $15 \%$ peak power. Following each visit, participants were equipped with an ambulatory blood pressure monitor programmed to take measurement every $30 \mathrm{~min}$ for 24-hours. The MBPS was assessed with three calculations (sleep trough surge; STS, sleep trough morning surge; STMS, and prewaking surge; PS). No difference for the STS (Control $=16.5 \pm 12.4$; Morning $=11.5 \pm 3.7$; Evening $=22.5 \pm$ 13.3; $\mathrm{p}=0.22$ ), STMS (Control $=25.5 \pm 16.1$; Morning $=17.8 \pm 4.3$; Evening $=28.5 \pm 14.5 ; \mathrm{p}=0.32$ ), and PS (Control $=12.8 \pm 11.8$; Morning $=$ $6.8 \pm 6.8$; Evening $=15 \pm 8.1 ; \mathrm{p}=0.40$ ) has been observed. These preliminary findings suggest that HIIE performed in the morning or evening may not attenuate the morning blood pressure surge in young healthy men.

Impact of high intensity interval training with blood flow restriction on aerobic and anaerobic performances in endurance-trained athletes: preliminary results

\section{H. Bourgeois, P. Paradis-Deschênes, and F. Billaut}

Département de kinésiologie, Université Laval, Québec, QC, Canada

Although blood flow restriction (BFR) training has gained popularity amongst athletes and researchers, there is few evidence of the impact of high-intensity interval training (HIIT) combined with BFR on performance. This study investigated the impact of high-intensity interval training with blood flow restriction (BFR) on aerobic and anaerobic performance. 15 endurance-trained men (VO2 max: $66.53 \pm$ 7.55 [mean \pm SD]) included three sessions per week over three weeks of HIIT with (BFR, $n=9$ ) or without (CTL, $n=6$ ) cuffs inflated to $50-70 \%$ of their arterial occlusion pressure into their usual training. Before and after training, athletes completed a maximal incremental step test on a bike attached to a Smart trainer, a Wingate anaerobic test (WAnT) and a $5-\mathrm{km}$ cycling time trial on two different days. VO2max and maximal aerobic power (MAP) were assessed during the step test and power output was measured during all tests. Cohen's effect sizes (ES) were used to compare differences between groups. Compared to CTL, BFR possibly had a small effect on MAP (BFR: $5.12 \%$ vs CTL: $1.14 \%$, ES 0.23), anaerobic capacity (BFR: $3.03 \%$ vs CTL: $-0.28 \%$, ES 0.28 ) and total work at the WAnt (BFR: $3.14 \%$ vs CTL: $-0.14 \%$, ES 0.21), but not on VंO2max (BFR: $1.30 \%$ vs CTL: $2.77 \%$ ES -0.15 ), anaerobic power (BFR: $-2.43 \%$ vs CTL: $-3.76 \%$, ES 0.19 ) and $5-\mathrm{km}$ mean power-output (BFR: $1.72 \%$ vs CTL: $5.85 \%$, ES -0.21 ). These preliminary results suggests that HIIT with BFR could slightly improve aerobic and anaerobic capacity over a 
three-weeks period in endurance-trained athletes, but those improvements may not lead to performance gains in a short aerobic cycling effort.

\section{A multimodal intervention to improve brain function in older adults with mild cognitive impairment. Results from SYNERGIC}

N.W. Bray, ${ }^{1}$ F. Pieruccini-Faria, ${ }^{1}$ Q.J. Almeida, ${ }^{2}$ L. Bherer, ${ }^{3}$ T. Liu-Ambrose, ${ }^{4}$ L. Middleton, ${ }^{5}$ and M. Montero-Odasso ${ }^{1}$

${ }^{I}$ School of Kinesiology, University of Western Ontario, London, ON, Canada.

${ }^{2}$ Department of Kinesiology, Wilfrid Laurier University, Waterloo, ON, Canada.

Department of Medicine, Universitéde Montréal, Montreal, OC, Canada.

${ }^{4}$ Department of Physical Therapy, The University of British Columbia, Vancouver, BC, Canada.

${ }^{5}$ Department of Kinesiology, University of Waterloo, Waterloo, ON, Canada.

Prevention through lifestyle modification (i.e., physical exercise, cognitive training, nutrition, etc.) is considered the best "medicine" for maintaining brain function in old age. Mild cognitive impairment (MCI) represents a transitional state between "normal cognitive aging" and dementia (i.e., Alzheimer's disease). Functional connectivity (FC) refers to brain areas that are anatomically separate but temporally similar in their activation; it enables the completion of complex cognitive and physical functions. Altered FC represents an early marker in MCI. No study has assessed the impact of aerobic and resistance training with and without other lifestyle interventional strategies on FC in those with MCI. Our double-blind, 20-week randomized controlled trial evaluated the effect of aerobic and resistance training (combined exercise arm) with and without cognitive training and/or vitamin D supplementation (multimodal intervention arm) in those with MCI. 120 participants underwent 3-Tesla resting-state brain scans and a battery of physical and cognitive performance testing pre- and post-intervention. We assessed FC using the CONN Toolbox. Combined exercise improved (post-pre) FC of the hippocampus to the angular gyrus. The multimodal interventions did not produce any additional changes. Pearson correlation of change scores (post-pre) demonstrated moderate to large effect sizes between FC and physical (i.e., gait speed: $\mathrm{r}(30)=-0.365, \mathrm{p}=0.04)$, but not cognitive performance. However, no correlations survived correction for multiple comparisons. Combined exercise improved FC in regions implicated early in MCI. Adding cognitive training or vitamin D had no additional impact. Implications for behavioral outcomes require further investigation, but aerobic and resistance training alone may be sufficient to reverse some of the earliest brain changes observed in MCI, potentially delaying progression to Alzheimer's disease.

Factors affecting walking compliance in patients with peripheral artery disease

I.K.M. Brenner, ${ }^{1}$ C.A. Brown, ${ }^{2}$ S.M.J. Hains, ${ }^{2}$ J. Tranmer, ${ }^{2}$ D. Zelt, ${ }^{3}$ and P. Brown ${ }^{3}$

${ }^{I}$ Trent/Fleming School of Nursing, Trent University, Peterborough, ON K9L 0G2, Canada. ${ }^{2}$ School of Nursing, Queen's University, Kingston, ON K7L 3N6, Canada.

${ }^{3}$ Kingston Health Sciences Centre, Queen's University, Kingston, ON K7L 2V7, Canada.

The purpose of this study was to examine walking compliance in patients with peripheral artery disease. Forty-eight patients (aged $68 \pm 8$ years) with intermittent claudication (and/or and ankle brachial index $[\mathrm{ABI}]<0.9)$ were randomly assigned to either an exercise group ( $\mathrm{n}=27$, who performed a structured walking program 5 days per week over 12-weeks) or a comparison group ( $\mathrm{n}=21$, who performed usual daily activities). Members of both groups kept activity logs and were contacted biweekly by telephone. Data was obtained regarding patient demographics, medical history, physical function (treadmill walking and 6-min walk test [6MWT]), responses to the walking impairment (WIQ) and quality of life questionnaires (SF-36)). Eighty-one percent $(n=39)$ of the participants completed the study. Data was analyzed to determine factors (patient, therapy and disease-related) which contributed to adherence to the study as well as exercise compliance. Correlation and linear regression analyses were performed using gender, presence of co-morbidity, ABI, height, smoking, performance characteristics and responses to self-report questionnaires (the WIQ and SF-36) to determine predictors of exercise compliance. Patients, who were physically active prior to the study and who scored higher on their self-perceived walking ability (WIQ-distance score) as well as the physical function component of the SF-36 remained in the study. Prediction models indicated that gender, height, smoking status, SF-36 score and physical function (absolute claudication time and $6 \mathrm{MWT}$ ) were significant variables influencing exercise compliance. An understanding of these factors is helpful for those individuals prescribing exercise programs to patients with peripheral artery disease. (Funding for this study was provided by Queen's University, School of Nursing Freda Paltiel Award and a Southern Ontario Academic Health Sciences Innovation Grant.)

Differential postprandial amino acid responses following the consumption of isonitrogenous doses of Greek Yogurt and Skim Milk

J.K. Brown, ${ }^{1}$ C.G.R. Perry, ${ }^{1}$ T. Prior, ${ }^{2}$ S.M. Phillips,${ }^{2}$ L.E. Skelly, ${ }^{1}$ and A.R. Josse ${ }^{1}$

${ }^{I}$ School of Kinesiology and Health Science, York University, Toronto, ON, M3J 1P3, Canada. ${ }^{2}$ Department of Kinesiology, McMaster University, Hamilton, ON, L8S 4L8, Canada.

Upon consumption, dietary protein is digested into its constituent amino acids (AA) and absorbed into the blood stream before becoming bioavailable for tissue utilization. Recently, we demonstrated greater training-induced adaptations in muscular strength, muscle size and lean mass after Greek Yogurt (GY) consumption vs. carbohydrate pudding following 12 weeks of resistance training in young males. Because these training adaptations were also demonstrated with post-exercise milk consumption, and that AA digestion/absorption can impact muscle protein synthesis, we aimed to assess the rested postprandial AA response following the consumption of GY vs. skim milk (M). Ten healthy, recreationally active males (age 23.1 $\pm 1.1 y$; BMI $25.6 \pm 2.4 \mathrm{~kg} / \mathrm{m}^{2}$ ) participated in an acute, randomized, crossover protocol involving 2 conditions separated by a 1-week washout. GY and M (20g-protein each), were ingested at two feeding timepoints (baseline and 60minutes). Venous blood samples were taken at baseline, 30, 60, 90, 120, 150, 180 and 240 minutes. Plasma was analyzed for leucine and branched-chain amino acids (BCAA) maximal concentrations $\left(C_{\max }\right)$, time to maximal concentration $\left(\mathrm{T}_{\max }\right)$ and total area under the curve (AUC). Interactions (time $\mathrm{x}$ condition $\mathrm{P}=0.008$ ) were observed for both leucine and BCAAs such that leucine was higher after GY ingestion compared to $\mathrm{M}$ at 60- and 120-minutes and for BCAAs at 60-, 90- and 180-minutes $(\mathrm{P}<0.007)$. GY exceeded $\mathrm{M}$ for peak leucine (372 vs. $255 \mu \mathrm{mol} \cdot \mathrm{L}^{-1}$ ) and BCAA (1509 vs. $\left.800 \mu \mathrm{mol} \cdot \mathrm{L}^{-1}\right)$ concentrations $\left(\mathrm{C}_{\max } ; \mathrm{P}=0.004\right)$. $\mathrm{T}_{\max }$ for leucine and BCAAs was less for M vs. GY (30 vs. $90 \mathrm{~min}$, respectively; $\mathrm{P}=0.008)$. The $\mathrm{AUCs}$ for leucine and BCAAs were greater for GY vs. $\mathrm{M}(\mathrm{P}=0.004)$. Our findings demonstrate that GY elicits a greater blood leucine/BCAA response compared to an isonitrogenous bolus of $\mathrm{M}$ over 4 hours. These findings further exemplify dairy as a functional anabolic food, and the utility of GY, as a post-exercise alternative to milk.

The effects of high intensity interval training on PTSD symptomology

J.K. Bryan, T. Black, L.A. Stuart-Hill, and C.A. Gaul

Exercise and Physical Health Education, University of Victoria, Victoria, BC V8W 2Y2, Canada.

The purpose of this study was to determine the effect of high intensity interval training (HIIT) on post traumatic stress disorder (PTSD) 
and to compare the results of HIIT to a short, deep breathing session (BRTH) that is commonly used to manage PTSD symptoms. Previous research has demonstrated a positive effect of exercise on PTSD and related mental health concerns. Seven volunteers (six females and one male) participated in this study. All participants completed two groups of sessions, one comprised of three HIIT sessions and one of three BRTH sessions. Each group of sessions took place over approximately ten days, with a 4 -week washout period between the two protocols. The HIIT sessions each involved eight sets of a work rest ratio of 20 -second exercise to 10 -second rest, with a 60-second break between the fourth and the fifth set for a total of 5 minutes. The BRTH sessions involved the use of an established 5-minute deep breathing PTSD therapy protocol. PTSD symptoms were measured using the PTSD Checklist (PCL). PCL measures were taken before (pre-test) and after (post-test) the intervention. Within the HIIT intervention, mean post-test Total PCL scores were significantly reduced compared to pre-test scores $(\mathrm{p}<0.005)$, while BRTH scores did not significantly change. Two subscales in the PCL, Intrusion and Avoidance were also significantly reduced $(\mathrm{p}<0.0005)$ following HIIT but not BRTH. All other subscales scores were lower following both HIIT and BRTH, though were not statistically significant different from pre-test values. No statistical between-intervention differences were detected. The preliminary results from this research provide evidence that acute use of HIIT exercise provides similar or better positive effects on PTSD symptoms as BRTH and that HIIT exercise can be used as a symptom management tool for PTSD.

\section{The Comparison of Muscle Function Loss of Respiratory Muscles} and the Tibialis Anterior

J.S. Chan, L.M. Mann, C.J. Doherty, B. Thompson, S.A. Angus, M. Devries-Aboud, R.L. Hughson, and P.B. Dominelli

Department of Kinesiology, University of Waterloo, Waterloo, ON, N2L 3G1, Canada.

The respiratory muscles (RM) are crucial in maintaining ventilation; however, may atrophy faster than other skeletal muscles when unloaded. Respiratory muscle training (RMT) improves RM strength and attenuates the RM metaboreflex; however, the time course after detraining is less known. We sought to determine the time course of change in RM strength and the metaboreflex after five weeks of RM detraining. An experimental group (2F, 5M; 26士 3.7yrs) completed 5 weeks of RMT and tibialis anterior (TA) training (5 days/week at 50\% maximal inspiratory pressure (MIP) and $50 \%$ maximal isometric force, respectively) followed by 5 weeks of no training (detraining) while a control group (1F, 2M; 24 1 yrs) underwent no intervention. During pre-training, post-training, and post-detraining, all participants underwent a resistive breathing task (RBT) to failure (breathing frequency: $15 \mathrm{bpm}$, at 60\% MIP, duty cycle 50:50) while heart rate (HR) and mean arterial blood pressure (MAP) were measured. The control group had a minimal increase in MIP and TA strength $\left(105 \pm 41\right.$ vs $108 \pm 40$ vs $114 \pm 38 \mathrm{cmH}_{2} \mathrm{O} ; 87 \pm 15$ vs $88 \pm 9$ vs $94 \pm 5 \mathrm{~N}$ for pre-training, post-training, and post-detraining, respectively) and inconsistent changes in MAP (MAP ${ }_{\text {pre }}$ vs $\mathrm{MAP}_{\text {post }}$ vs $\mathrm{MAP}_{\text {post-detraining: }}+12 \pm 2.5$ vs $+9 \pm 6.1$ vs $+14 \pm 5.4 \%$ ). Five weeks of training increased $\mathrm{RM}(+20 \pm 7.5 \%, \mathrm{p}<0.05)$ and TA $(+29 \pm 15 \%, \mathrm{p}<0.05)$ strength and both remained elevated after 5 weeks of detraining

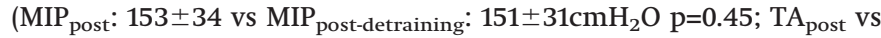

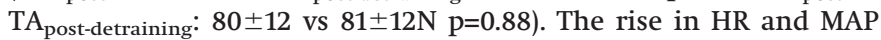
during RBT was attenuated post-training $\left(\mathrm{HR}_{\mathrm{pre}}:+40 \pm 20 \%\right.$ vs $\mathrm{HR}_{\text {post }}$ : $+26 \pm 14.5 \%$ bpm, $\mathrm{p}<0.05$; $\mathrm{MAP}_{\text {pre: }}:+23 \pm 8 \%$ vs $\mathrm{MAP}_{\text {post }}:+11 \pm 6.4 \% \mathrm{mmHg}$, $\mathrm{p}<0.05$ ) during the time matched, final minute of RBT. After detraining, the rise in HR and MAP during the RBT remained attenuated compared to pre-training $\left(\mathrm{HR}_{\text {pre }}:+40 \pm 20 \%\right.$ vs $\mathrm{HR}_{\text {post-detraining: }}$ :

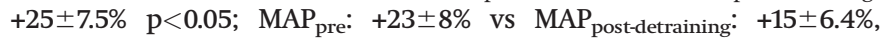
$p<0.05)$. In conclusion, RM and TA strength gains and the attenuation of the RM metaboreflex persist after 5 weeks of detraining. (Funding: NSERC)
Habitual cannabis users demonstrate altered cardiac mechanics at rest, but not during isometric handgrip exercise

C.P. Cheung, A.M. Coates, P.J. Millar, and J.F. Burr

Human Health and Nutritional Sciences, University of Guelph, Guelph, ON N1G 2WI, Canada.

Recreational cannabis use is highly prevalent, both in Canada and globally. Previous investigations have demonstrated that acute cannabis consumption increases resting heart rate and alters both left ventricular function and systolic time intervals; however, it is unknown whether habitual cannabis consumption exerts chronic effects on cardiac structure and function. Using a cross-sectional observational design, we explored whether habitual cannabis use is associated with cardiac impairments at rest, or during an exercise challenge. We performed 2-D transthoracic echocardiographic examinations at rest and during 3-minutes of isometric handgrip exercise (30\% maximal voluntary contraction) in individuals who smoked cannabis a minimum of once per week for three years $(\mathrm{n}=14)$ and non-using controls $(\mathrm{n}=14)$. Speckle tracking analysis was performed in a subset of cannabis users $(n=8)$ and controls $(n=8)$ to examine left ventricular mechanics. Groups were matched for age, sex, ethnicity, physical activity levels, and body size. Traditional measures of cardiac structure and left ventricular systolic and diastolic function were similar between groups, both at rest and during isometric handgrip exercise. However, speckle tracking analysis revealed that cannabis users demonstrated reduced left ventricular peak apical rotation (cannabis users: $5.5 \pm 3.8^{\circ}$, controls: $9.6 \pm 1.5^{\circ}$; $\mathrm{p}=0.02$ ), peak apical systolic rotation velocity (cannabis users: $45.2 \pm$ $20.7^{\circ} / \mathrm{s}$, controls: $71.8 \pm 15.5^{\circ} / \mathrm{s} ; \mathrm{p}=0.01$ ), peak apical diastolic rotation velocity (cannabis users: $-47.9 \pm 13.8^{\circ} / \mathrm{s}$, controls: $-68.5 \pm 19.0^{\circ} / \mathrm{s}$; $\mathrm{p}=0.01$ ), and increased peak longitudinal diastolic strain rate (cannabis users: $1.7 \pm 0.3 \% / \mathrm{s}$, controls: $1.3 \pm 0.4 \% / \mathrm{s} ; \mathrm{p}=0.02$ ) at rest compared to controls. No differences in left ventricular mechanics were observed between groups in response to isometric handgrip exercise. These findings suggest that in young, otherwise healthy individuals, habitual cannabis use does not impact traditional measures of cardiac structure, systolic function, diastolic function, or the cardiac response to isometric handgrip exercise; however, cannabis use may be associated with altered resting left ventricular mechanics.

\section{NHL Combine fitness test results predict long-term career performance: a longitudinal 25-year analysis}

J.N. Cohen, ${ }^{1}$ K.M.A. Thompson, ${ }^{2}$ V.K. Jamnik, ${ }^{2}$ N. Gledhill, ${ }^{2}$ and J.F. Burr ${ }^{1}$

${ }^{1}$ Human Health and Nutritional Sciences, University of Guelph, Guelph, ON NIG 2WI, Canada ${ }^{2}$ Kinesiology and Health Science, York University, Toronto, ON M3J 1P3, Canada.

Hockey players eligible for professional play are judged on junior performance, physical fitness and predicted potential by National Hockey League (NHL) teams. Combine fitness tests have been previously shown to predict draft order in the NHL, but not actual NHL career performance. Therefore, we examined relationships between NHL Combine fitness test results and long-term (5-10 seasons) NHL performance. NHL Combines (1994-2007) involved a standardized protocol, testing players' aerobic and anaerobic capacities, and both upper- and lower-body strength. Player performance (1995-2020) was quantified using publicly available statistics including: cumulative points, time-on-ice (TOI), and NHL career length. Forward $(\mathrm{n}=834)$ and defensive $(n=490)$ positions were analyzed separately to control for the differing positional expectations. Goalies were not considered in this analysis. 13 input variables collected at the NHL Combine were used to predict NHL performance outcomes by employing stepwise linear regression. The predictive capacity of all models employed ranged from $2-16 \%$ of variance explained. Peak leg power commonly predicted NHL career length when all players are considered (forwards: $\mathrm{R} 2=0.02$, defence: $\mathrm{R} 2=0.05$, both $\mathrm{P}<0.01$ ). However, amongst those players participating in at least 5 NHL seasons ( $<35 \%$ initial cohort), cumulative 
points and TOI were inversely predicted by upper-body push-strength variables in forwards and defence $(\mathrm{R} 2=0.03-0.14$, all $\mathrm{P}<0.01)$. Similarly, forwards and defensemen who participated in $10 \mathrm{NHL}$ seasons $(<20 \%$ initial cohort) displayed similar yet more robust inverse relationships between upper-body push-strength and both points and TOI ( $R 2=0.06-$ 0.16 , all $\mathrm{P}<0.01$ ). These data suggest peak leg power predicts NHL career length, whilst upper-body strength is inversely related to long-term (10 seasons) performance. NHL Combine fitness testing provides insight to a player's NHL career potential and should be used as a tool to inform decision-making and player development strategies.

\section{Late effects of radiation therapy on skeletal muscle fibro/ adipogenic progenitor and satellite cell crosstalk}

\author{
N. Collao, ${ }^{1}$ E. Gobel, ${ }^{2}$ D. D’Souza, ${ }^{1}$ J. Larkin, ${ }^{1}$ J. Lloyd, ${ }^{1}$ and M. De Lisio ${ }^{1,3}$ \\ ${ }^{1}$ Faculty of Health Science, School of Human Kinetics, University of Ottawa, ON KIN 6N5, Canada. \\ ${ }^{2}$ Faculty of Science, Department of Biology, University of Ottawa, ON KIN 6N5, Canada. \\ ${ }^{3}$ Faculty of Medicine, Department of Cellular and Molecular Medicine, Centre for Neuromuscular \\ Disease, University of Ottawa, ON KIN 6N5, Canada
}

Childhood cancer survivors are particularly susceptible to the longterm late effects of radiation therapy given their long-life span after therapy, high prevalence of radiation therapy for treating common childhood cancers, and their exposure during a time of rapid tissue development. Fibro/adipogenic progenitors (FAPs) are necessary for muscle maintenance and regeneration via paracrine regulation of muscle (satellite) stem cells (MuSCs). While radiation induces muscle atrophy, fibrosis, and depletes skeletal MuSCs, an understanding of the role of radiation therapy in regulating FAPMuSC crosstalk remains unknown. To address this gap; one lower limb of 5-6-week-old male CBA mice was exposed to a therapeutic dose of irradiation (IR); while the contralateral limb served as the non-IR control (CON). Mice were sacrificed at 3, 7, 14, and 56 days post-IR during postnatal development. Similar to clinical reports, radiation resulted in lower myofibre cross-sectional area, smaller myonuclear domain, and a higher proportion of smaller caliber myofibres (all $\mathrm{p} \leq 0.05$ vs. CON). Immunofluorescence and flow cytometry analyses revealed fewer MuSCs and FAPs in muscle postIR at 56 days (all $\mathrm{p} \leq 0.05$ vs. CON). To directly investigate the effects of radiation therapy on FAP paracrine factor secretion, condition media (CM) from FAPs isolated from skeletal muscle 56 days post-IR was applied to MuSCs. Irradiated FAP-CM impaired myoblast fusion and differentiation ( $\mathrm{p} \leq 0.05$ vs. CON). Similar findings were observed by immunofluorescence analysis of muscle biopsies from human patients showing a $50 \%$ reduction of FAPs after radiation therapy. These findings suggest that radiation alters the FAP secretome to impair myogenesis. Thus, restoring a healthy FAP secretome may prevent the long-term negative late effects of radiation therapy in childhood cancer survivors. (Funding provided by CONICYT-CHILE Doctoral Research Award and the Doctoral Fellowship for Advancement of Biological Perspectives for Exercise Interventions Across the Lifespan (NC) and NSERC (MD)).

\section{The history dependence of torque following eccentric exercise- induced muscle weakness}

\section{V.S. Contento, H.L. Jodoin, and G.A. Power}

Human Health and Nutritional Sciences, University of Guelph, Guelph, ON N1G 2W6, Canada.

Following active lengthening or shortening contractions, isometric steady-state torque is increased (residual torque enhancement; rTE) or decreased (residual torque depression; rTD), respectively, compared to purely isometric contractions at the same muscle length and level of activation. Though the mechanisms underlying the history dependence of force have been investigated extensively, little is known about the influence of exercise-induced muscle weakness on rTE and rTD. The purpose of the present study was to assess rTE and rTD of the dorsiflexors in young, healthy participants at $20 \%$, $60 \%$, and $100 \%$ of their maximal voluntary dorsiflexion torque (MVC) and activation prior to, 1 hour following, and 24 hours following 150 maximal eccentric dorsiflexion contractions. Nine participants $(6 \mathrm{M}, 3 \mathrm{~F} ; 23 \pm 2 \mathrm{yr})$ were seated in a dynamometer with their right hip and knee angle set to $110^{\circ}$ and $140^{\circ}$, respectively, with an ankle excursion set between $0^{\circ}$ and $40^{\circ}$ plantar flexion (PF). Custom electrical stimulation pads were placed on the tibialis anterior to elicit twitch doublets, as well as $10 \mathrm{~Hz}$ and $50 \mathrm{~Hz}$ tetanic contractions. Maximum voluntary contractions, twitch measures, and prolonged low frequency force depression were used to assess neuromuscular impairments. History dependent contractions consisted of a $1 \mathrm{~s}$ isometric $\left(40^{\circ} \mathrm{PF}\right.$ or $\left.0^{\circ} \mathrm{PF}\right)$ phase, a $1 \mathrm{~s}$ shortening or lengthening phase $\left(40^{\circ} / \mathrm{s}\right)$, and an $8 \mathrm{~s}$ isometric $\left(0^{\circ} \mathrm{PF}\right.$ or $\left.40^{\circ} \mathrm{PF}\right)$ phase. At 1- and 24-hours following exercise, respectively, MVC torque decreased $\sim 25 \%$ and $\sim 21 \%$, doublet twitch torque decreased $\sim 53 \%$ and $\sim 34 \%$, and low frequency force depression increased $\sim 54 \%$ and $\sim 22 \%$, indicating significant muscular impairment following the eccentric contractions. Prior to, 1 hour following, and 24 hours following exercise, rTD averaged across conditions was $\sim 9 \%, \sim 21 \%$, and $\sim 23 \%$, respectively, while rTE averaged across conditions was $\sim 16 \%, \sim 30 \%$, and $\sim 26 \%$, respectively. Thus, across rTD and rTE conditions there was a 2-fold increase following exerciseinduced weakness. It appears that a weakened neuromuscular system amplifies the magnitude of the history-dependence of torque. (Supported by NSERC)

\section{Vestibular control of standing balance following 24-hour sleep deprivation}

Paige V. Copeland, Megan L. Trotman, Hogun Kang, Chris J. McNeil, and Brian H. Dalton

School of Health and Exercise Sciences, UBC Okanagan, Kelowna, BC Canada.

Though the importance of sleep is well documented, sleep deprivation (SD) is common in today's society. Acute SD (e.g., $>24$ hours without sleep) is known to impact cognitive and sensorimotor function, including that of the vestibular system, as the vestibulo-ocular reflex is enhanced following SD. Further, SD can negatively affect postural control, though results are not conclusive, and the underpinning physiological mechanisms remain unclear. The purpose of this study was to examine the effect of 24-hour SD on the vestibular control of standing balance. It was hypothesized that after 24-hour $\mathrm{SD}$, vestibular-evoked balance responses would increase compared to a well-rested state. Under two conditions (i.e., following a night of typical sleep and 24-hour SD), nine healthy females (22.8 \pm 2.9 years) completed two 90-s trials of binaural, bipolar stochastic electrical vestibular stimulation $(\mathrm{EVS} ; 0-25 \mathrm{~Hz}$, root-mean-square $=1.1 \mathrm{~mA}$ ) and two 120-s trials of quiet stance. Mediolateral (ML) ground reaction forces were evaluated using a triaxial force plate and surface electromyography of the right medial gastrocnemius (MG) was sampled. The EVS signal was cross-correlated with the motor output signals (ML forces and MG EMG) in the frequency (gain and coherence) and time (cumulant density) domains to quantify vestibular control of balance. Overall, 24-hour SD did not alter quiet stance parameters $(p \geq 0.06)$. In the time domain, SD did not alter the EVS-MG $(p=0.51)$ nor the EVS-ML force $(p=0.06)$ cumulant density peak-to-peak amplitudes. Compared to the control, SD had limited effect on the EVS-MG and EVS-ML force coherence, but led to greater normalized gain amplitudes for EVS-MG $(<8,11-24 \mathrm{~Hz})$ and EVS-ML force $(0.5-9 \mathrm{~Hz})$. The current findings indicate that SD leads to higher sensitivity of the central nervous system when transforming vestibular-driven signals for standing balance control. (Supported by NSERC). 


\section{Ability of pre-season fitness to predict in-season on-ice} performance for male adolescent ice-hockey athletes

\author{
D.M. Cordingley, ${ }^{1,2}$ B.J. Avery, ${ }^{1,3}$ D. Ogborn, ${ }^{1,4,5}$ and S.M.B. McRae $e^{1,4,5}$ \\ ${ }^{I}$ Pan Am Clinic Foundation, Winnipeg, MB R3M 3E4, Canada. \\ ${ }^{2}$ Applied Health Sciences, University of Manitoba, Winnipeg, MB, Canada. \\ ${ }^{3}$ Faculty of Kinesiology and Recreation Management, University of Manitoba, Winnipeg, MB, Canada \\ ${ }^{4}$ Department of Surgery, University of Manitoba, Winnipeg, MB, Canada. \\ ${ }^{5}$ Department of Physical Therapy, College of Medical Rehabilitation, University of Manitoba, Winnipeg, MB, \\ Canada.
}

From youth leagues to the professional level, fitness testing is commonly performed to monitor physical development and talent identification purposes; however, it is unclear if results provide insight into on-ice performance for ice-hockey. The purpose of this study was to determine which, if any, fitness testing variables could predict on-ice performance of male adolescent ice-hockey athletes. One-hundred and thirty-eight (forwards, $n=92$; defense, $n=46$ ) toplevel competitive ice-hockey players between 12-17 years of age participated. Off-ice fitness testing was performed September 2019 to evaluate physical traits as well as athlete anaerobic and aerobic energy systems, strength, flexibility, agility and speed. On-ice data for goals, assists, games played, average points per game and penalty minutes were extracted from the league website. Stepwise multiple linear regressions were performed to generate models to predict each on-ice variable based on the off-ice testing results. For forwards, the $5-10-5$ shuttle explained $27 \%$ of the variance in assists $\left(\mathrm{R}_{\text {adj }}^{2}=0.27, \mathrm{~F}(1,68)=26.72, \mathrm{p}<0.001\right)$. Absolute average power output (PO) and the 5-10-5 shuttle predicted $54 \%$ of the variance in games played $\left(\mathrm{R}_{\text {adj }}^{2}=0.54, \mathrm{~F}(2,67)=38.55, \mathrm{p}<0.001\right)$, and absolute average PO and grip strength predicted $22 \%$ of the variance in penalty minutes $\left(\mathrm{R}^{2}{ }_{\text {adj }}=0.22, \mathrm{~F}(2,67)=10.58, \mathrm{p}<0.001\right)$. For defense, body mass predicted $22 \%$ of the variance in goals $\left(\mathrm{R}_{\text {adj }}^{2}=0.22, \mathrm{~F}(1,31)=9.83\right.$, $\mathrm{p}=0.004$ ), while body mass and push-ups predicted $27 \%$ of the variance in assists $\left(\mathrm{R}_{\text {adj }}^{2}=0.27, \mathrm{~F}(2,30)=6.92, \mathrm{p}=0.003\right)$. Absolute average PO explained $33 \%$ of the variance in games played $\left(\mathrm{R}^{2}{ }_{\mathrm{adj}}=0.33\right.$, $\mathrm{F}(1,31)=16.76, \mathrm{p}<0.001)$, while flexibility and push-ups accounted for $24 \%$ of the variance in average points per game $\left(\mathrm{R}^{2}{ }_{\mathrm{adj}}=0.24\right.$, $\mathrm{F}(2,30)=6.17, \mathrm{p}=0.006)$. All other independent variables were not predicted by off-ice data or the models accounted for $<10 \%$ of the variance. Off-ice fitness provides some insight into potential on-ice performance, but should not stand alone for decision making purposes.

\section{Can high cardiorespiratory and musculoskeletal performance coincide? An examination in female collegiate soccer players}

\section{C.J. Correa, ${ }^{1}$ A.D. Khan, ${ }^{2}$ B.D. Day, ${ }^{1}$ and A.S. Perrotta ${ }^{1}$}

${ }^{1}$ Department of Kinesiology, Langara College, Vancouver, BC, Canada.

${ }^{2}$ School of Kinesiology, Faculty of Education, The University of British Columbia, Vancouver, BC, Canada.

The physiological demands imposed on female soccer players to execute technical and tactical oriented activities rely heavily on cardiorespiratory and musculoskeletal function. Physiological indices, which have shown to be imperative, are aerobic power ( $\left(\mathrm{OO} 2_{\mathrm{MAX}}\right.$ ), vertical jump $(\mathrm{cm})$, speed $(\mathrm{m} / \mathrm{sec})$, acceleration $\left(\mathrm{m} / \mathrm{sec}^{2}\right)$ and muscular force $(\mathrm{N})$. This investigation examined the association between maximal cardiorespiratory and musculoskeletal performance in 17 female soccer players training as part of a collegiate soccer team. Participants were $19.4 \pm 0.94 \mathrm{yr}$ old, and weighed $61.1 \pm 7.1 \mathrm{~kg}$, with a height of $168.4 \pm 5.0 \mathrm{~cm}$, a body composition of $25.7 \pm 5.5 \%$ body fat and a $\dot{\mathrm{V} O} 2_{\mathrm{MAX}}$ of $44.4 \pm 2.1 \mathrm{~mL} \cdot \mathrm{kg}^{-1} \cdot \mathrm{min}^{-1}$. Maximal cardiorespiratory performance was examined using the YoYo Intermittent Recovery Test Level 1. The final distance over the last completed shuttle was used to calculate $\dot{\mathrm{VO}}_{2 \mathrm{MAx}}$. Each participant performed three separate squat jumps (SJ) and counter movement jumps (CMJ) with their hands placed on their hips while on top of a force plate (Hawkin Dynamics Force Plate, ME, USA). The largest force $(\mathrm{N})$ production from each jump was included for analysis. Sprint performance was examined using speed $(\mathrm{m} / \mathrm{sec})$ and acceleration $\left(\mathrm{m} / \mathrm{sec}^{2}\right)$ over $5 \mathrm{~m}$ and $10 \mathrm{~m}$ distances, starting from a stationary position, with the use of timing gates (Brower Timing Systems, Draper, USA). The fastest sprint from three attempts were included for analysis. Normality of data sets were examined using a Shapiro-Wilk's test for normality $(\mathrm{p}>0.05)$. A Pearson correlation coefficient $(\mathrm{r})$ was utilized to examine the association between indices of musculoskeletal and cardiorespiratory performance. Significance was set at $(\mathrm{p}<0.05)$. This study was approved by and followed the recommendations of the Langara College Research Ethics Board. Significant $(\mathrm{p}<0.05)$ inverse associations were observed between YoYo performance and; $5 \mathrm{~m}$ speed $(\mathrm{r}=-0.60), 10 \mathrm{~m}$ speed $(\mathrm{r}=-0.51), 5 \mathrm{~m}$ acceleration $(\mathrm{r}=-0.60)$ and $10 \mathrm{~m}$ acceleration $(\mathrm{r}=-0.51)$. No significant associations $(\mathrm{p}>0.05)$ were observed between YoYo performance and; vertical jump $(\mathrm{r}=0.08), \mathrm{CMJ}(\mathrm{r}=-0.36)$ and $\mathrm{SJ}$ $(\mathrm{r}=0.02)$. Maximal musculoskeletal and cardiorespiratory performance may be polarized in female collegiate soccer players.

\section{The relationship between single muscle fibre and voluntary rate} of force development

B. Dalton, N. Mazara, M.I.B. Debenham, D.P. Zwambag, A.M. Noonan, E. Weersink, S.H.M. Brown, and G.A. Power

Department of Human Health and Nutritional Sciences, College of Biological Sciences, University of Guelph, Guelph, Ontario, Canada.

It has been suggested that the early phase of force development during a muscle contraction $(\leq 50 \mathrm{~ms})$ is associated with intrinsic contractile properties, while the late phase $(\geq 100 \mathrm{~ms})$ is associated with maximal force. There has been no direct investigation of single muscle fibre contractile properties and their relationship with whole muscle rate of force development (RFD). Therefore, we investigated the relationship between voluntary RFD and single muscle fibre RFD. Sixteen healthy, young (YM; $\mathrm{n}=8 ; 26.4 \pm 1.5 \mathrm{yrs}$ ) and older $(\mathrm{OM} ; \mathrm{n}=8 ; 70.1 \pm 2.8 \mathrm{yrs})$ males performed maximal voluntary isometric contractions and electrically evoked twitches of the knee extensors on a HUMAC NORM dynamometer. Voluntary RTD was calculated over 5 time epochs: $0-30,0-50,0-100,0-200$, and $0-$ $500 \mathrm{~ms}$. Percutaneous muscle biopsies were taken from the vastus lateralis and chemically permeabilized. Single muscle fibres were mounted between a force transducer and length controller, and maximally activated. Single fibre RFD was assessed using a slacken re-stretch method, which dissociates all cross-bridge's, and the rate at which force is re-developed is the transition from non-force bearing to force bearing cross-bridge states, allowing for the assessment of cross-bridge state transitional kinetics. Following mechanical testing, muscle fibres were fibre typed using SDS-PAGE. As expected, there were stronger relationships between Twitch RTD and early Voluntary RTD as compared to later time epochs. As well, it appears that the relationship between single fibre RFD and Voluntary RTD was strongest at the early time epochs $(\leq 50 \mathrm{~ms})$ and decreased with increasing time epoch. Analysis is ongoing to investigate the effects of age and fibre type, but based on these data, it appears that early RTD during voluntary contractions is, in part, related to basic single muscle fibre intrinsic contractile properties. (Supported by NSERC)

Vertical force output does not govern sprint performance in female college soccer players

\section{B.D. Day, ${ }^{1}$ C.J. Correa, ${ }^{1}$ A.D. Khan, ${ }^{2}$ and A.S. Perrotta ${ }^{1}$}

${ }^{I}$ Department of Kinesiology, Langara College, Vancouver, BC, Canada

${ }^{2}$ School of Kinesiology, Faculty of Education, The University of British Columbia, Vancouver, BC, Canada.

Soccer is a sport that requires its athletes to have high levels of musculoskeletal power to perform various skills required throughout a match. Key performance indicators routinely examined are 
explosive vertical and horizontal movements, typically performed using jumping and sprinting assessments. This investigation examined the association between indices of vertical force and sprint performance in 17 female soccer players training as part of collegiate soccer team. Participants were $19.4 \pm 0.94 \mathrm{yr}$ old, and weighed $61.1 \pm 7.1 \mathrm{~kg}$, with a height of $168.4 \pm 5.0 \mathrm{~cm}$ and a body composition of $25.7 \pm 5.5 \%$ body fat. Each participant performed three separate squat jumps (SJ) and counter movement jumps (CMJ) with their hands placed on their hips while on top of a force plate (Hawkin Dynamics Force Plate, ME, USA) The largest force $(\mathrm{N})$ production from each jump was included for analysis. Sprint performance was examined using speed $(\mathrm{m} / \mathrm{sec})$ and acceleration $\left(\mathrm{m} / \mathrm{sec}^{2}\right)$ over $5 \mathrm{~m}$ and $10 \mathrm{~m}$ distances, starting from a stationary position, with the use of timing gates (Brower Timing Systems, Draper, USA). The fastest sprint from three attempts were included for analysis. Normality of data sets were examined using a Shapiro-Wilk's test for normality $(\mathrm{p}>0.05)$. A Pearson correlation coefficient $(\mathrm{r})$ was utilized to examine the association between indices of vertical force and sprint performance. Significance was declared using a probability of $(\mathrm{p}<0.05)$. This study was approved by and followed the recommendations of the Langara College Research Ethics Board. All associations between indices of vertical force and sprint performance were insignificant and ranged between $(\mathrm{r}=-0.10, \mathrm{p}>0.90)$ and $(\mathrm{r}=0.09, \mathrm{p}>0.90)$. Coaches are encouraged not to equate vertical and horizontal physical performance measures in female college soccer players. This evidence supports practitioners to administer exercises utilizing horizontal explosive movements for enhancing sprint performance.

Frequency and time domain responses of vestibular-evoked balance reflexes following foot dorsum cooling

Mathew I.B. Debenham, ${ }^{1}$ Hogun J. Kang, ${ }^{1}$ Stephen S. Cheung, ${ }^{2}$ and Brian H. Dalton ${ }^{1}$

${ }^{I}$ School of Health and Exercise Science, The University of British Columbia, Kelowna, BC, Canada. ${ }^{2}$ Department of Kinesiology, Brock University, St. Catharines, ON, Canada.

Somatosensory feedback from the feet can provide important information for maintaining standing balance. The vestibular control of balance is enhanced following foot sole cooling owing to alterations in cutaneous mechanoreceptor function. The purpose here was to determine whether decreasing cutaneous receptor sensitivity via foot dorsum cooling modulates the vestibular control of balance. We hypothesized that vestibular-evoked balance responses would increase with foot cooling. Eighteen participants $(9$ males; 9 females) stood quietly on a force plate with feet together, eyes closed, and head rotated leftward during 4 trials ( 2 baseline; 2 cooled) of binaural, bipolar electrical vestibular stimulation (EVS; $0-25 \mathrm{~Hz}$; peak-to-peak amplitude $= \pm 5 \mathrm{~mA}$; $90 \mathrm{~s}$ each). The cooling protocol involved placing icepacks on the dorsum of both feet for at least 15min. During the EVS trials involving cooling, the icepacks remained on the feet. Monofilament testing was performed at three locations (Upper, Middle and Lower) on the foot dorsum before and after cooling to determine tactile detection threshold. Vestibularevoked balance responses were characterized using a correlationlike analysis to determine the relationship between the EVS input and the whole-body balance responses (anterior posterior forces; APF) in the time (cumulant density) and frequency (coherence and gain) domains. T-type thermocouples monitored foot dorsum skin temperature, which decreased $\left(28.0 \pm 1.9^{\circ} \mathrm{C}\right.$ to $\left.8.1 \pm 3.4^{\circ} \mathrm{C} ; \mathrm{p}<0.05\right)$ concomitantly with an increase in tactile detection thresholds - indicating impaired perception - following cooling $(\mathrm{p}<0.05)$. In contrast to our hypothesis, cooling reduced EVS-APF peak-to-peak amplitude compared to baseline $(0.19 \pm 0.04$ vs $0.21 \pm 0.05$, respectively; $\mathrm{p}<0.05)$ in the time domain. The EVS-APF coherence and gain were significantly reduced following cooling compared to control trials at multiple frequencies (Coherence: females: $<13 \mathrm{~Hz}$, males: $<9 \mathrm{~Hz}$; Gain: males and females: $<4 \mathrm{~Hz}$ ). These results likely represent an alteration in cutaneous mechanoreceptor sensitivity of the foot dorsum and emphasize a location-dependent modification of foot skin afferent feedback on vestibular control of standing balance. (Supported by NSERC)

\section{Implementation of Teleprehabilitation for Onco-Surgical Candidates during Covid-19 Pandemic: A Retrospective Pilot Cohort}

K. Drummond, ${ }^{1,2}$ G. Lambert, ${ }^{1,2}$ B. Tahasildar, ${ }^{1}$ and F. Carli ${ }^{1,2}$

${ }^{I}$ Department of Anaesthesia, McGill University, Montreal, Quebec, Canada

${ }^{2}$ Department of Experimental Surgery, McGill University, Montreal, Quebec, Canada.

The Covid-19 pandemic has changed the way public health systems operate, which had deleterious effect on cancer care, and more specifically preoperative support. This study aimed to document 1) the teleprehabilitation program received by oncologic surgical candidates, 2) the patients' perspectives of the technology-assisted program, and 3) their preoperative functional trajectories. This retrospective pilot-cohort study included ten adults scheduled for elective cancer surgery, who were referred by their surgeons to receive teleprehabilitation. Data was acquired through patient charts in addition to virtual physical activity monitoring platforms. Ten patients (8 males; mean age: 68.3, SD: 11.96) diagnosed with various thoraco-abdominal malignancies were included in the current descriptive study. The preoperative period differed greatly between patients (median: $9.5[2-15]$ weeks), most patient received neoadjuvant therapies $(n=7)$. All patients received teleprehabilitation services following diagnosis until their respective surgeries. The successes identified were related to the recruitment and assessment, the clinic scheduling and interventions, and the optimal medical follow-up. The challenges identified were related to the program costs, the patients' learning curve, acquisition of the physical activity data, the adjustment of the multidisciplinary team to new technologies, and the postoperative loss to follow-up. Patient were satisfied with the teleprehabilitation program (i.e., services delivered) reporting an average appreciation score of $96 \%$, and they perceived the technologies provided to be $90 \%$ user-friendly. Further, patients' functional capacities did not change significantly during the preoperative period, with only the timed-up and go and the sit-to-stand tests showing trends toward improvement. The findings of the current study are paramount in view of the current international health paradigm changes prioritizing remote interventions facilitated through digital communication technologies. It provides important insight into the clinical application of telehealth, with elderly populations, notably in the context of acute preoperative cancer care. The growing body literature has emphasized a lack of evidence pertaining to virtually assisted rehabilitation programs and their potential for implementation in both older adults and acute care. The current research provides a preliminary framework for other institutions that may wish to implement teleprehabilitation programs.

\section{Prehabilitation to Enhance Esophageal Cancer Care: Preoperative Functional Outcomes}

\author{
K. Drummond, ${ }^{1}$ E.M. Minnella, ${ }^{2,3}$ L. Ferri, ${ }^{4}$ and F. Carli ${ }^{3}$ \\ ${ }^{I}$ Experimental Surgery, McGill University, QC H3A OG4 \\ ${ }^{2}$ Department of Anesthesia, McGill University, QC H3A OG4 \\ ${ }^{3}$ Department of Anaesthesia and Intensive Care, IRCCS San Raffaele Scientific Institute, Milano 60, 20132, Italy. \\ ${ }^{4}$ Department of Surgery \& Oncology, McGill University, QC H3A OG4.
}

Esophageal cancer (EC) is a morbid disease associated with a poor clinical prognosis. Surgery preceded by neoadjuvant chemotherapy (NACT) have become central to disease management. Unfortunately, the cancer therapies often lead to declines cardiorespiratory fitness and functional status throughout the preoperative period, in-turn increasing surgical risks. Importantly, prehabilitation involves the delivery of multimodal (exercise, nutrition, stress reduction) support 
with the aim improving patient trajectories. Despite the compelling rationale, few studies have investigated its potential in these high-risk patients, nevertheless during NACT. The current research describes a pilot study aimed to investigate the effects of a multimodal prehabilitation program in patients with EC. Notably, to investigate its safety, feasibility and to document its impacts on functional status. In the study 24 patients with locally advanced EC were enrolled to the current study, of whom, all but-one completed the program, performing both evaluations (baseline and preop) and received their respective surgeries. The program's feasibility was dependent on the cohort's overall self-reported adherence to each component of the program including exercise and nutrition. Patients performed a comprehensive test battery at baseline and preoperatively to assess their changes in anthropometrics, functional capacity, and strength. Only one patient dropped out of the study. The cohort's median overall program adherence rate was $72.7 \%$ and that of the exercise components was comparable to the literature ( $68.7 \%$ vs $70 \%, \mathrm{p}=0.456)$. Regarding preoperative changes in functional fitness, cardiorespiratory function did not change, as reflected by the 6-MWT $\triangle 6-\mathrm{MWD},+1 \mathrm{~m}$; $\mathrm{p}=0.796)$ and CPET $\left(\Delta \dot{\mathrm{VO}}_{2} \mathrm{AT},+0.4 \mathrm{ml} / \mathrm{kg} / \mathrm{min} ; \mathrm{p}=0.39\right)$ conversely the number of timed-repetitions performed during the sit-to-stand test increased by $25 \%(\mathrm{p}=0.002)$. Patients also noted significant improvements in their self-reported physical activity levels, quality of life and disease-related symptom burden. The findings demonstrated that personalized multimodal prehabilitation programs could be feasibly delivered to patients with EC during NACT. While the cohort experienced modest functional changes, it suggests that prehabilitation may have played a role in preserving functional fitness throughout the preoperative period and preventing the decline otherwise expected. (Supported by the Rossy Cancer Network)

\section{A multi-method approach to adapt adverse event reporting guidelines to resistance training studies}

R. El-Kotob, ${ }^{1,2}$ J.R. Pagcanlungan, ${ }^{1}$ B.C. Craven, ${ }^{2,3,4,5}$ C. Sherrington, ${ }^{6,7}$ M. Mourtzakis, ${ }^{1}$ and L.M. Giangregorio ${ }^{1,8}$

${ }^{I}$ Department of Kinesiology and Health Sciences, Faculty of Health, University of Waterloo, Waterloo, ON, N2L 3GI, Canada.

${ }^{2}$ KITE at Toronto Rehabilitation Institute, University Health Network, Toronto, ON, M4G 3V9, Canada. ${ }^{3}$ Rehabilitation Sciences Institute, School of Graduate Studies, University of Toronto, Toronto, ON, M5G 1V7, Canada.

${ }^{4}$ Department of Medicine, Division of Physical Medicine and Rehabilitation, University of Toronto, Toronto, ON, M5S 3H2, Canada.

${ }_{5}^{5}$ Institute of Health Policy, Management and Evaluation, School of Graduate Studies, University of Toronto, Toronto, ON, M5T 3M6, Canada.

${ }^{6}$ Institute for Musculoskeletal Health, Faculty of Medicine and Health, University of Sydney, NSW 2006, Sydney, Australia.

${ }^{7}$ School of Public Health, Faculty of Medicine and Health, University of Sydney, Sydney, NSW 2006, Australia. ${ }^{8}$ Schlegel-University of Waterloo Research Institute for Aging, University of Waterloo, Waterloo, ON, N2J OE2, Canada.

Adverse events (AEs) are not being consistently monitored or reported in resistance training (RT) studies. We took a multimethod approach to: 1) explore the experiences and perspectives of individuals with chronic health conditions who had an $\mathrm{AE}$ as a result of RT; 2) understand researchers' current practices and perspectives on $\mathrm{AE}$ reporting in $\mathrm{RT}$, and identify barriers and facilitators of $\mathrm{AE}$ reporting; and 3) generate exercise-specific AE-reporting recommendations. Web conference or telephone-based one-on-one semistructured interviews were conducted with people who have chronic health conditions and had an AE as a result of RT ( $n=12)$, and researchers who published RT studies $(\mathrm{n}=14)$. Interview data were analyzed using the thematic framework method. AE-reporting recommendations were generated based on interview data and were turned into an electronic survey to perform a modified Delphi process involving 19 international researchers who published RT studies. We learned that despite participant awareness of the value and benefits of RT, there is concern about experiencing exerciserelated AEs. Furthermore, the perceived risks of RT influenced the participants' decision to engage or return to RT. Within the exer- cise community, there is suboptimal implementation of existing $\mathrm{AE}$ reporting standards, or the perception that the available guidelines do not apply to exercise trials. The barriers identified were that researchers lack guidance, resources, or motivation for rigorous $\mathrm{AE}$ reporting. To facilitate $\mathrm{AE}$ reporting, researchers educate and value participants, use trained personnel, and implement standardized guidelines. An exercise-specific AE-reporting toolkit (i.e., checklist, template form, and decision tree) was developed based on the Delphi results (3 rounds; minimum 74\% agreement on each recommendation). Overall, accurate AE reporting will allow people with common health conditions, researchers, and health care providers to make evidence-based decisions as to whether the benefits of RT truly outweigh the harms.

\section{Carbohydrate-energy replacement following low-volume high-intensity interval exercise blunts next-day glycemic control in women}

\section{S. Estafanos, B. Friesen, A. Govette, and J.B. Gillen}

Department of Kinesiology, University of Toronto, Toronto, ON M5S2C9, Canada.

Improvements in insulin sensitivity and glycemic control have been observed for $\sim 24 \mathrm{hr}$ following a single session of low-volume high-intensity interval exercise (HIIE), but it is unclear if this is a direct effect of exercise or an indirect effect of the exercise-induced energy deficit. The purpose of this study was to investigate the effect of carbohydrate-energy replacement after a single session of HIIE on $24 \mathrm{hr}$ glycemic control in women. In a randomized order, seven recreationally active women completed 3 trials in the midfollicular phase of the menstrual cycle: 1) No exercise (CTL); 2) HIIE + post-exercise carbohydrate replenishment (EX-HC); 3) HIIE + postexercise carbohydrate restriction (EX-LC). On day 1, participants were fitted with a continuous glucose monitor (CGM) and performed a single session of HIIE (10x1 min @ 90\% HRmax; EX-HC and EX-LC) or remained seated (CTL) in the lab at $1700 \mathrm{~h}$. Post-exercise, EX-HC consumed a carbohydrate beverage equivalent to their exerciseinduced energy expenditure ( $43 \pm 10 \mathrm{~g}$ carbohydrate); EX-LC ingested a taste-matched non-caloric placebo. All groups consumed a lowcarbohydrate dinner (5/79/16\% carbohydrate/fat/protein) $2 \mathrm{hr}$ later. The following morning, participants consumed $75 \mathrm{~g}$ of glucose as 'breakfast', followed by consumption of 3 mixed-macronutrient meals (snack, lunch, dinner; 55/30/15\% carbohydrate/fat/protein) under freeliving conditions. CGM-derived glucose means and area under the curve (AUC) were determined for $3 \mathrm{~h}$ postprandial periods and over $24 \mathrm{hr}$ post-exercise. Postprandial glycemia following breakfast and snack were reduced in EX-LC compared to EX-HC, as reflected by lower $3 \mathrm{hr}$ glucose mean (breakfast: $5.5 \pm 0.5$ vs. $6.7 \pm 1, p=0.01$, Cohen's $d=1.4$; snack: $4.9 \pm 0.3$ vs. $5.7 \pm 0.8, p=0.02, d=1.4$ ) and/or AUC (breakfast: $994 \pm 86$ vs. $1208 \pm 190, p=0.01, d=1.5)$. Postprandial glycemia following lunch and dinner were not different between conditions $(p>0.05)$. There was a large effect size $(d=1.1)$ for a reduction in $24 \mathrm{hr}$ mean glucose in EX-LC (5.2 \pm 0.3$)$ vs. EX-HC (5.7 \pm 0.7$)$, which approached significance (ANOVA $p=0.054$ ). Our findings suggest that the glycemic benefits of low-volume HIIE in women are dependent on maintaining the carbohydrate-energy deficit post-exercise.

\section{Resistance exercise training induces load specific absolute muscle endurance gains in young women: a within-participant randomized controlled trial}

M.D. Fliss, J. Stevenson, S. Mardan-Dezfouli, D.C.W. Li, and C.J. Mitchell

School of Kinesiology, The University of British Columbia, Vancouver, BC, Canada.

Local muscle endurance is typically defined as relative endurance, the number of repetitions which can be performed with a given percentage of an individual's current one repetition maximum 
(1RM). Lower load (LL) resistance exercise training (RET) induces superior gains in relative endurance compared higher load (HL) RET, largely due to blunted 1RM gains. Absolute endurance is defined as the number of repetitions which can be completed with a given absolute load. The purpose of this trial was to examine how HL and LL RET impact changes in absolute endurance when it is assessed using both a heavier and lighter external resistance. A repeated measures within-participant unilateral design was employed. Each participant trained knee extensions (KE) thrice weekly for 10 weeks performing three sets to the point of volitional fatigue within 6-12 repetitions (HL leg) and 20-30 repetitions (LL leg). Absolute endurance was assessed as the number of repetitions which could be completed with 30 and $80 \%$ of pre-training 1RM. HL RET induced a greater increase in KE 1RM compared to LL RET (HL = $12.1 \pm 4.9 \mathrm{~kg}, \mathrm{LL}=7.8 \pm 5.5 \mathrm{~kg}, P<0.01)$. LL RET showed a larger increase in lighter weight absolute endurance compared to HL RET ( $\mathrm{HL}=17.4 \pm 17$ repetitions, $\mathrm{LL}=25.1 \pm 20.2$ repetitions, $P=0.02$ ), while HL RET showed a larger increase in heavier weight absolute endurance $(\mathrm{HL}=9.6 \pm 4.5$ repetitions, $\mathrm{LL}=7.1 \pm 5$ repetitions, $P=0.02$ ). Based on these findings, it would be advised that clinicians/trainers prescribe a combination of HL and LL RET to improve absolute endurance across a spectrum of loads. These results highlight two distinct mechanisms of adaptation, increasing $1 \mathrm{RM}$ results in pre-training loads representing a lower percentage of $1 \mathrm{RM}$ post training thus requiring lower motor unit activation and providing a greater neuromuscular reserve at a given absolute load. This likely contributes to adaptions in both LL and HL legs and explains the superiors heavy load absolute endurance in the HL leg. Lighter weight absolute endurances improved more in the LL leg despite lower 1RM gains suggesting increased glycolytic enzyme content, improved buffering capacity, or increased mental resilience were responsible.

\section{The acute effects of post-exercise milk consumption on systemic inflammation after combined resistance and plyometric exercise in young females}

Emily C. Fraschetti, Lauren E. Skelly, Ali A. Abdul-Sater, and Andrea R. Josse

School of Kinesiology and Health Science, Faculty of Health, York University, Toronto, ON M3J IP3, Canada.

The post-exercise provision of milk and dairy protein has been used to stimulate muscle protein synthesis, reduce muscle soreness, and improve subsequent athletic performance. Post-exercise inflammation may modulate these processes; however, little research has been done to determine the influence of whole-food, white milk consumption on the post-exercise inflammatory response. Using a crossover design, the aim of the present study was to characterize and compare the inflammatory response following the consumption of white milk ( $0 \% \mathrm{MF})$ vs. an isoenergetic, isovolumetric carbohydrate control beverage ( $\mathrm{CHO}$; maltodextrin and water) after a single session of combined resistance and plyometric exercise in young, recreationally active, healthy females $(n=13$; age: $20 \pm$ 2.3 years; BMI: $21.0 \pm 1.1 \mathrm{~kg} / \mathrm{m}^{2}$ ). Participants reported to the lab after an overnight fast and underwent the acute exercise bout, followed by consumption of the first trial drink, either $555 \mathrm{~mL}$ of skim white milk or the carbohydrate control. A second, identical trial drink was consumed $1 \mathrm{~h}$ post-first trial drink. Blood samples were taken at baseline, $15 \mathrm{~min}, 75 \mathrm{~min}, 24 \mathrm{~h}$ and $48 \mathrm{~h}$ post-exercise, and serum concentrations of interleukin-6 (IL-6), tumor necrosis factoralpha (TNF- $\alpha$ ), interleukin-1 $\beta$ (IL-1 $\beta$ ), and interleukin-10 (IL-10) were assessed. Two-way repeated-measures ANOVAs (within-factor time: baseline, $15 \mathrm{~min}, 75 \mathrm{~min}, 24 \mathrm{~h}, 48 \mathrm{~h}$ post-exercise; withinfactor trial: MILK, CHO) were conducted on the absolute concentrations. IL-6 increased significantly at $15 \mathrm{~min}$ post-exercise (vs. baseline) and returned to baseline by $75 \mathrm{~min}$ in both groups (main time effect, $\mathrm{p}=0.02$ ). Between 24 and $48 \mathrm{~h}$ post-exercise, IL-10 concentrations changed in opposite directions (interaction, $\mathrm{p}=0.02$ ), with concentrations decreasing (from being higher at $24 \mathrm{~h}$ ) in MILK and increasing (from being lower at $24 \mathrm{~h}$ ) in CHO. There were no significant effects for TNF- $\alpha$ or IL-1 $\beta$. Post-exercise white milk consumption did not acutely influence pro-inflammatory cytokines following resistance and plyometric exercise in young females; however, there were divergent responses between 24 and $48 \mathrm{~h}$ for the anti-inflammatory cytokine, IL-10, which warrants further exploration.

Feasibility and utility of wearable technology in remote exercise programs

J. Fraser, N. Carraretto, C.A. Russell, H. Gibson, L.A. Kraemer, S. Pitton, E. Smith, J. Stevens, and M. Mourtzakis

Centre for Community, Clinical and Applied Research Excellence, Department of Kinesiology and Health Sciences, University of Waterloo, Waterloo, ON N2L 3G6, Canada.

The Centre for Community, Clinical and Applied Research Excellence (CCCARE) offers individualized, structured exercise programs including UW WELL-FIT, STEPS, and Brain and Body, developed for individuals with cancer, stroke, and dementia, respectively. Historically, these programs have relied on subjective, self-reports to gather information related to the amount and type of physical activity performed in daily life, which has known limitations. In addition, the COVID-19 pandemic and corresponding shift to remotebased delivery models has limited the ability to collect objective physiological measurements such as heart rate. Wearable devices offer the ability to objectively measure the amount and intensity of physical activity both during structured exercise and in daily life in addition to collecting accurate heart rates. A pilot trial exploring the feasibility and utility of integrating the ActiGraph GT9X and Polar $\mathrm{H} 7$ heart rate monitors into remote exercise programming is in progress. Bluetooth ${ }^{\circledR}$ pairing the wrist-worn ActiGraph watch to the Polar chest strap offers the utility of real-time heart rate monitoring for clients during structured exercise and the ability for staff to accurately prescribe intensity levels when worn during remote assessments. Along with real-time applications, raw data can be extracted for custom analysis or using ActiGraph proprietary filtering methods and publicly available algorithms to provide a variety of outputs. Results from the pilot study include validation testing of ActiGraph step counts worn at different body locations (hip, ankle, and wrist) under different continuous gait and structured exercise conditions, evaluation of data extraction and filtering processes, and commentary on weartime protocol, clinical and participant report generation, and usability feedback from both program participants and staff. Through sharing our lessons learned of incorporating wearables into remote exercise programming, we hope to better understand how they can advance clinical care both for remote and in-person exercise delivery models.

Achilles Tendon Area Is Not a Predictor of Tibia Bone Strength:

\section{J. Gough, ${ }^{1}$ J.D. Johnston, ${ }^{2}$ and S.A. Kontulainen ${ }^{1}$}

${ }^{I}$ College of Kinesiology, University of Saskatchewan, Saskatoon, SK S7N 5B2, Canada. ${ }^{2}$ College of Engineering, University of Saskatchewan, Saskatoon, SK S7N 5A9, Canada.

Imaged muscle area is commonly used as a surrogate of muscle force when assessing bone adaptation to loading. The role of tendon area on bone strength, however, is poorly understood. Our objective was to test the hypothesis that Achilles tendon area independently predicts variance in tibia bone strength. We acquired pQCT scans from the lower legs of 901 volunteers (631 female, age range from 6-90 yrs). We characterized Achilles tendon area at the ankle, lower leg muscle area at the calf and estimated bone strength at the distal (BSIc) and shaft (SSIp) sites of tibia. We first built sex-specific linear regression base models (including age, height, and body weight) to predict variance in bone strength. We 
then included Achilles area with and without muscle area, to the base models. Base models including muscle area explained most of the variance (49-85\%) in bone strength at the distal and shaft sites of tibia. For both females and males, Achilles tendon area did not improve the fit of the models ( $\mathrm{R}^{2}$ did not change) or independently predicted variance in bone strength at either site, with or without muscle area ( $\beta$-coefficients ranged from 0.01 to $0.04, \mathrm{p}>0.05$ ). Results suggest that Achilles tendon area cannot replace or complement muscle area as a surrogate estimate of muscle force when estimating loading stimulus on bone. Since tendons transmit forces from muscle to bone, our findings suggest that tendon area may be the weak link in bone-muscle interplay. This finding warrants further investigation as it may contribute to the etiology underpinning tendon injuries.

\section{Effect of at-home bodyweight interval exercise before or after breakfast on postprandial glycemic control in females}

\section{A. Govette and J.B. Gillen}

Faculty of Kinesiology \& Physical Education, University of Toronto, Toronto, ON M5S 2W6, Canada.

Previous research has demonstrated that a single session of lowvolume high-intensity interval exercise (10x1 min cycling sprints at $\sim 90 \% \mathrm{HR}_{\max }$, with 1 min recovery), improves glycemic control in healthy adults and those at risk for metabolic disease. However, most studies have been performed under controlled laboratory settings and utilized specialized exercise equipment, which may limit ecological validity. Moreover, the timing of meals around exercise may influence the glycemic effects of low-volume interval exercise, but this has yet to be explored. The purpose of this study was to investigate the effects of a single session of home-based bodyweight interval exercise (BWI), performed before or after a meal, on postprandial glycemia. In a randomized order, 10 female participants (age: $23 \pm 2$ yr; BMI: $22 \pm 2 \mathrm{~kg} / \mathrm{m}^{2}$; $\dot{\mathrm{V}}_{2}$ peak (estimate): $46 \pm 1 \mathrm{~mL} / \mathrm{kg} / \mathrm{min}$ ) completed two virtually-monitored trials in the follicular phase of the menstrual cycle. Participants performed high-intensity BWI by following a pre-recorded video (10x1 min of whole-body exercises, $1 \mathrm{~min}$ recovery) either $5 \mathrm{~min}$ before (EX-FED) or $10 \mathrm{~min}$ after (FED-EX) a mixed-macronutrient breakfast $(470 \mathrm{kcal}, 69 \%$ carbohydrate, $13 \%$ fat, $18 \%$ protein). Heart rate and rating of perceived exertion (RPE) were measured during exercise, and repeated capillary blood samples were obtained for $2 \mathrm{hr}$ postprandial for determination of glucose mean, peak and area under the curve (AUC). The postprandial glycemic excursion was reduced in FED-EX relative to EX-FED as reflected by a lower glucose mean ( $6.1 \pm 0.6$ vs. $6.8 \pm 0.8, \mathrm{p}=0.02)$, peak ( $7.4 \pm 1.2$ vs. $8.5 \pm 1.5$, $\mathrm{p}=0.014)$ and AUC $(758 \pm 72$ vs. $973 \pm 82, \mathrm{p}<0.001)$. Exercise-induced heart rate (FED-EX: $86 \pm 6$ vs. EX-FED: $86 \pm 4 \% \mathrm{HR}_{\max }, \mathrm{p}=0.96$ ) and RPE (FED-EX: $14 \pm 2$ vs. EX-FED: $15 \pm 2$, p=0.06) were within the high-intensity domain and similar between conditions. BWI can be effectively performed athome without the need for specialized exercise equipment. Performing BWI after a mixed-macronutrient breakfast reduced meal-induced glycemia compared to when the same exercise was performed before the meal. Our findings have implications for optimizing exercise-nutrient timing recommendations for improved glycemic control in females.

\section{Effect of menstrual cycle on markers of bone metabolism at rest} and in response to exercise in eumenorrheic females

Anne Guzman, ${ }^{1}$ Nigel Kurgan, ${ }^{1}$ Seth F. McCarthy, ${ }^{2}$ Sara C. Moniz, ${ }^{2}$ Tom J. Hazell, ${ }^{2}$ and Panagiota Klentrou ${ }^{1}$

${ }^{1}$ Department of Kinesiology, Brock University.

${ }^{2}$ Department of Kinesiology, Wilfrid Laurier University.

This study examined potential fluctuations in bone metabolic markers across the menstrual cycle at rest and after 30 minutes of vigorous-running at $80 \%$ of $\mathrm{VO}_{2}$ max. Resting and post-exercise $(0$,
30, $90 \mathrm{~min}$ ) sclerostin (bone formation inhibitor), parathyroid hormone (PTH), carboxy-terminal cross-linking telopeptide of type I collagen ( $\beta$-CTX, bone resorption marker), and procollagen type $1 \mathrm{~N}$ propeptide (P1NP, bone formation marker) were assessed in 10 young, recreationally active eumenorrheic women $(21.7 \pm 3.2$ years, $23.2 \pm 3 \mathrm{~kg} / \mathrm{m}^{2}$ ) during the mid- to late-follicular (FP: day $8.0 \pm 1.4$ ) and mid-luteal (LP: day $22.0 \pm 2.5$ ) phases of the menstrual cycle. At rest, there were no differences in sclerostin (FP: $266.5 \pm 48.6 \mathrm{pg} / \mathrm{mL}$; LP: $296.0 \pm 37.5 \mathrm{pg} / \mathrm{mL} ; \mathrm{P}=0.507)$, PTH (FP: $1.00 \pm 0.22 \mathrm{pmol} / \mathrm{L} ; \mathrm{LP}$ : $0.71 \pm 0.16 \mathrm{pmol} / \mathrm{L} ; P=0.485), \beta$-CTX (FP: $243.1 \pm 52.7 \mathrm{ng} / \mathrm{mL}$; LP: $202.4 \pm 30.8 \mathrm{ng} / \mathrm{mL} ; P=0.691$ ), or P1NP (FP: $56.9 \pm 11.30 \mathrm{ng} / \mathrm{mL}$; LP: $64.30 \pm 18.32 \mathrm{ng} / \mathrm{mL} ; P=0.133$ ) between phases. As there were no main effects for menstrual phase and no interaction, post-exercise responses did not differ between menstrual phases for any of the markers $(P>0.05)$. Significant time effects were found in sclerostin, $\beta$-CTX and P1NP, but not in PTH. Sclerostin increased from pre- to 0 min post-exercise (+46\%; $P<0.0001)$, then returned to resting levels at $30 \mathrm{~min}$ post-exercise. P1NP increased immediately post-exercise $(+29 \% ; P<0.0001)$, returning to resting levels at $30 \mathrm{~min}$ post-exercise. $\beta$-CTX decreased from pre- to $0 \mathrm{~min}$ post-exercise $(-20 \% ; P=0.004)$ and remained below its pre-exercise levels at $30 \mathrm{~min}$ post-exercise $(-12 \%$; $P=0.039)$ and $90 \mathrm{~min}$ post-exercise $(-17 \% ; P=0.002)$. These results demonstrate that sclerostin, PTH, $\beta$-CTX and P1NP do not differ at rest or in response to exercise across the menstrual cycle.

Enhanced vestibular-evoked balance responses may compensate for age-related reductions in peak power

\author{
Phuong L. Ha, ${ }^{1}$ Wendy B. Peters, ${ }^{2}$ Michael A. McGeehan, ${ }^{2}$ \\ and Brian H. Dalton ${ }^{1,2}$ \\ ${ }^{I}$ School of Health and Exercise Sciences, The University of British Columbia Okanagan, Kelowna \\ British Columbia, Canada. \\ ${ }^{2}$ Department of Human Physiology, University of Oregon, Eugene, Oregon, USA.
}

Healthy adult aging is accompanied by decreases in muscle strength and power with reductions in standing balance control. Further, exerciseinduced muscle weakness in healthy young adults can lead to an increase in the vestibular control of balance. Yet, it remains unclear how the central nervous system compensates for a chronic decrease in muscle strength and power to maintain balance. The purpose was to determine whether vestibular control of standing balance is altered in older ( $n=8 ; 22.6 \pm 1.8$ years) compared to younger $(n=8 ; 69.7 \pm 6.7$ years) females and whether the vestibular-evoked balance response is related to muscle power. Participants stood quietly on a force plate, while subjected to random, continuous electrical vestibular stimulation (EVS; 0$20 \mathrm{~Hz}$, root mean square amplitude: $1.13 \mathrm{~mA}$ ). Medial gastrocnemius (MG) and tibialis anterior (TA) surface electromyography (EMG) and anterior-posterior forces $\left(\mathrm{F}_{\mathrm{AP}}\right)$ were sampled and quantified using frequency (coherence) and time (cumulant density) domain correlations with the EVS signal. Knee extensor strength and power were evaluated using a Biodex System 3 dynamometer. The weaker $(-34 \% ; p<0.05)$ and less powerful $(-39 \%$; $p<0.05)$ older females exhibited a greater medium-latency peak amplitude for the TA $(-99 \% ; p<0.05)$ and $\mathrm{F}_{\mathrm{AP}}(-42 \%$; $p<0.05$ ), but no other differences were detected for short- and mediumlatency peak amplitudes. The EVS-EMG coherence for the TA $(<10 \mathrm{~Hz})$ and MG $(<4 \mathrm{~Hz})$ and EVS-F $\mathrm{F}_{\mathrm{AP}}$ coherence $(<2 \mathrm{~Hz})$ were greater at lower frequencies in older females than young. A strong positive correlation was detected for $\mathrm{F}_{\mathrm{AP}}$ medium-latency peak amplitude with TA medium-latency peak amplitude $(r=0.86 ; p<0.05)$. Knee extensor power was negatively and moderately correlated with $\mathrm{F}_{\mathrm{AP}}$ medium-latency peak amplitude $(r=-0.47 ; p<0.05)$. These results suggest that the enhanced vestibular-evoked whole-body balance response (i.e., EVS-F $\mathrm{F}_{\mathrm{AP}}$ ) is related to muscle power capacity and greater TA activity. These results indicate that the increased vestibular control of balance may compensate for an age-related reduction in muscle power: 
Flow-mediate dilation is greater in upright posture in young healthy adults

\section{K.H. Habib and H. Edgell}

School of Kinesiology \& Health Sciences, Faculty of Health, York University, Toronto, ON M3J 1P3, Canada.

Orthostatic intolerance is often associated with vascular dysregulation leading to feelings of lightheadedness during upright posture. The baroreflex is activated during upright posture; however, intolerance can occur when there is an inadequate increase of peripheral vascular resistance. Hence, the purpose of this study was to assess vasodilatory capacity in the upright posture via measurements of brachial flow-mediated dilation (FMD) at the level of the heart. We hypothesized that healthy participants in the upright posture would experience significantly lower shear rate and FMD compared to the supine posture likely due to known increases of sympathetic activity. We recruited 10 female and 8 males $(20.6 \pm$ 1.8 years, $27.0 \pm 6.0 \mathrm{~kg} / \mathrm{m}^{2}$ ) with no history of cardiovascular or respiratory diseases. Women were tested in the low hormone phase of the menstrual cycle (days 2-5) and were not taking hormonal contraceptives. All participants completed 2 randomized FMD protocols ( 2 minutes baseline, 5 minutes right forearm occlusion, and 3 minutes recovery; 30-minute interval) in supine posture and $70^{\circ}$ head up tilt. FMD tests were both conducted with the brachial artery at the level of the heart to control for gravitational fluid shifts during upright posture. FMD was measured using duplex ultrasonography. Shear rate and percent FMD were calculated using automated software (Cardiovascular Suite). In the upright posture, participants exhibited a greater FMD response compared to the supine posture $(9.4 \pm 5.10 \%$ vs $14.8 \pm 3.82 \%, P=0.005)$. However, we observed no difference in shear rate between postures $\left(984 \pm 322 \mathrm{~s}^{-1}\right.$ vs $\left.940 \pm 272 \mathrm{~s}^{-1}, \mathrm{P} \geq 0.05\right)$. Furthermore, we observed no difference in the baseline diameter between postures $(3.5 \pm 0.79 \mathrm{~mm}$ vs $3.4 \pm 0.59 \mathrm{~mm}, \mathrm{P} \geq 0.05$ ). No interaction effects between posture and sex were present $(\mathrm{P} \geq 0.05)$. There is a greater FMD response in the upright posture compared to the supine posture, suggesting greater vasodilatory capacity in the upright posture. (Funding provided by the Natural Sciences and Engineering Research Council of Canada.)

A systematic review and meta-analysis of sex-based differences in resistance exercise training-induced changes in muscle mass, strength, and functional performance in healthy older $(\geq 60 \mathrm{y})$ adults

S.E. Hawley, ${ }^{1}$ Y. Huang, ${ }^{1}$ J.C. Gibbs, ${ }^{1,3}$ and T.A. Churchward-Venne ${ }^{1,2,3}$

${ }^{I}$ Department of Kinesiology and Physical Education, Mc Gill University, Montreal, QC H2W 1S4, Canada. ${ }^{2}$ Division of Geriatric Medicine, Mc Gill University, Montreal, QC H3G 1A4, Canada. ${ }^{3}$ Research Institute of the McGill University Health Centre, Montreal, QC H4A 3JI, Canada

Aging is associated with a reduction in muscle size, strength, and functional performance. These changes can be mitigated by resistance exercise training. It is unclear if there are sex-based differences in the adaptive response to resistance training in older adults. We performed a systematic review and meta-analysis of studies in healthy older ( $\geq 60$ y) males and females who performed identical resistance training interventions and had outcome measures of whole-body fat-free mass (FFM), skeletal muscle size, strength, and/ or functional performance. MEDLINE, EMBASE, CINAHL, SPORTDiscus, and Scopus were searched. We initially screened 1,323 titles/abstracts. Data from 37 studies (688 males and 787 females) were extracted. Mean study quality was moderate (17.8/28) on a modified Downs and Black checklist. Older males gained more absolute upper-body strength (Effect Size [ES] $=0.48$ [95\% CI: 0.16, 0.80], $p=0.004$ ), and absolute lower-body strength (ES $=0.28$ [95\% CI: 0.11, 0.45], $p=0.001$ ). Older females gained more relative lower-body strength $(\mathrm{ES}=-0.23$ [95\% CI: $-0.39,-0.07$ ], $p=0.006$ ), while relative gains in upper-body strength showed no sex differences. Older males gained more absolute wholebody FFM (ES: 0.22 [95\% CI: 0.08, 0.37], $p=0.003$ ), but there were no sex differences in relative gains in FFM. There were no significant sexbased differences in absolute or relative gains in type I, IIa, or IIx muscle fiber size or functional performance (i.e. chair rises and walking capacity tests). Our results indicate the existence of sex differences in the adaptive response to resistance training in healthy older adults. However, presentation of the results in either an absolute or relative format influences the sex-dependent differences in adaptations to resistance exercise training.

Assessment of cardiorespiratory fitness of young healthy adults after one year of COVID-19 restrictions

\section{E.T. Hedge and R.L. Hughson}

Schlegel-UW Research Institute for Aging, Waterloo, ON N2J OE2, Canada.

Government imposed restrictions to slow the spread of COVID-19 have unintentional health and fitness consequences due to limiting the physical activities that people can perform. We hypothesized that individuals would be less active during the COVID-19 pandemic leading to reduced cardiorespiratory fitness. Fourteen healthy adults ( 4 women, age: $27 \pm 6 \mathrm{yr}$ ) performed pseudorandom exercise between $25 \mathrm{~W}$ and $90 \%$ of ventilatory threshold and a $25 \mathrm{~W} \cdot \mathrm{min}^{-1}$ ramp exercise test to exhaustion prior to the pandemic and approximately one year later. Oxygen uptake $\left(\mathrm{VO}_{2}\right)$ was measured breath-by-breath. $\mathrm{VO}_{2}$ and heart rate kinetics were evaluated by the maximum cross-correlation function between the analyzed variable and work rate during pseudorandom exercise. Participants also completed the Global Physical Activity Questionnaire twice: once retrospectively to assess activity levels prior to restrictions and once to assess activity levels at the 1-year follow-up visit. Participants reported spending more time being sedentary each day (pre: $10.1 \pm 1.6$ vs. during: $11.7 \pm 2.4 \mathrm{hr} \cdot \mathrm{day}^{-1}$; $\mathrm{p}=0.014$ ) and less time being physically active each week (pre: $6.2 \pm 2.5$ vs. during: $4.1 \pm 3.6 \mathrm{hr} \cdot$ week $^{-1} ; \mathrm{p}=0.016$ ) during the pandemic. Absolute peak $\mathrm{VO}_{2}$ was not different between the pre- and during-pandemic time points (pre: $3.07 \pm 0.54$ vs. during: $2.93 \pm 0.42 \mathrm{~L} \cdot \mathrm{min}^{-1}$; $\mathrm{p}=0.072$ ), but did occur at a lower peak work rate (pre: $270 \pm 34$ vs. during: $260 \pm 30 \mathrm{~W} ; \mathrm{p}=0.022$ ) with a reduced peak $\mathrm{O}_{2}$ pulse (pre: $16.6 \pm 2.6$ vs. during: 15.6 \pm $2.1 \mathrm{~mL} \cdot$ beat $^{-1} ; \mathrm{p}=0.031$ ). Participants gained mass during the pandemic (pre: $72.7 \pm 11.1$ vs. during: $75.1 \pm 11.8 \mathrm{~kg} ; \mathrm{p}=0.007$ ) resulting in reduced relative peak $\mathrm{VO}_{2}$ (pre: $42.4 \pm 5.5$ vs. during: $39.4 \pm 5.2 \mathrm{~mL} \cdot \mathrm{min}^{-1} \cdot \mathrm{kg}^{-1}$; $\mathrm{p}=0.011)$. $\mathrm{VO}_{2}$ kinetics were not different between pre- and duringpandemic time points (pre: $0.67 \pm 0.04$ vs. during: $0.65 \pm 0.04$; $\mathrm{p}=0.102$ ), but heart rate kinetics were slower (pre: $0.70 \pm 0.04$ vs. during: $0.66 \pm$ $0.05 ; \mathrm{p}=0.005$ ). In support of our hypothesis, we provide evidence that physical activity patterns of young healthy adults were negatively impacted by the COVID-19 pandemic leading to a reduction in cardiorespiratory fitness. These results highlight the importance of finding alternative places and ways to exercise to maintain an active lifestyle during public health crises. (Supported by CIHR and NSERC.)

\section{Adaptations in serial sarcomere number and work loop performance to weighted downhill running in rats}

\section{A. Hinks, K. Jacob, P. Mashouri, and G.A. Power}

Department of Human Health and Nutritional Sciences, College of Biological Sciences, University of Guelph, Guelph, Ontario, Canada.

Optimal actin-myosin crossbridge formation occurs when sarcomeres are neither compressed nor overstretched. Forcing a muscle to act at an overstretched length can cause it to adapt via increased serial sarcomere number (SSN) to maintain optimal crossbridge overlap regions. Increased SSN has been observed in animals via downhill running training due to the emphasis on eccentric contractions, however, little is known about the influence of sarcomerogenesis on dynamic contractile function. We aimed to increase SSN in the rat 
soleus via 4 weeks of weighted downhill running training 3 days/week, then assess work loop performance in vitro at various levels of stretch and speed to characterize dynamic contractile function. We hypothesized that trained rats would perform better than controls in faster and more stretched work loops due to a greater SSN. 21 Sprague-Dawley rats were separated into a control $(n=14)$ or training group $(n=7)$. During training, weight was added via a vest and progressed from 5-15\% body mass. Following sacrifice, the soleus was dissected and tied to a force transducer and length controller, then the optimal muscle length for active force production $\left(\mathrm{L}_{\mathrm{O}}\right)$ was determined. Muscles then performed work loops (active shortening followed by passive lengthening) about $\mathrm{L}_{\mathrm{O}}$ at strains of 1-7 mm and cycling frequencies of 1.5-3 Hz, from which we measured work of shortening, work of lengthening, and net work (shortening minus lengthening work). After mechanical testing, muscles were fixed in formalin at $\mathrm{L}_{\mathrm{O}}$ for 1 week, then digested in nitric acid. Fascicle lengths and sarcomere lengths were measured via ImageJ and laser diffraction, respectively, and used to calculate SSN. Sarcomere length $(+0.14 \mu \mathrm{m})$ and SSN $(+379)$ trended toward being greater, and FL was significantly greater $(+2 \mathrm{~mm}$, $\mathrm{P}<0.01)$ in trained than control rats, demonstrating training-induced longitudinal muscle growth. $\mathrm{L}_{\mathrm{O}}$ trended toward increasing with training $(+0.8 \mathrm{~mm})$ and work of shortening increased in the faster work loops ( 2 and $3 \mathrm{~Hz})$ at 5 -mm strains $(\mathrm{P}<0.05)$; however, no other work loop parameters significantly differed with training. These preliminary results indicate longitudinal muscle growth has some effect on stretch and speed-dependent dynamic contractile function. (Supported by NSERC.)

Examining the association between change in estimated cardiorespiratory fitness and mortality

\author{
S.A. Houle, ${ }^{1}$ X. Sui,${ }^{2}$ and R. Ross ${ }^{1,3}$ \\ ${ }^{1}$ School of Kinesiology and Health Studies, Queen's University, Kingston, ON K7L 3N6, Canada. \\ ${ }^{2}$ Department of Exercise Science, Arnold School of Public Health, University of South Carolina, Columbia, \\ SC 29208, USA. \\ ${ }^{3}$ School of Medicine, Division of Endocrinology and Metabolism, Queen's University, Kingston, \\ ON K7L 3N6, Canada
}

Cardiorespiratory fitness (CRF) is a strong independent predictor of morbidity and mortality. Improvements in directly measured CRF are associated with marked reductions in mortality risk independent of age, race, or biological sex. The purpose of this investigation was to determine whether change in CRF estimated using non-exercise equations (eCRF) is associated with cardiovascular disease and all-cause mortality risk. Asymptomatic men ( $n=11002,44.6 \pm 9.3$ years) from the Aerobics Centre Longitudinal Study underwent two comprehensive medical examinations between 1979 and 2002 with a follow-up of $5.8 \pm$ 5.0 years. Change in eCRF was calculated using two published equations: Nes et al. (2011; Med. Sci. Sports Exerc. 43(11):2024-2030) and Jackson et al. (2012; Am. J. Prev. Med. 43(5):512-519). Analyses revealed that baseline eCRF derived from either equation was associated with significant reductions in mortality risk after multivariate adjustment (5-12\% per 1 metabolic equivalent [MET] higher eCRF). However, with few exceptions, change in eCRF was not associated with mortality from CVD or all-causes. A 1 MET improvement in Jackson eCRF was associated with a 7\% reduction in all-cause mortality risk when unadjusted. However, the relationship did not remain significant after adjustment for baseline eCRF and age ( $p>0.10$ ). Whereas for Nes eCRF, each 1 MET improvement in eCRF was associated with a $10 \%$ reduction in all-cause mortality risk after adjustment for baseline eCRF and age $(\mathrm{p}<0.05)$, the association did not remain significant after further adjustment for exam year and time between measures $(\mathrm{p}>0.10)$. Change in eCRF adjusted for covariates was not associated with CVD or all-cause mortality risk. Whether change in non-exercise estimated change in CRF is useful in health care settings requires further investigation.

\section{Influence of 4 weeks of downhill running on the calcium sensitivity of rat single muscle fibres}

\section{E.F. Hubbard, A. Hinks, P. Mashouri, and G.A. Power}

Neuromechanical Performance Research Lab, Department of Human Health and Nutritional Sciences, University of Guelph, Guelph, ON NIG 2W1, Canada.

Alterations in $\mathrm{Ca}^{2+}$ sensitivity have been suggested as a possible mechanism leading to improvements in muscle function following eccentric training, however, little is known regarding the effects of eccentric training on $\mathrm{Ca}^{2+}$ sensitivity in single muscle fibres. The only study investigating the effects of eccentric training on $\mathrm{Ca}^{2+}$ handling of single muscle fibres was performed in a group of adjuvantinduced arthritis rats. In that study, following eccentric training, $\mathrm{Ca}^{2+}$ activated force recovered nearly to the level of healthy controls, however it was not reported whether $\mathrm{Ca}^{2+}$ sensitivity improved. Therefore, in the present study we aimed to identify whether $\mathrm{Ca}^{2+}$ sensitivity is altered in vastus intermedius single muscle fibres from eccentrically trained compared to untrained male Sprague-Dawley rats. The training group underwent a four-week eccentric training protocol consisting of weighted downhill treadmill running three days per week at a $15^{\circ}$ decline and $16 \mathrm{~m} / \mathrm{min}$ speed for 35 minutes per day in five-minute bouts. After sacrifice, standard $\mathrm{Ca}^{2+}$ sensitivity tests of isolated fibres were performed using $\left[\mathrm{Ca}^{2+}\right]$ ranging from 7.0-4.5. Preliminary data from 10 control fibres and 10 eccentrically trained fibres showed no significant difference in specific force at maximal $\mathrm{Ca}^{2+}$ activating solution between control $\left(149 \pm 39 \mathrm{mN} / \mathrm{mm}^{2}\right)$ and training $\left(110 \pm 31 \mathrm{mN} / \mathrm{mm}^{2}\right)$ group, and pCa50 was 6.1 for both groups. These initial findings indicate that specific force and $\mathrm{Ca}^{2+}$ sensitivity were not affected by eccentric training. Next steps include increasing the sample size and muscle fibre typing to identify any fibre type-dependent effects of eccentric training on $\mathrm{Ca}^{2+}$ sensitivity. Identifying whether $\mathrm{Ca}^{2+}$ sensitivity plays a role in eccentric training-induced improvements in muscle function will enhance our understanding of the effects of eccentric training and will help to identify possible applications for eccentric-based interventions. (Supported by NSERC)

Personal strategies to minimize the effects of air pollution exposure during exercise

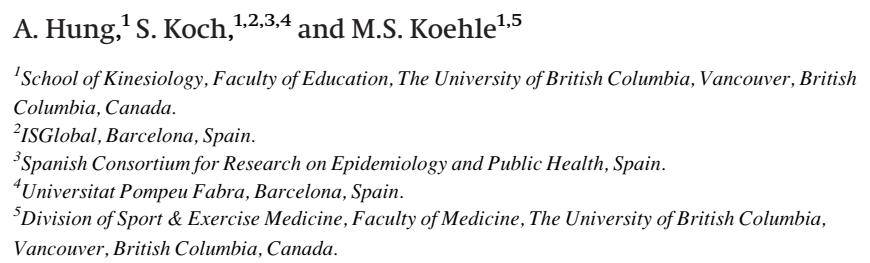

With global air pollution levels worsening over time, clinicians are increasingly being asked by their patients/athletes for guidance around exercising during periods of poor air quality. However, no clear guidelines exist on how to adjust physical activity levels in environments with high air pollution. The objective of this study was to review the existing literature on personal strategies to minimize the short-term effects of air pollution exposure during exercise and highlight practical strategies for clinicians to use when advising clients. MEDLINE, Embase, PubMed, CENTRAL, and SPORTDiscus databases were searched from inception to March 2021. Of the 1101 studies initially retrieved, 22 were included in the final review. Evidence suggests that personal exposure can be minimized by choosing low pollution environments (e.g., exercising away from major roadways), avoiding peak pollution periods (e.g., morning during the summer), and following local air quality forecasts (e.g., AQHI). When exercising in environments with high ozone levels, there is reasonable evidence for antioxidant supplementation (e.g., vitamin $\mathrm{C}$ or E). Limited data indicates a properly fitting high-efficiency particulate face mask (i.e., N95 respirator) in areas with high ambient levels of air pollution could improve surrogate markers of cardiorespiratory 
health. There was no evidence that asthma medications were protective nor detrimental to asthmatics and non-asthmatics exposed to ozone. A small number of studies suggest an ozone acclimatization protocol may be considered for athletes competing in environments with high levels of ozone pollution. It must be acknowledged the strategies are derived from a limited and uncertain evidence base. Well-designed studies in both healthy and vulnerable populations are warranted to provide strong, additional evidentiary support.

\section{Methodological reconciliation of CP and MLSS and their agreement with the maximal metabolic steady state}

D. Iannetta, ${ }^{1}$ C. Ingram, ${ }^{1}$ D.A. Keir, ${ }^{2}$ and J.M. Murias ${ }^{1}$

${ }^{I}$ Faculty of Kinesiology, University of Calgary, Calgary, AB, Canada

${ }^{2}$ School of Kinesiology, The University of Western Ontario, London, ON, Canada.

The critical power (CP) and maximal lactate steady state (MLSS) are operational surrogates of the maximal metabolic steady state (MMSS). However, their relationship and their agreement with MMSS remains variable likely due to inconsistent methods by which each may be determined. This study aimed to compare the correspondence between CP and MLSS determined by various models and criteria, respectively, and their agreement with MMSS. Ten recreationally-active males performed 4-5 severe-intensity constantpower output $(\mathrm{PO})$ trials to estimate $\mathrm{CP}$, and 3-4 constant-PO trials (at $10 \mathrm{~W}$ intervals) to determine MLSS and identify MMSS. CP was computed using the following models: 3-parameter hyperbolic $\left(\mathrm{CP}_{3 \text {-hyp }}\right)$ model and 2-parameter hyperbolic $\left(\mathrm{CP}_{2 \text {-hyp }}\right)$, linear $\left(\mathrm{CP}_{\text {lin }}\right)$, and inverse of time $\left(\mathrm{CP}_{1 / \text { Tlim }}\right)$ models from which the "best-fit' $\mathrm{CP}$ was also derived $\left(\mathrm{CP}_{\text {best-fit }}\right)$. MLSS was determined as an increase in blood lactate concentration $\leq 1 \mathrm{mM}$ during constant-PO cycling from the $5^{\text {th }}\left(\mathrm{MLSS}_{5-30}\right), 10^{\text {th }}\left(\mathrm{MLSS}_{10-30}\right), 15^{\text {th }}\left(\mathrm{MLSS}_{15-30}\right), 20^{\text {th }}\left(\mathrm{MLSS}_{20-30}\right)$, or $25^{\text {th }}$ $\left(\right.$ MLSS $\left._{25-30}\right)$ to $30^{\text {th }}$ minute. MMSS was identified as the highest PO associated with submaximal, steady state $\dot{\mathrm{VO}}_{2}$. At the group level, none of the CP and MLSS estimates were different from each other $(P>0.05)$. However, the PO corresponding to MLSS $_{5-30}$ was lower than the POs corresponding to $\mathrm{MLSS}_{15-30}$, MLSS ${ }_{20-30}$, and $\mathrm{MLSS}_{25-30}(P<0.05)$. With respect to the model-estimates from the three, 2-parameter $\mathrm{CP}$ models, concordance (CCC) was poor when considering MLSS $_{5-30}$ and MLSS $_{10-30}$ (aggregate CCC: 0.85 and 0.89 , respectively) but moderate-to-substantial when considering $\mathrm{MLSS}_{15-30}, \mathrm{MLSS}_{20-30}$, and $\mathrm{MLSS}_{25-30}$ (aggregate CCC: 0.94, 0.94, 0.96, respectively). Of the CP estimates, $\mathrm{CP}_{2 \text {-hyp }}(244 \pm 46 \mathrm{~W})$ and $\mathrm{CP}_{\text {lin }}(248 \pm 46 \mathrm{~W})$ displayed the greatest agreement with MMSS. Of the MLSS criteria, MLSS $15-30$ and MLSS $_{20-30}$ (both 245 $\pm 46 \mathrm{~W}$ ) were in closest agreement with MMSS. Estimates of CP and MLSS can be reconciled by refining methods of MLSS determination. MLSS $_{15-30}$ and MLSS $_{20-30}$ demonstrated the lowest probability of error. (Supported by NSERC and HSFC.)

Sex- and fitness-related differences in microvascular responses to reactive hyperemia

\section{E.C. Inglis, ${ }^{1}$ L. Rasica, ${ }^{1}$ D. Iannetta, ${ }^{1}$ R.N. Soares, ${ }^{2}$ and J.M. Murias ${ }^{1}$ \\ ${ }^{I}$ Faculty of Kinesiology, University of Calgary, Calgary, AB T2N 1N4, Canada. \\ ${ }^{2}$ Dalton Cardiovascular Research Center, University of Missouri, Columbia, MO 65203, USA}

Recently, the use of near-infrared spectroscopy (NIRS) combined with a vascular occlusion test (NIRS-VOT) has been shown to be a reliable technique for the assessment of skeletal muscle microvascular responsiveness. Importantly, NIRS-VOT-derived reperfusion parameters have been shown to be sensitive enough to detect difference in microvascular responsiveness in populations with both impaired (clinical) and enhanced (physically active) vascular health. However, it is unknown whether sex-related differences in microvascular responsiveness exist in both chronically trained (CT) and untrained (UT) populations. Fifteen CT ( 8 males, 7 females) and sixteen UT ( 8 males, 8 females) participants were tested. NIRS-VOT assessments were performed on vastus lateralis of the right leg including: a 2-min baseline, followed by a 5-min limb occlusion period, proceeded by a 5-min reperfusion assessment. Maximal oxygen uptake $\left(\mathrm{V} \mathrm{O}_{2 \max }\right)$ was measured during a cycling incremental exercise test to volitional exhaustion. A faster reperfusion rate was found in CT vs UT ( $F=7.01 ; p=0.013)$, as well as in males vs females $(F=9.41 ; p=0.005)$. Peak muscle oxygen saturation $\left(\mathrm{StO}_{2 \text { peak }}\right)$ was greater in UT vs CT participants $(F=8.51 ; p=0.007)$ and lower values were detected in the muscle oxygen saturation amplitude $\left(\mathrm{StO}_{2 \mathrm{amp}}\right)$ for females vs males $(F=8.17 ; p=0.008)$. $\dot{\mathrm{O}}_{2 \max }$ was significantly correlated with $\mathrm{StO}_{2}$ reperfusion rate $\left(r^{2}=0.347 ; r=0.589 ; p=0.0005\right)$. The results of this study demonstrate that, at the microvascular level, there are sex- and fitness-related differences in reperfusion rates as faster reperfusion rates were found in CT vs UT and males vs females. These differences may be related to functional and morphological differences that exist in these participants. Further investigation is needed to elucidate the underlying mechanisms. (Supported by NSERC and the Heart and Stroke Foundation of Canada.)

\section{The reliability of residual force enhancement in vivo}

\section{K. Jacob, A. Hinks, and G.A. Power}

Department of Human Health and Nutritional Sciences, College of Biological Sciences, University of Guelph, 50 Stone Road East, Guelph, Ontario, Canada.

Residual force enhancement ( $\mathrm{rFE}$ ) is a property of muscle characterized by increased steady-state isometric force following active muscle lengthening as compared to a purely isometric (ISO) contraction at the same muscle length and level of activation. Previous studies have characterized rFE on a dynamometer across multiple visits, including longitudinal training studies; however, the reliability has not been assessed. Here we investigated day-to-day reliability of rFE on a HUMAC NORM dynamometer. Five young adults (3F, 2M) completed 2 visits separated by 1 week. In each visit, maximum voluntary contraction (MVC) strength was assessed, and the interpolated twitch technique confirmed $>95 \%$ voluntary activation before continuing with the experimental procedures. Both voluntary $(100 \%$ MVC), and electrically stimulated contractions (20\%MVC) were used to assess $\mathrm{rFE}$, at short $\left(0^{\circ}\right.$ to $20^{\circ}$ plantar flexion $\left.(\mathrm{PF})\right)$ and long $\left(0^{\circ}\right.$ to $\left.40^{\circ} \mathrm{PF}\right)$ muscle-tendon unit lengths. For the voluntary contractions, participants first performed a 10-s MVC, then $2 \mathrm{rFE}$ contractions. rFE contractions consisted of a 2-s isometric pre-activation phase at $0^{\circ} \mathrm{PF}$ followed by an isokinetic $\left(20^{\circ} / \mathrm{s}\right)$ lengthening $\left(0^{\circ}\right.$ to $20^{\circ}$ or $\left.40^{\circ} \mathrm{PF}\right)$ and ending with a 6-s steady state isometric phase at $20^{\circ}$ or $40^{\circ} \mathrm{PF}$. For the electrically stimulated contractions, electrodes were placed on the tibialis anterior and stimulated at $50 \mathrm{~Hz}$ to reach the $20 \% \mathrm{MVC}$ target and held for 10-s, then the same current was used for rFE contractions. The rFE contractions with the highest torque between each set of 2 were chosen for analysis. Voluntary and electrically stimulated contractions at both muscle lengths and both visits showed significant $\operatorname{rFE}(\mathrm{p}<0.05)$, ranging from $7-29 \%$. The highest reliability for voluntary contractions (ICC:0.75-0.86) appeared at the shorter muscle length, while the highest reliability for electrically stimulated (ICC:0.808-0.894) appeared at the longer muscle length. Therefore, based on the preliminary data, reliability of these history-dependent contractions appears to be muscle length dependent. (Supported by NSERC)

\section{The effect of exercise-induced muscle weakness on isometric performance fatigability of the dorsiflexors in males and females}

\section{H.L. Jodoin, V.S. Contento, and G.A. Power}

Department of Human Health and Nutritional Sciences, College of Biological Sciences, University of Guelph, Guelph, Ontario, Canada.

Performance fatigability can be defined as any exercise-induced transient impairment in the ability to generate force or power regardless of whether the task can be sustained, and is highly task-, 
and population-dependent. Across most studies, females are more fatigue resistant than males during sustained isometric contractions. This sex-difference is most prominent at lower isometric contraction intensities, and becomes more variable during higher intensity isometric, and dynamic contractions. While less fatiguing than isometric or concentric contractions, high intensity eccentric contractions induce greater and longer lasting impairment of force production (muscle weakness). However, it is not clear how muscle weakness influences susceptibility to performance fatigability in males and females during sustained isometric contractions. The purpose of this study was to investigate the effects of exerciseinduced muscle weakness via high-intensity eccentric contractions on time to task failure (TTF) of the dorsiflexors during a sustained submaximal isometric fatigue task in males and females. Using a HUMAC NORM dynamometer, seven females (22 4 yr) and six males $(23 \pm 3 \mathrm{yr})$ performed a dorsiflexion isometric fatigue task at $35^{\circ}$ plantar flexion by matching a $30 \%$ submaximal torque target, until task failure. Task failure was defined as falling below $5 \%$ of the target torque for $\geq 2 \mathrm{~s}$. The same isometric fatigue task was repeated 30 minutes after 5 sets of 30 maximal eccentric contractions. At baseline, males were $\sim 45 \%$ stronger than females despite no difference in voluntary activation. At this point in data collection, there was no significant sex-difference in TTF, however we expect to see the typical sex-differences in fatigability. Following the unaccustomed eccentric contractions, males had a $\sim 44 \%$ reduction in strength and females had a $\sim 22 \%$ reduction. Following exerciseinduced weakness, TTF which was reduced similarly for both males ( 75\%) and females ( 57\%). (Supported by NSERC)

\section{The impact of aerobic exercise on indices of cardiac function and exercise tolerance in females with breast cancer receiving anthracycline-based chemotherapy: An interim report from the EXACT2.0 clinical trial}

W.J. Johnston, ${ }^{2}$ S. Varghese, ${ }^{1}$ C. Eekhoudt, ${ }^{1}$ D. Cheung, ${ }^{1}$ C. Barnes, ${ }^{1}$ T. Bortoluzzi, ${ }^{1}$ H. Bews,${ }^{1}$ M. McKenzie, ${ }^{1}$ I. Mittal, ${ }^{1}$ U. Ismail ${ }^{1}$ J. Chiekwe, ${ }^{2}$ M. Keats, ${ }^{2}$ T. Nguyen, ${ }^{1}$ M. Pitz, ${ }^{1}$ S.L. Mulvagh, ${ }^{2}$ D.S. Jassal, and S.A. Grandy ${ }^{2}$

${ }^{1}$ University of Manitoba, Winnipeg, Canada.

${ }^{2}$ Dalhousie University and Nova Scotia Health Authority, Halifax, Canada.

The cardioprotective effects of aerobic exercise (AE) in mitigating anthracycline (AC)-induced cardiotoxicity in females with breast cancer (BC) remain unclear. We investigated the impact of a 24-week homebased $\mathrm{AE}$ program on cardiac function and exercise tolerance in females with $\mathrm{BC}$ receiving AC. Participants (52 \pm 10 years-old) were randomized to control [standard of care (SOC); $\mathrm{n}=7$ ] or SOC+24-week home-based AE program $(\mathrm{n}=8)$. The exercise program consisted of two self-directed sessions per week (35-85\% incremental heart rate reserve) to achieve 90-minutes of exercise weekly. Cardiac function [left ventricular ejection fraction (LVEF) and global longitudinal strain (GLS)], was assessed using serial transthoracic echocardiography at baseline and at 24-weeks. Total time exercising during the Bruce treadmill exercise stress test performed at baseline and 24-week follow-up was the proxy measure for exercise tolerance. At baseline, mean exercise time was $408.60 \pm 23.62 \mathrm{~s}$ and $402.17 \pm 42.14 \mathrm{~s}$ in the control and $\mathrm{AE}$ group, respectively. After 24 -weeks, the exercise time was $439.00 \pm 65.59 \mathrm{~s}$ and $462.00 \pm 71.34 \mathrm{~s}$ respectively in the control and $\mathrm{AE}$ groups, respectively ( $\mathrm{p}=0.650$ ). At baseline, the LVEF was $62 \pm 2 \%$ and $63 \pm 2 \%$ in the control and AE groups, respectively. After 24-weeks, the LVEF was $62 \pm 3 \%$ and $58 \pm 8 \%$ in the control and exercise groups, respectively $(\mathrm{p}=0.250)$. At baseline, the mean GLS was $-19.5 \pm 1.5 \%$ and $-18.7 \pm$ $1.2 \%$ in the control and AE groups, respectively. At 24-weeks, mean GLS was $-18.2 \pm 1.3 \%$ and $-17.5 \pm 2.3 \%$ in the control and AE groups, respectively $(\mathrm{p}=0.658)$. These preliminary findings indicate that a 24-week home-based AE program did not significantly improve exercise tolerance or cardiac function in females with $\mathrm{BC}$ receiving AC.
Senescence-associated metabolomic profiling of skeletal muscle cells

M.J. Kamal, ${ }^{1}$ S. Joanisse, ${ }^{2}$ P. Britz-McKibbin, ${ }^{3}$ and G. Parise ${ }^{1}$

${ }^{\prime}$ Department of Kinesiology, McMaster University, Hamilton, ON L8S 4L8, Canada.

${ }^{2}$ Department of Sport and Exercise Sciences, Manchester Metropolitan University, Manchester, United Kingdom ${ }^{3}$ Department of Chemistry and Chemical Biology, McMaster University, Hamilton, ON L8S 4L8, Canada.

Cellular senescence is a widespread molecular response that occurs due to DNA damage and is common in aging tissues and cells. Following a fixed number of divisions or irreparable genomic damage, most healthy cells become senescent. Senescent cells secrete factors capable of in vivo reprogramming of adjacent cells. Collectively, these factors are known as the senescence-associated secretory phenotype (SASP). Prior research has examined the involvement of cellular senescence in a variety of aging-related diseases, however deciphering the role of senescence and the SASP in aging muscle remains understudied. Therefore, the purpose of this study was two-fold: 1) develop an inducible senescence model in skeletal muscle cells and 2) define the metabolome associated with induced senescence. C2C12 myoblasts were treated with either a chemotherapy drug, bleomycin (Bleo), to induce DNA damage, or a vehicle control. Myoblasts were examined 24-hours following treatment for senescence-related biomarkers, such as proliferation status, cell viability, senescence-associated $\beta$-galactosidase (SA $\beta$-Gal) activity, gene expression of $\mathrm{p} 21$ and $\mathrm{p} 53$, and $\gamma \mathrm{H} 2 \mathrm{AX}$ protein content. Furthermore, metabolomic profiling using capillary electrophoresis - mass spectrometry (CE-MS) was performed on cells and their media 24-hours following treatment. Bleo treatment was highly effective at inducing cellular senescence in myoblasts as evidenced by stark alterations in cell morphology and a $50 \%$ reduction in proliferation $(\mathrm{p}<0.05)$, with no impact on cell viability. Bleo-treated myoblasts also displayed canonical senescence indicators including 3-fold greater SA $\beta$-Gal activity, elevated p21 and p53 gene expression, and greater $\gamma \mathrm{H} 2 \mathrm{AX}$ protein content $(\mathrm{p}<0.05)$. CE-MS analysis revealed that media from senescent cells contained 2- to 3-fold greater concentration of metabotoxins that have been previously linked to endothelial cell senescence and neurological aging $(\mathrm{p}<0.05)$. Future work will aim to evaluate the direct impact of these metabolites on myoblasts to help further characterize the impact of senescence and the SASP on skeletal muscle.

\section{Effects of lung volume on signal-averaged sympathetic transduction of blood pressure}

C. Katerberg, ${ }^{1}$ M. Nardone, ${ }^{1}$ A.L. Teixeira, ${ }^{1}$ A.V. Incognito, ${ }^{1}$ T.D. Vermeulen, ${ }^{2}$ B.M. Shafer, ${ }^{2}$ P.J. Millar, ${ }^{1,3}$ and G.E. Foster ${ }^{2}$

${ }^{I}$ Department of Human Health and Nutritional Sciences, University of Guelph, Guelph, ON, Canada ${ }^{2}$ Centre for Heart, Lung, and Vascular Health, School of Health and Exercise Sciences, The University of British Columbia, Kelowna, BC, Canada.

${ }^{3}$ Toronto General Research Institute, Toronto General Hospital, Toronto, ON, Canada.

Muscle sympathetic nerve activity (MSNA) is known to exhibit within-breath respiratory modulation. The present study set out to assess the effect of lung volume on sympathetic transduction of blood pressure. Seventeen healthy young males underwent an 810 min baseline collection in a semi-recumbent position of continuous measures of blood pressure, heart rate, MSNA, ventilation, and end-tidal gases. Sympathetic transduction was quantified using burst-triggered signal averaging such that serial changes in mean arterial pressure (MAP), cardiac output (CO), total vascular conductance (TVC), heart rate (HR), stroke volume (SV), and diastolic blood pressure (DBP) were calculated across 15 cardiac cycles. Cardiac cycles were grouped into low vs. high lung volumes based on the simultaneous lung volume corresponding to each R-wave of the electrocardiogram. Low lung volume was defined by volumes less than the median inspiratory and expiratory volumes across all cardiac cycles, while high lung volumes were defined by the opposite. Sympathetic transduction was then calculated. Sympathetic transduction of DBP was larger during low compared with high lung volumes $(\Delta 2.7 \pm 1.2 \mathrm{mmHg}$ vs. $\Delta 1.3 \pm 1.2 \mathrm{mmHg}, P<0.01)$. These 
pressure responses are influenced strongly by within-breath changes in $\mathrm{HR}$ and SV; at low lung volumes, HR increased $(\Delta 1.4 \pm 1.8$ beats $/ \mathrm{min})$ and SV decreased $(\Delta-0.1 \pm 2.4 \mathrm{~mL})$. At high volumes, HR decreased $(\Delta-3.7 \pm 1.8$ beats $/ \mathrm{min})$ and SV increased $(\Delta 7.4 \pm 2.3 \mathrm{~mL})$. Given that TVC was similar across low $(\Delta-0.1 \pm 2.1 \mathrm{~mL} / \mathrm{min} / \mathrm{mmHg})$ and high lung volumes $(\Delta-1.0 \pm 1.9 \mathrm{~mL} / \mathrm{min} / \mathrm{mmHg})$, the differences in the pressor response to an MSNA burst is not the result of altered vasoconstriction. In conclusion, non-neural factors, such as respiration, are likely to play a role in signal-averaged sympathetic transduction of blood pressure through modulation of HR and SV.

\section{The Effects of a Single Bout of Isometric Handgrip Exercise on} Nighttime Blood Pressure in Young Normotensive Men and Women

Muhammad Kathia, ${ }^{1}$ Matthias Purvis, ${ }^{1}$ and Philip Millar ${ }^{1,2}$

${ }^{\prime}$ Department of Human Health and Nutritional Sciences, University of Guelph, Guelph, ON NIG 2W6, Canada ${ }^{2}$ Toronto General Research Institute, Toronto General Hospital, Toronto, ON M5G 2C4, Canada.

Nighttime blood pressure (BP) is a better predictor of adverse cardiovascular outcomes and all-cause mortality than clinical BP measurements. Although participating in regular isometric handgrip (IHG) exercise is a time-efficient method of reducing resting BP, whether a single bout of IHG exercise acutely reduces nighttime and ambulatory BP due to post-exercise hypotension is unknown. We measured 24-hr ambulatory BP in 16 normotensives $(11$ men and 5 women, age $=23 \pm 0.5$ years, BMI $=26.61 \pm 4.95 \mathrm{~kg} / \mathrm{m}^{2}$ ) following either control or acute IHG visits performed in a randomized crossover design. The participants performed four 2-minute unilateral isometric contractions ( 2 contractions on each side) at $30 \%$ of their maximum voluntary contraction (MVC) with a minute of rest between each contraction before bedtime during their IHG visit, whereas no such exercise was performed during the control visit. No significant differences were observed in the nighttime dip of systolic BP (SBP) $(-18.5 \pm 6.4$ vs. $-16.6 \pm 6.2 \Delta \mathrm{mmHg}$, IHG vs. control, mean $\pm \mathrm{SD}, \mathrm{p}=0.36)$, diastolic $\mathrm{BP}(\mathrm{DBP})(-17.2 \pm 6.1 \mathrm{vs}$. $-16.2 \pm 3.9 \Delta \mathrm{mmHg}, \mathrm{p}=0.32$ ) or mean arterial pressure (MAP) $(-17.7 \pm 5.7$ vs. $-16.5 \pm 3.9 \Delta \mathrm{mmHg}, \mathrm{p}=0.28)$. There were trends towards a lower 24-hour SBP (115.9 \pm 12.5 vs. $114 \pm 11.2 \mathrm{mmHg}, \mathrm{p}=0.06)$ and 16-hours post-IHG SBP $(113.3 \pm 14.1$ vs. $110.9 \pm 12.0, \mathrm{p}=0.08)$ during the IHG visit, but not in the DBP and MAP of 24-hour and 16-hour post-IHG when compared to the control visit (all $\mathrm{p}>0.05$ ). In conclusion, an acute bout of evening IHG exercise did not cause a significant reduction in the nighttime BP dip, though there was evidence for a potential reduction in 24-hour and 16-hour post-IHG SBP.

\section{The Effects of Exercise on Anthracyline Induced Cardiotoxicity in Breast Cancer Survivors}

S.J. Kendall, ${ }^{1}$ C.J. Chiekwe, ${ }^{1}$ W.J. Johnston, ${ }^{1,2}$ M. Keats, ${ }^{1,2}$ D.R. Bouchard, ${ }^{3,4}$ C. Soucy, ${ }^{3,4}$ and S.A. Grandy ${ }^{1,2}$

${ }^{I}$ School of Health and Human Performance, Dalhousie University, Halifax, NS, Canada.

${ }^{2}$ Beatrice Hunter Cancer research Institute, Halifax, NS, Canada.

${ }^{3}$ Faculty of Kinesiology, University of New Brunswick, Fredericton NB, Canada.

${ }^{4}$ Cardiometabolic Exercise \& Lifestyle Laboratory, University of New Brunswick, Fredericton, NB, Canada.

Anthracyclines (ACs) are a chemotherapeutic drug used to treat breast cancer. Cardiotoxicity is a long-term side-effect of AC treatment that can lead to left ventricular dysfunction and heart failure. Currently, there is no gold standard treatment for AC-induced cardiotoxicity. However, the research suggests that exercise may be an effective treatment to mitigate the damaging effects. Therefore, the purpose of this study was to determine if a 12-week multi-mode exercise intervention (e.g. aerobic, resistance, functional) improves heart health outcomes in cancer survivors (CS) treated with ACs. A retrospective analysis was conducted based upon data collected from community exercise programs in Halifax NS and Fredericton NB. A total of 34 female breast CS were included in the analysis $\left(\mu_{\text {age }}=\right.$
$58.2 \pm 10.0$ ). CS were divided into two groups based on whether they had received $\mathrm{AC}(\mathrm{n}=26)$ or not $(\mathrm{n}=8)$. Heart health was assessed pre- and post-intervention using the 6-minute walk test. Specifically, walk distance was used to predict $\mathrm{VO}_{2}$ peak, an indicator of heart health, using the equation $\mathrm{VO}_{2}$ peak $=0.03 \mathrm{x}$ distance $(\mathrm{m})+3.98$. The change in $\mathrm{V}_{2}$ peak data was compared between baseline and post-exercise intervention. The results indicate that a 12 -week multi-mode exercise program improved $\dot{V}_{2}$ peak over the course of the intervention in females both receiving and not receiving ACs $(\mathrm{p}<0.001)$. Furthermore, an ANOVA indicated that receiving ACs does not significantly impact the CS's ability to improve their $\mathrm{VO}_{2}$ peak $(\mathrm{p}=0.72)$. Therefore, it appears that a multimodal community exercise program can improve the heart health in breast CS independently of AC usage.

Muscle-strengthening activities influence body fat in both women and men living with obesity: Is sex influencing the association between MSA and health outcomes?

M. Keshavarz, ${ }^{1,2}$ M. Sénéchal, ${ }^{1,2}$ and D.R. Bouchard ${ }^{1,2}$

${ }^{I}$ Cardiometabolic Exercise \& Lifestyle Lab, University of New Brunswick, Fredericton, NB Canada.

${ }^{2}$ Faculty of Kinesiology, University of New Brunswick, Fredericton, NB Canada.

While muscle-strengthening activities (MSA) two or more times per week are recommended for both men and women living with obesity, it is unknown whether sex has different impacts on health outcomes and mortality. To describe the proportion of adults living with obesity doing MSA (2X/week) by sex and obesity levels; and test if the associations between performing MSA (2X/week), body fat percentage (BF\%) and mortality are influenced by sex. The data from 4202 adults living with obesity (BF $\geq 25 \%$ men and $\geq 35 \%$ women) from the National Health and Nutrition Examination Survey (NHANES 2003-04, 2005-06) were analyzed. MSA (2X/week) was self-reported via a questionnaire. BF \% was estimated via Dual Energy X-ray and mortality obtained via administrative data for an average of 10 years post-testing. BF\% and mortality were predicted using weighted regression models adjusting for aerobic activities (accelerometer), sex, MSA, sex*MSA, age, and the sum of five categories of self-reported chronic conditions (arthritis, cardiovascular diseases, diabetes, lung diseases and cancers). Body mass index (BMI) was calculated and used to create obesity levels. The proportion of men and women living with obesity performing MSA (2X/week) was $18.7 \%$ and $15.2 \%$, respectively $(P=0.03)$. A significant difference between men and women was observed among two BMI groups (25-29.9) ( $\mathrm{p} \leq 0.001)$ and (35-39.9) ( $\mathrm{p} \leq 0.001)$. Performing MSA (2X/week) was significantly associated with a lower $\mathrm{BF} \%(\mathrm{P}=0.004)$, but not mortality $(\mathrm{P}=0.32)$. The interaction between sex and MSA (2X/week) was not significantly associated with the outcomes. Men living with obesity do more MSA compared with their women counterparts. Performing MSA (2X/week) is associated with greater BF\% but not mortality. Based on the findings, MSA does not impact men and women differently for the studied outcomes.

Training status is related to lactate stability at the maximal lactate steady state after mild elevation in carboxyhemoglobin

\section{H. Kontro, C. Bertagnolli, J.M. Murias, and M.J. MacInnis}

Human Performance Lab, Faculty of Kinesiology, University of Calgary, Calgary, AB T2N 1N4, Canada.

Convective oxygen delivery as a determinant of the maximal lactate steady state (MLSS) has not been investigated. This study sought to evaluate the effect of an elevated carboxyhemoglobin $(\mathrm{COHb})$ content, consequential to hemoglobin mass evaluation, on the stability of the [La] response at the predetermined MLSS. 16 volunteers (8 female) undertook two trials in a randomized, double-blind design, after performing the carbon monoxide (CO) rebreathing procedure or a sham procedure (SHAM). Compared to SHAM, COHb was significantly higher 
before $(5.9[0.5] \%$ vs. $0.9[0.1] \%)$ and after (3.7 [0.5]\% vs. $0.8[0.1] \%)$ the 30-min constant load test in the CO condition ( $\mathrm{p}<0.001$ for both). The $[\mathrm{La}](\mathrm{p}=0.004)$, ventilation $(\mathrm{p}=0.010)$, heart rate $(\mathrm{p}=0.007)$, and RPE $(\mathrm{p}=0.047)$ were increased by $\mathrm{CO}$, while $\dot{\mathrm{VO}}_{2}(\mathrm{p}=0.136)$ and $\dot{\mathrm{V}}_{2}$ $(\mathrm{p}=0.128)$ were unaffected by CO. Low-frequency fatigue of the quadriceps was greater in CO than SHAM $(-30[18] \%$ vs. $-23[14] \%, \mathrm{p}=0.023)$, as assessed by femoral nerve electrical stimulation before and after exercise. Some participants $(\mathrm{n}=7)$ had a stable [La] between 10 and $30 \mathrm{~min}$ in the $\mathrm{CO}$ condition while others $(\mathrm{n}=9)$ did not. When these two groups were compared, the unstable group had a higher $\mathrm{VO}_{2} \max$ (65.7 [8.5] vs. $\left.55.8[8.6] \mathrm{mL} \cdot \mathrm{kg} \mathrm{LBM}^{-1} \cdot \mathrm{min}^{-1}, \mathrm{p}=0.036\right)$, higher MLSS (55.2 [6.8] vs. 43.8 [6.6] $\left.\mathrm{mL} \cdot \mathrm{kg} \mathrm{LBM}^{-1} \cdot \mathrm{min}^{-1}, \mathrm{p}=0.004\right)$, higher $\mathrm{RCP}$ as \% of $\mathrm{VO}_{2} \mathrm{max}$ ( $88[5] \%$ vs. $82[4] \%, \mathrm{P}=0.012$ ), and a smaller drift of $\mathrm{VO}_{2}$ between 10 and 30 min in sham MLSS (2.4 [1.7] \% vs. $4.8[1.4] \%, \mathrm{p}=0.009)$. In summary, the capacity to stabilize [La] at MLSS with elevated $\mathrm{COHb}$ appears to be related to aerobic fitness status, indicating that oxygen delivery may be more limiting to this threshold in trained individuals. (Funded by the Natural Sciences and Engineering Research Council of Canada)

\section{Sclerostin regulates exercise induced adaptations in energy} expenditure and body composition in $\mathrm{C57BL} / 6 \mathrm{~J} \mathrm{mice}$

N. Kurgan, ${ }^{1,2}$ J. Stoikos, ${ }^{1,2}$ B.J. Baranowski, ${ }^{3}$ R. Dhaliwal, ${ }^{3}$ J.B. Sweezey-Munroe, ${ }^{3}$ V.A. Fajardo, ${ }^{1,2}$ R.E.K. MacPherson, ${ }^{3,4}$ and P. Klentrou ${ }^{1,2}$

${ }^{I}$ Department of Kinesiology, Brock University, St. Catharines, ON L2S 3A1, Canada. ${ }^{2}$ Centre for Bone and Muscle Health, Brock University, St. Catharines, ON L2S 3A1, Canada ${ }^{3}$ Department of Health Sciences, Brock University, St. Catharines, ON L2S 3A1, Canada. ${ }^{4}$ Centre for Neuroscience, Brock University, St. Catharines, ON L2S 3A1, Canada.

Produced by osteocytes, sclerostin is an inhibitor of the osteogenic Wnt/B-catenin signaling pathway and has an endocrine role in regulating adipose tissue (AT) by reducing adipogenesis. We have previously shown that subcutaneous white AT (scWAT) sclerostin content transiently increases immediately following acute exercise but decreases at rest following 4 weeks of exercise training (EXT) in both humans and mice. Therefore, we hypothesized that EXT induced reductions in adipose tissue sclerostin may play a role in regulating adaptations in body composition and whole-body metabolism. To test this hypothesis, 10-week-old male C57BL/6J mice were either sedentary (SED) or performing $1 \mathrm{~h}$ of treadmill running at $\sim 65 \%-70 \% \mathrm{~V}_{2 \max } 5 \mathrm{~d} /$ week (EXT) for 4 weeks and had s.c injections of either saline (C) or a physiologically relevant dose of recombinant sclerostin (S) $(0.1 \mathrm{mg} / \mathrm{kg}$ body mass) $5 \mathrm{~d}$ /week; thus, making 4 groups (SED-C, EXT-C, SED-S, and EXT-S; n= 12/group). No differences in body mass were observed between experimental groups, however food intake was higher in EXT $(\mathrm{p}=0.03)$ and $\mathrm{S}$ $(\mathrm{p}=0.08)$ groups. This is in agreement with a higher resting energy expenditure in each of the treatment groups compared to SED-C. EXT-C had a higher lean mass and lower \%fat mass compared to SED-C and SED-S. No differences in body composition were observed in either the SED-S or EXT-S groups. Reductions in scWAT and vWAT mass were observed in the EXT-C group and a reduction in vWAT was observed in the EXT-S group, demonstrating differences in adipose tissue mass with EXT and S treatments. Together these results suggest that sclerostin may play a role in exercise induced alterations in energy metabolism and body composition. This study was funded by NSERC.

\section{A Pilot Study Investigating Problematic Exercise Behavior Patterns Amongst Varsity Level University Athletes}

\section{E. Landolfi}

School of Kinesiology, University of the Fraser Valley, Abbotsford, BC V2S 7M8, Canada.

The purpose of the study was to examine the prevalence of exercise dependence amongst varsity-level university athletes. Physical activity behavior patterns were measured amongst 62 participants (31 male and 31 female) from a mid-size University in Canada for the purpose of identifying problematic exercise through a questionnaire-based survey utilizing the Exercise Addiction Inventory (EAI). Total scores from the EAI questionnaires were tabulated to indicate the number of varsity athletes who scored either "at-risk", "symptomatic" or "asymptomatic" of exercise addiction, and percentages were determined for each of the EAI categories. Results indicate that $72.5 \%$ of the sample scored "symptomatic" while $19.5 \%$ recorded "at-risk" and $8 \%$ attained a score of "asymptomatic" on the EAI questionnaire. Interestingly, three times as many female athletes rated "at-risk" of engaging in problematic exercise compared to their male athlete counterparts, whereas males outnumbered females at a ratio of four to one with respect to scoring "asymptomatic" on the Exercise Addiction Inventory scale. People should be encouraged to embrace activity so that it enhances their life through sustainable long-term physical, psychological and social health versus exercising for deriving a sense of physical and psychological fulfillment. In addition, underlying causes such as anxiety, depression, difficulties with relationships or coping with personal hardship can be at the root of exercise addiction. Consequently, individuals who score in the "at-risk" category of the EAI might be best advised to seek counselling.

\section{Children's adherence to 24-hour movement guidelines during the second wave of COVID-19: national survey}

R. Larouche, ${ }^{1}$ M. Bélanger, ${ }^{2}$ M. Brussoni, ${ }^{3}$ G. Faulkner, ${ }^{4}$ K. Gunnell, ${ }^{5}$ and M.S. Tremblay ${ }^{6}$

${ }^{I}$ Faculty of Health Sciences, University of Lethbridge, Lethbridge, AB TIK 3M4, Canada.

${ }^{2}$ Faculté de médecine et des sciences de la santé, Université de Sherbrooke, Sherbrooke, QC J1H 5N4, Canada.

${ }^{3}$ Department of Pediatrics, The University of British Columbia, Vancouver, BC V6H 3V4, Canada.

${ }^{4}$ School of Kinesiology, The University of British Columbia, Vancouver, BC V6T IN1, Canada.

${ }^{5}$ Department of Psychology, Carleton University, Ottawa, ON K1S 5B6, Canada.

${ }^{6}$ Healthy Active Living and Obesity Research Group, CHEO Research Institute, Ottawa, ON K1H 8L1, Canada.

A survey conducted during the first wave of COVID-19 found that only $4.8 \%$ of Canadian children met the 24-hour movement guidelines. Given the evolving public health restrictions, we examined adherence to the guidelines during the second wave of the pandemic (December 2020) and disparities by sociodemographic characteristics. We employed baseline data from a national longitudinal survey conducted online with 2,291 parents of 7- to 12-year-olds (48.5\% girls). The survey included questions about children's moderate- to vigorous-intensity physical activity (MVPA), screen time, sleep, and sociodemographic variables. We computed the proportion of children meeting the 24-hour guidelines and each individual guideline. We used binary logistic regression models to examine how adherence to 24-hour guidelines varied by gender, age, region, household income, and how children attended school in the preceding week (i.e., in person vs. others [e.g., online, blended]). Parentreported adherence to individual guidelines was $69.6 \%$ for MVPA, $22.5 \%$ for screen time, and $54.9 \%$ for sleep. Overall, $9.9 \%$ of children met the three guidelines (8.9\% of boys and $11.1 \%$ of girls; $p=0.136$ ). Controlling for gender, odds of meeting the guidelines decreased with each year of age ( $\mathrm{OR}=0.71 ; 95 \% \mathrm{CI}=0.64-0.79$ ) and were lower in children living in Ontario vs. the Atlantic provinces $(\mathrm{OR}=0.51 ; 0.28-0.93)$, in families earning $\$ 40,000-99,999$ vs. $\geq \$ 100,000$ (OR=0.69; 0.48-1.00) and in children who did not attend school in person $(\mathrm{OR}=0.30 ; 95 \% \mathrm{CI}=0.17-0.52)$. Findings suggest a need for promoting healthy movement behaviours and addressing inequalities in a pandemic context. (Supported by HSFC).

\section{Sex differences in muscle metaboreflex activation following static handgrip exercise}

Jordan B. Lee, ${ }^{1}$ Karam Notay, ${ }_{1}^{1}$ Jeremy D. Seed, ${ }^{1}$ Lucas J. Omazic, ${ }^{1}$ Massimo Nardone, ${ }^{1}$ and Philip J. Millar ${ }^{1,2}$

${ }^{I}$ Department of Human Health and Nutritional Sciences, University of Guelph, Guelph, ON N1G 2W6, Canada ${ }^{2}$ Toronto General Research Institute, Toronto General Hospital, Toronto, ON M5G 2C4, Canada.

Males have larger blood pressure (BP) responses than females during matched relative intensity static handgrip (HG) exercise. This is 
thought to be due to greater metaboreflex activation in males as evidenced by larger BP responses during post-exercise circulatory occlusion (PECO). We have shown that controlling for differences in maximal voluntary contraction (MVC) or absolute contraction intensity ameliorates sex differences in BP during static HG exercise. Alternatively, BP responses may differ between males and females due to an intrinsic sex difference in metabolite production or transduction of sympathetic outflow, independent of absolute workload. Thus, we aimed to determine the predictors of the BP response to PECO in a large cohort of males and females. Continuous BP and heart rate were recorded in 200 young healthy males and females ( $\mathrm{n}=109$ females) during 2 minutes of static HG at 30\% of MVC, followed by 2 minutes of PECO. Muscle sympathetic nerve activity (MSNA) was collected in a subset of participants ( $n=39$; female $n=21$ ) and used to calculate signal-averaged sympathetic transduction. Sex differences in hemodynamic and neural responses were examined with and without statistical adjustment for MVC. Multivariate regression analyses were performed to determine predictors of the $\mathrm{BP}$ response. Systolic BP responses were greater in males (interactions, $P<0.0001)$ during static HG exercise $(24 \pm 10 \mathrm{vs.} 17 \pm 9 \mathrm{mmHg}$ [mean $\pm S D], P<0.0001)$ and PECO $(20 \pm 11$ vs. $16 \pm 9 \mathrm{mmHg}, P<0.0001)$. These differences were abolished when adjusted for MVC (interactions, $P>0.7)$. MSNA responses to HG and PECO were similar between males and females (interactions, $P>0.2$ ), as was resting sympathetic transduction $(P=0.8)$. MVC and the change in MSNA were significant predictors of the systolic BP response to static HG, while MVC was the only predictor associated with systolic BP responses during PECO. In conclusion, sex differences in metaboreflex activation are related to differences in absolute contraction intensity, rather than an intrinsic effect of sex per se. (Supported by NSERC.)

Evaluating the relationships between bone-specific physical activity and nutrition behaviours and measures of bone mineral density in young men and women

J. Levee, A. Sevinc, X. Qi, N. Fripp, L. Tsoukalas, J. Chemtov, R. Andersen, and J.C. Gibbs

Department of Kinesiology and Physical Education, McGill University, Montreal, QC H2W 1S4, Canada.

High-impact physical activity and adequate nutrition represent important health behaviours in optimizing musculoskeletal health. However, few studies have evaluated the gender-specific differences in these behavioural determinants of bone mineral density (BMD). Our study compared the relationships between bone-specific physical activity and nutrition behaviours and measures of BMD in young men and women. We recruited healthy, young adults aged 18-35 years for one-time measures. Bone-specific and moderate-tovigorous physical activity (MVPA) levels were self-reported using the Bone-specific Physical Activity Questionnaire (BPAQ) and International Physical Activity Questionnaire. Calcium intake was determined using a calcium food frequency questionnaire. BMD at the proximal femur and lumbar spine and body composition were measured by dual energy X-ray absorptiometry. Independent t-tests were used to compare outcomes by gender. Pearson correlation analyses adjusting for gender were performed. Twenty-three participants were included in this preliminary analysis $(57 \%$ women, mean \pm SD age $21.7 \pm 1.8$ years, BMI $23.1 \pm 2.7 \mathrm{~kg} / \mathrm{m}^{2}$, body fat $24.4 \pm$ $7.8 \%$, MVPA $381 \pm 248 \mathrm{~min} /$ week). There were no gender differences in BPAQ scores and calcium intake $(\mathrm{p}>0.05)$. However, men demonstrated higher femoral neck $\left(1.22 \pm 0.09\right.$ vs. $\left.1.02 \pm 0.09 \mathrm{~g} / \mathrm{cm}^{2}, \mathrm{p}=0.002\right)$ and total hip BMD $\left(1.20 \pm 0.09\right.$ vs. $\left.1.06 \pm 0.14 \mathrm{~g} / \mathrm{cm}^{2}, \mathrm{p}=0.016\right)$ than women. Past and total BPAQ scores were positively correlated with femoral neck and total hip BMD $(\mathrm{r}=0.425-0.502, \mathrm{p}<0.05)$. However, there were no statistically significant associations between BPAQ scores, calcium intake, and any other BMD variables $(\mathrm{p}>0.05)$. After adjusting for gender, correlations remained significant between past
BPAQ score and femoral neck $(\mathrm{r}=0.499, \mathrm{p}=0.03)$ and total hip BMD $(\mathrm{r}=0.484, \mathrm{p}=0.036)$, and between total BPAQ score and femoral neck BMD ( $\mathrm{r}=0.471, \mathrm{p}=0.042)$. In conclusion, bone-specific physical activity was positively associated with BMD at the femoral neck and total hip regardless of gender. This research provides new knowledge in understanding the behavioural determinants of bone health in young adults. (Supported by McGill University Start-Up Grant Funding.)

\section{Does aerobic fitness impact prolonged sitting-induced popliteal} artery endothelial dysfunction?

\section{H. Liu, M.W. O’Brien, J.A. Johns, and D.S. Kimmerly}

Autonomic Cardiovascular Control and Exercise Lab, Division of Kinesiology, School of Health and Human Performance, Faculty of Health, Dalhousie University, Halifax, NS, Canada.

Acute prolonged bouts of sitting reduce popliteal artery blood flow and flow-mediated dilation (FMD). Individuals with higher aerobic fitness have enhanced popliteal FMD. Conflicting evidence regarding whether more aerobically fit individuals are protected from the negative impacts of sitting on popliteal endothelial function in maledominated studies have been reported. We further explored the relationship between aerobic fitness and sitting-induced impairments in popliteal blood flow and FMD in a more sex-balanced cohort. Relative peak oxygen consumption $\left(\mathrm{VO}_{2}\right.$ peak) was assessed using a cycling-based incremental test in 21 healthy adults ( 8 males; $23 \pm 2$ years; $23.9 \pm$ $2.9 \mathrm{~kg} / \mathrm{m}^{2}$ ). Popliteal blood flow and relative FMD (\%) were measured via duplex ultrasonography before and after 3-h of uninterrupted sitting. Pearson correlations were performed separately between $\mathrm{VO}_{2}$ peak versus Pre-sitting and sitting-induced reductions in popliteal outcomes. Aerobic fitness $(41.0 \pm 9.7 \mathrm{~mL} / \mathrm{kg} / \mathrm{min})$ was positively correlated with Pre-sitting popliteal blood flow $(65 \pm 23 \mathrm{~mL} / \mathrm{min} ; \mathrm{R}=0.59, \mathrm{P}=0.005)$ and relative FMD $(4.2 \pm 1.5 \%$; $\mathrm{R}=0.49, \mathrm{P}=0.03)$. As expected, sitting reduced resting blood flow $(19 \pm 11 \mathrm{~mL} / \mathrm{min})$ and FMD $(1.9 \pm 0.7 \%)$ (both, $\mathrm{P}<0.001$ ). $\mathrm{VO}_{2}$ peak was inversely related to sitting-induced declines in blood flow $(\Delta-46 \pm 23 \mathrm{~mL} / \mathrm{min} ; \mathrm{R}=-0.71, \mathrm{P}<0.001)$ and FMD $(\Delta-2.4 \pm+1.5 \%$; $R=-0.51, P=0.02)$. Although higher aerobic fitness was associated with more favorable popliteal endothelialdependent vasodilator responses, it also corresponded with larger sitting-induced impairments in FMD. This suggests that being more aerobically fit does not protect against sitting-induced vascular endothelial dysfunction. As such, all young adults should minimize habitual prolonged sedentary bouts, regardless of their aerobic fitness level.

\section{Investigating physical activity intensity of virtual reality exergame in recreationally active young adults}

\section{A.A. Lorenz, C.P. Quinn, C.R. van Rassel, M. Shin, and R.J. Holash}

Faculty of Kinesiology, University of Calgary, AB T2N 1N4, Canada.

The purpose of this study is to determine the relative intensity of physical-activity from playing the virtual reality game Beat Saber ${ }^{\circledR}$. Male $(n=6)$ and female $(n=4)$ subjects (ages 18-27 years) completed two laboratory visits: 1) In the first visit, subjects completed a graded exercise test to determine the gas exchange threshold (GET), respiratory compensation point (RCP), $\dot{\mathrm{VO}}_{2 \max }$ and $\mathrm{HR}_{\max }$ 2) In the second visit, subjects played Beat Saber ${ }^{\circledR}$ while ventilatory parameters were measured. Relative intensity for each subject achieved during gameplay was reported as $\mathrm{VO}_{2}$ expressed as a percentage of GET and RCP, metabolic equivalents (METs), rating of perceived exertion (RPE), and percent heart rate max (\%HRmax) Additionally, average values for $\mathrm{VO}_{2}$ during gameplay, $\mathrm{V}_{2}$ at GET, and $\dot{\mathrm{VO}}_{2}$ at RCP were calculated as a percentage of $\dot{\mathrm{VO}}_{2 \max }$ for individuals and represented as $\% \dot{\mathrm{VO}}_{2 \mathrm{VR}}, \% \dot{\mathrm{VO}}_{2 \mathrm{GET}}$, and \% $\dot{\mathrm{VO}}_{2 \mathrm{GET}}$, respectively. Using the Canadian Society for Exercise Physiology (CSEP) 
guidelines and game play intensity quantified with METs, RPE, and $\% \mathrm{HR}_{\max }$, it was determined that Beat Saber ${ }^{\circledR}$ elicits moderate physical activity, in most subjects. When we individualised subjects' $\% \mathrm{VO}_{2 \mathrm{VR}}$ with a their GET and RCP values, this also indicated that playing Beat Saber ${ }^{\circledR}$ elicits moderate intensity physical activity. In conclusion, Beat Saber ${ }^{\circledR}$ can be used to meet physical activities guidelines in some players as defined by CSEP. However, in novice players, Beat Saber ${ }^{\circledR}$ may not be an appropriate mode of exercise to meet CSEP recommendations for the minimum amount of moderate to vigorous physical activity in a healthy adult population. (This study's funding was by provided by an internal start up grant from the faculty of Kinesiology)

\section{Outcome domains measured in randomized controlled trials of} physical activity for older adults: A rapid review

D.C. Mackey, ${ }^{1}$ C.L. Ekegren, ${ }^{2}$ A. Ciok, ${ }^{1}$ and A. Wong ${ }^{1}$

${ }^{I}$ Department of Biomedical Physiology and Kinesiology, Simon Fraser University, Burnaby, BC V5A 1S6, Canada.

${ }^{2}$ School of Primary and Allied Health Care, Monash University, Frankston, VIC 3199 Australia.

We conducted a rapid review to identify outcome domains ("what" was measured) in randomized controlled trials (RCTs) of physical activity for older adults. We searched Ovid MEDLINE for recentlypublished (2015-2021), English-language, RCTs of physical activity interventions for older adults (mean age 60+ yrs). We limited to articles published in top-10 journals in general and internal medicine, geriatrics and gerontology, rehabilitation, and sports science by Web of Science impact factor. Our search yielded 548 articles; two reviewers agreed to exclude 466 during citation screening and 15 during full-text screening, resulting in 67 included articles from which two reviewers abstracted trial descriptors and study outcomes. We classified study outcomes according to the standard outcome classification taxonomy endorsed by the Core Outcome Measures in Effectiveness Trials Initiative. Of the 67 articles, 52\% reported on RCTs conducted in North America, $85 \%$ included participants of both sexes, and $84 \%$ recruited community-dwelling older adults. In addition, $40 \%$ of articles reported on interventions that involved a combination of group and individual physical activities, and $60 \%$ involved a combination of physical activity modes (e.g., aerobic, resistance, balance). We identified 21 unique outcome domains, spanning 4 core areas (physiological/clinical, life impact, resource use, adverse events); no outcome domains were reported in all RCTs. The five most commonly reported outcome domains were physical functioning (included in $\mathrm{n}=54$ articles articles), general $(n=22)$, injury and poisoning $(n=18)$, cognitive functioning $(\mathrm{n}=16)$, and emotional functioning/wellbeing $(\mathrm{n}=11)$. This rapid review found extensive variability in outcome domains used in RCTs of physical activity for older adults, reflecting the broad range of health benefits derived from physical activity. This work will inform development of a core outcome set to improve outcome reporting consistency and evidence quality (Supported by MSFHR, and The Drummond Foundation).

Estimation of the $\mathrm{VO}_{2}$ mean response time utilizing two step transitions of different amplitudes preceding a ramp-incremental test

M. Mackie, ${ }^{1}$ D. Iannetta, ${ }^{1}$ R.J. Holash, ${ }^{1}$ D.A. Keir, ${ }^{2}$ and J.M. Murias ${ }^{1}$

${ }^{I}$ Faculty of Kinesiology, University of Calgary, Calgary, AB T2N 1N4, Canada.

${ }^{2}$ School of Kinesiology, The University of Western Ontario, London, ON, Canada.

Accurate estimation of the mean response time (MRT) of oxygen uptake $\left(\mathrm{VO}_{2}\right)$ following a ramp incremental (RI) test to exhaustion is critical for appropriately time-aligning the $\mathrm{VO}_{2}$-to-power output (PO) relationship. By performing a step transition within the moderate intensity domain (MOD), preceding the RI test, the constant- load $\mathrm{VO}_{2}$ at a given $\mathrm{PO}$ can optimize the reliability of the MRT. However, the PO associated with MOD is often unknown before the RI test. This preliminary study compared the estimated MRT when performing two step transitions of different intensities, one of which represented virtually the lowest increase in PO where changes in $\dot{\mathrm{VO}}_{2}$ can be confidently evaluated. Ten healthy individuals (4 females, 6 males) performed a RI cycling test $\left(30 \mathrm{~W} \cdot \mathrm{min}^{-1}\right)$ to exhaustion preceded by two step transitions from a $20-\mathrm{W}$ to $50 \mathrm{~W}$ and $80 \mathrm{~W}$. The steady state $\mathrm{VO}_{2}$ from both the $50 \mathrm{~W}$ and $80 \mathrm{~W}$ MODs was extrapolated to the ramp test and the POs associated to those $\mathrm{VO}_{2}$ values during the RI test were calculated. The difference between the RI PO with both MODs was converted into time (s) to compute the MRT. Paired T-Test and B\&A analysis were used to compare the MRT from each MOD. The MRT from the $50 \mathrm{~W}$ MOD $(19.2 \pm 19.1 \mathrm{~s})$ and the $80 \mathrm{~W}$ MOD $(20.4 \pm 13.0 \mathrm{~s})$ were not significantly different $(\mathrm{p}=0.78)$, with a bias not different from zero $(-1.2 \mathrm{~s} ; \mathrm{z}=-0.30)$, and upper and lower limits of agreement (LoA) corresponding to $24.7 \mathrm{~s}$ and $-27.1 \mathrm{~s}$, respectively. In conclusion, despite the not significant differences in the mean values for the MRT and a bias not different from zero, the wide LoA suggest a larger than expected variability when the MRT is estimated from lower compared to larger step transitions within the MOD. (Funding by NSERC and HSFC.)

\section{Ventilatory response of peripheral chemosensors to hypercapnia} during exercise

L.M. Mann, ${ }^{1}$ J.S. Chan, ${ }^{1}$ S.A. Angus, ${ }^{1}$ B. Thompson, ${ }^{1}$ C.J. Doherty, ${ }^{1}$ G.E. Foster, ${ }^{2}$ R.L. Hughson, ${ }^{1}$ and P.B. Dominelli ${ }^{1}$

${ }^{I}$ Department of Kinesiology, University of Waterloo, Waterloo, ON N2L 3G1, Canada. ${ }^{2}$ School of Health and Exercise Science, The University of British Columbia, Kelowna, BC VIV IV7, Canada.

Hypercapnic chemosensitivity is the ventilatory response to increased partial pressure of $\mathrm{CO}_{2}$ and is the result of central and peripheral chemosensors stimulation. The response of the chemosensors has been examined at rest and related to the ventilatory response during exercise; however, the sensitivity of the peripheral chemoreceptors during exercise is less understood. We sought to examine the peripheral chemoreceptors' contribution to hypercapnic sensitivity during moderate intensity exercise in individuals of varying fitness. We hypothesized that athletically trained individuals will have a reduced ventilatory response compared to those who are untrained. Twenty-five healthy subjects ( $\mathrm{n}=11$ females) participated in one test day involving transient hypercapnic chemosensitivity testing and a maximal exercise test. Chemosensitivity testing involved two breaths of $10 \% \mathrm{CO}_{2}$ repeated five times (30-45 sec between repeats) at rest and during the first two stages of exercise. Stage- 1 of exercise started at $60 \mathrm{~W}$ and $80 \mathrm{~W}$ for females and males respectively, and both increased by $20 \mathrm{~W}$ for stage-2. After stage-2, intensity increased in $20 \mathrm{~W}$ increments every 1.5 minutes for both sexes. The subjects were divided into high or low fitness based on the median $\mathrm{VO}_{2 \max }$ for each sex. The high fitness group had a greater maximal ventilation $(151 \pm 43 \mathrm{vs} .120 \pm 30 \mathrm{~L} / \mathrm{min}, \mathrm{p}<0.05)$ however, the metabolic equivalents $\left(\dot{\mathrm{V}}_{\mathrm{E}} \dot{\mathrm{N}} \mathrm{CO}_{2}, \dot{\mathrm{V}}_{\mathrm{E}} \dot{\mathrm{N}} \mathrm{O}_{2}\right)$ were not different $(\mathrm{p}>0.05)$. There was no main effect of fitness on average chemosensitivity $(\mathrm{p}=0.185)$ nor an interaction between fitness and exercise intensity ( $\mathrm{p}=0.581)$. There was also no significant relationship between $\dot{\mathrm{V}}_{2 \max }$ and hypercapnic chemosensitivity response at any stage $(\mathrm{R}=0.371)$. There was a significant effect of intensity whereby the hypercapnic ventilatory response was greater for stage-1 of exercise $(1.081 \pm 0.47 \mathrm{~L} / \mathrm{min} \cdot \mathrm{mmHg})$ compared to rest $(0.786 \pm$ $0.5 \mathrm{~L} / \mathrm{min} \cdot \mathrm{mmHg})(\mathrm{p}<0.05)$; however, there were no difference to stage-2 $(0.94 \pm 0.42 \mathrm{~L} / \mathrm{min} \cdot \mathrm{mmHg})$. We conclude that aerobic fitness does not impact the hypercapnic ventilatory response. (Supported by NSERC) 
The impact of abdominal body contouring surgery on physical function after a massive weight loss: a pilot non-randomized controlled trial

S. Mardan-Dezfouli, ${ }^{1}$ M.D. Fliss, ${ }^{1}$ O.G. Reid, ${ }^{2,4}$ D. Harris, ${ }^{4}$ S. Sampath,,${ }^{3,4}$ N. Nguyen, ${ }^{3,4}$ E. Yorke, ${ }^{3,4}$ M.R. Lyon, ${ }^{5}$ and C.J. Mitchell ${ }^{1}$

${ }^{I}$ School of Kinesiology, Faculty of Education, The University of British Columbia, Vancouver, BC, Canada ${ }^{2}$ Faculty of Medicine, Department of Plastic Surgery, The University of British Columbia, Vancouver, BC, Canada.

${ }^{3}$ Faculty of Medicine, Department of Surgery, The University of British Columbia, Vancouver, BC, Canada ${ }^{4}$ Richmond Metabolic and Bariatric Surgery, Richmond, BC, Canada

${ }_{5}^{5}$ Obesity Medicine and Diabetes Institute, Coquitlam, BC, Canada.

Approximately $70 \%$ of individuals develop excess skin (ES) following massive weight loss (MWL). The abdominal ES negatively impacts physical function in studies of patient reported outcomes. Yet the impact of abdominal body contouring surgeries (ABCS) on direct measures of physical function is unknown. The objective of this pilot study was to determine the variability and reliability of a battery of physical performance tests in adults following MWL. Additionally, the effect sizes of ABCS on physical performance measures were estimated to appropriately power future trials. Twenty-four patients $(47.2 \pm 9.2 \mathrm{y})$ who have undergone MWL of $40.0 \pm 8.5 \%$, had Body Mass Index (BMI) of $29.0 \pm 4.2 \mathrm{~kg} / \mathrm{m}^{2}$ and had grade 2-3 ES were self selected as control (no surgery $n=20$ ) or planned to undergo ABCS ( $\mathrm{n}=4$ ). Participants (92\% females) completed the 9-item modified physical performance test (mPPT), 30schair to stand, star excursion balance test (SEBT), timed up and go, modified agility t-test, and 6-minute walk test (6-MWT) at baseline and 8-12 weeks later to accommodate recovery time from surgery (ABCS group). Retention (72\%) and recruitment (46\%) rates were compromised due to Covid-19 pandemic related local restrictions, and institutional surgical regulations. No adverse events were observed. No ceiling or floor effect was detected for any physical function measure. Intra-class correlation (ICC) with $95 \%$ confidence revealed good to excellent reliability for most physical performance measures although a $0.5 \pm 0$.9-point learning effect was detected for the MPPT. The effect sizes with $75 \%$ confidence were adequate for SEBT $0.74(0.03,0.10)$, Modified agility t-test $-1.16(-1.82$, $-0.50)$, and 6-MWT 0.65 (0.01,1.29). Tests involving dynamic balance, agility, and walking were reliable and showed medium to large effect sizes suggesting that agility and balance are likely improved by ABCS in those with grade 2-3 ES. In order to archive $80 \%$ power, future clinical trials should include between 12 (agility) and 29 (balance) participants per group. To reach sufficient trial enrolment it is recommended that future studies recruit from at least four high volume surgical practices and conduct physical function testing in at least two geographically separate facilities. ClinicalTrials.gov Identifier: NCT04516473, The University of British Columbia internal funding.

\section{The heart rate at the respiratory compensation point is not a valid surrogate to derive the heart rate at the maximal metabolic steady state during endurance exercise}

\section{G. Marinari, J.M. Murias, and D. Iannetta}

Faculty of Kinesiology, University of Calgary, Calgary, Canada

The $\mathrm{VO}_{2}$ at the respiratory compensation point (RCP) has been repeatedly shown to be closely related to the $\mathrm{V}_{2}$ associated to the maximal metabolic steady state (MMSS), the boundary separating the heavy- from the severe-intensity domains. On the basis of power output (PO), however, the correspondence of RCP and MMSS is more variable because the $\mathrm{PO}$ at the RCP is protocol dependent. This equivocality limits the use of the $\mathrm{PO}$ at the RCP for exercise intensity prescription. Considering that $\mathrm{VO}_{2}$ and heart rate (HR) response are tightly related, and that the $\mathrm{VO}_{2}$ at the RCP and MMSS are similar, in this study we investigated whether the HR at RCP would correspond to the HR at MMSS. Eleven male adults performed one ramp-test $\left(30 \mathrm{~W} \cdot \mathrm{min}^{-1}\right)$, and two-to-three 30 -min constant-PO trials for MMSS determination. Thereafter, the HR at the RCP was computed by averaging a 20-s window around the $\mathrm{VO}_{2}$ at RCP, and the HR at MMSS was computed by averaging a 60 -s window around the $20^{\text {th }}$ min (reflecting the beginning of the steady state $\mathrm{VO}_{2}$ ). Results confirmed that $\mathrm{VO}_{2}$ at RCP $\left(2.86 \pm 0.61 \mathrm{~L} \cdot \mathrm{min}^{-1}\right)$ was not different and strongly correlated with a small mean bias to the $\mathrm{VO}_{2}$ at MMSS $\left(2.90 \pm 0.67 \mathrm{~L} \cdot \mathrm{min}^{-1}\right)(P>0.05 ; \mathrm{r}=0.99$; bias $=0.03, \mathrm{z}=-1.12$, limits of agreement (LoA) -0.24 to $\left.0.18 \mathrm{~L} \cdot \mathrm{min}^{-1}\right)$. Although HR at RCP $(162 \pm$ $8 \mathrm{bpm})$ was not different from the HR at MMSS $(166 \pm 7 \mathrm{bpm})(P>0.05)$, the mean bias between the HR at RCP and at MMSS was significant $(-6 \mathrm{bpm})(\mathrm{z}=-2.34$, LoA: -23 to $11 \mathrm{bpm})$ and the measures were not correlated $(\mathrm{r}=0.263)$. In conclusion, despite RCP and MMSS occur at the same $\dot{\mathrm{VO}}_{2}$, their corresponding $\mathrm{HR}$ showed poor concordance. Although the physiological mechanisms for this dissociation remains unclear, these findings indicate that the HR at the ramp-derived RCP is not a valid surrogate measure to estimate MMSS. (Supported by NSERC and HSFC.)

\section{The effects of a gradual VCD-induced ovarian failure model of perimenopause on single fibre muscle contractility in mice}

P. Mashouri, ${ }^{1}$ J. Saboune, ${ }^{2}$ W.G. Pyle, ${ }^{2,3}$ and G. Power ${ }^{1}$

${ }^{I}$ Neuromechanical Performance Research Lab, Human Health and Nutritional Sciences, University of Guelph, Guelph, ON N1G 2W1, Canada.

${ }^{2}$ Laboratory of Molecular Cardiology, Biomedical Sciences, University of Guelph, Guelph, N1G2W1, Canada. ${ }^{3}$ IMPART Investigator Team, Dalhousie Medicine, Saint John, New Brunswick, E2K 5E2, Canada

Throughout menopause, estrogen deficiency is associated with a loss of muscle mass and strength. In ovariectomized (OVX) rodent models, estrogen deficiency impairs muscle contractile performance owing to altered contractile protein function, but not changes to $\mathrm{Ca}^{2+}$ sensitivity. While OVX models quickly reduce circulating sex-hormones, a model with greater ecological validity and gradual decrease in physiological levels of circulating estrogens is warranted. Here we investigated $\mathrm{Ca}^{2+}$ sensitivity and cross-bridge kinetics of fast- and slow-type fibres of the EDL and soleus muscle, respectively, in mice under-going ovarian failure. Perimenopause was induced in sexually mature CD1 female mice by injecting 4-vinylcyclohexene diepoxide (VCD) to cause gradual ovarian failure over 120 days. Mice showed acyclicity in estrus cycles by day 120 indicating the end of perimenopause and the start of the menopause phase. Single muscle fibres were dissected from the EDL and soleus, and chemically permeabilized. A single muscle fibre was mounted between a force transducer and length controller, and activated across varying $\mathrm{Ca}^{2+}$ concentrations (pCa 4.5-7.0). Preliminary data from control (4 fast and 9 slow fibres) and VCD mice (7 fast and 11 slow fibres) indicates a $30 \%$ reduction in absolute force for fast-type fibres, and a $10 \%$ reduction in normalized force (force/CSA) for slow-type fibres in VCD versus control mice. There was no interaction between group $\times$ muscle for $\mathrm{Ca}^{2+}$ sensitivity. There were no differences in $\mathrm{Ca}^{2+}$ sensitivity across groups for both fast and slow type muscle, however absolute and normalized force was affected in a fibre-type dependent manner. Analysis of cross-bridge kinetics (instantaneous active stiffness and the rate of transition from non-force bearing to force bearing cross-bridge states) are ongoing to identify potential mechanisms of impaired muscle contractile function in perimenopause. (Supported by NSERC and HSFC.)

Effect of increased dairy consumption on bone markers and osteokines during one-week of intense training in adolescent female athletes

K.M. McKee, B.J. McKinlay, N. Kurgan, B. Roy, B. Falk, and N. Klentrou

Department of Kinesiology, Faculty of Applied Health Sciences, Brock University. St. Catharines, ON L2S 3A1, Canada.

While high-impact exercise training typically has a positive effect on bone, intensified training during adolescence, the period of rapid growth and peak bone acquisition, could potentially have 
an opposite effect. Dairy foods contain bone-supporting nutrients (e.g., calcium) that are crucial to the structural integrity and strength of bone. In this study, 13 female adolescent soccer players $(14.3 \pm 1.3 y)$ participated in a cross-over, randomized, double-blind trial examining the effects of Greek yogurt (GY) consumption on bone biomarkers during a 5-d period of intensified training. The study took place over two intervention weeks, which consisted of a pre-training assessment day, 5-days of consecutive, intense soccer training and a post-training assessment day. Participants completed both the GY condition, and a carbohydrate isocaloric placebo control $(\mathrm{CHO})$ condition in random order, 4 weeks apart. Fasted, blood samples were collected in the morning pre- and post-training sessions during each intervention condition. Total osteocalcin (tOC), undercarboxylated osteocalcin (unOC), c-terminal telopeptide of type 1 collagen (CTX), osteoprotegerin (OPG), and receptor activator nuclear factor kappa- $\beta$ ligand (RANKL) were measured in serum. Results showed no significant effects for time (from pre- to posttraining) nor condition, and no interaction in tOC, CTX, OPG, RANKL and OPG/RANKL ratio. There was a significant interaction $(\mathrm{p}=0.011)$ for unOC. Pairwise comparisons revealed that unOC decreased significantly at the end of the intense training period in the GY condition, but not in the CHO condition $(-26 \%$ vs. $-3 \%$, respectively). Relative unOC, expressed as a percentage of tOC, also decreased post-training $(-16 \%)$, but with no differences between intervention conditions. These findings suggest that high-impact intense training had no direct catabolic impact on bone metabolism, at least in the short-term, and thus, GY added no benefit beyond that of the isocaloric $\mathrm{CHO}$ control. (The study was funded by the Natural Sciences and Engineering Research Council of Canada NSERC and was registered at Clinicaltrials.gov (NCT03947801).)

\section{The long-term effect of incident fractures on self-reported physical activity in adults}

E.C. McLaughlin, ${ }^{1}$ L.M. Giangregorio, ${ }^{1,2}$ J.D. Adachi, ${ }^{3}$ A.C. Laing, ${ }^{1}$ A. Papaiannou, ${ }^{4}$ L. Thabane, ${ }^{5}$ and C. McArthur ${ }^{6}$

${ }^{I}$ Department of Kinesiology and Health Sciences, University of Waterloo, Waterloo, ON, Canada. ${ }^{2}$ Schlegel-UW Research Institute for Aging, Waterloo, ON, Canada.

${ }^{3}$ Department of Medicine, McMaster University, Hamilton, ON, Canada.

${ }^{4}$ GERAS Centre for Aging Research, Hamilton, ON, Canada.

${ }^{5}$ Department of Health Research Methods, Evidence, and Impact, McMaster University, Hamilton, ON, Canada.

${ }^{6}$ Dalhousie University, Halifax, NS, Canada.

Physical activity is decreased in the acute period following a fracture, and impairments in mobility and ambulation can persist with resulting disability. Therefore, a fracture may have long-term consequences on physical activity participation. We explored effects of incident hip or pelvic, spine, upper limb, and lower limb fracture on self-reported physical activity at five- and ten-year follow-up. 9423 community-dwelling adults aged $\geq 25$ years, participating in the Canadian Multicentre Osteoporosis Study were included. Medical and lifestyle history was obtained by interview. 1243 total incident fractures were reported between year one and ten via yearly questionnaire, and confirmed by X-ray ( $13 \%$ hip, $4 \%$ pelvic, $12 \%$ clinical vertebral, 37\% upper limb, $24 \%$ lower limb, 10\% rib). Most fractures occurred in women aged $\geq 50$ years with a BMD T-score $\leq-1.0$ SD. Repeated measures ANOVA identified decreased physical activity participation between baseline, year five, and year ten $(\mathrm{F}(2,5503)=61.54$, $\mathrm{p}<0.001)$. Decreased physical activity in people who reported an incident fracture compared with no fracture was only significant at year ten $(\mathrm{F}(2,5503)=7.24, \mathrm{p}<0.01)$. In adjusted multivariable regression analyses, having a hip or pelvic fracture between year one and five was significantly associated with decreased physical activity at five-year follow up in people aged $\geq 50$ years $(-1857.87$ MET-mins/week; 95\% CI $[-2587.46,-1128.29])$. Other fracture types or multiple fractures of any type occurring between years one to five, and years five to ten were not associated with physical activity at five or ten-year follow-up.
Previous activity, age, body mass index, education, and number of comorbidities were significant correlates. Overall, history of hip or pelvic fracture may reduce physical activity over time.

\section{Examining cardiovascular risk metrics in osteoarthritic and non-osteoarthritic populations using CLSA data}

Y. Mei, ${ }^{1}$ M. Kadem, ${ }^{2}$ D. Kobsar, ${ }^{1}$ and B.K. Al-Khazraji ${ }^{1}$

${ }^{1}$ Department of Kinesiology, Faculty of Science, McMaster University, Hamilton, ON L8S4L8, Canada. ${ }^{2}$ School of Biomedical Engineering, McMaster University, Hamilton, ON L8S4L8, Canada.

Osteoarthritis (OA) is characterized by the progressive degradation of the joint that results in pain and disability and is often comorbid with cardiovascular disease (CVD). Surrogate measures of cardiovascular health include the carotid intima-media thickness (cIMT) and composite cardiovascular risk scores such as the Framingham Risk Score (FRS) (10-year risk score estimate for CVD onset). Modifiable factors not considered in the FRS such as physical activity, diet, and stress are encompassed by the recently developed InterHeart risk score (IHS). This study employed both cIMT as a subclinical marker of CVD risk and the FRS and IHS values as predictors of CVD risk to compare the accuracy of predicting CVD outcome in adults with OA $(n=773)$ and healthy age- and sex-matched cohort without OA ( $n=1546$ ) based out of the longitudinal cohort tracked by the Canadian Longitudinal Study on Aging. As an index of model accuracy, AUC values from the logistic regression fits with CVD events at follow-up ( 3 years from baseline) as the outcome were compared. In an age- and sex-matched cohort, the incidence of CVD in the OA population $(14.2 \%)$ was significantly greater $(\mathrm{p}=0.04)$ than the control (11.3\%). Logistic regressions of cIMT (AUC 0.535, group OR 1.293), cIMT+FRS (AUC 0.595, group OR 1.238), and cIMT+IHS (AUC 0.560, group $O R$ 1.273) tested using known follow-up outcomes suggest that the combination of cIMT and CVD risk scores can yield greater predictive power of CVD outcomes at the 3-year follow-up in adults either with or without OA but with no cardiovascular disease history at baseline. Using cIMT in addition to CVD risk prediction scores can aid in early detection of CVD in those with OA, and inform preventative measures for cardiovascular disease onset in an at-risk population like OA which has historically been treated as a "joint-centric" condition. (Supported by MIRA.)

HIIT me with your best shot: The role of exercise intensity on cognitive function throughout the lifespan

S. Mekari, ${ }^{1,2,3}$ D.S. Kimmerly, ${ }^{4}$ and H.F. Neyedli ${ }^{4}$

${ }^{I}$ Department of Family Medicine, Université de Sherbrooke, Sherbrooke, QC J1H 5N4, Canada. ${ }^{2}$ Centre de formation médicale du Nouveau-Brunswick, Pavillon J.-Raymond Frenette, Moncton, NB E1A 7R1, Canada.

${ }^{3}$ School of Kinesiology, Acadia University, Wolfville, NS B4P 2R6, Canada.

${ }^{4}$ Division of Kinesiology, School of Health and Human Performance, Faculty of Health, Dalhousie University, Halifax, NS B3H 4R2, Canada.

Healthy aging is often associated with a decline in cognitive function. With age, it has been shown that high-intensity interval training (HIIT) program could provide greater neurocognitive health when compared to a moderate-intensity continuous training (MICT) program. We hypothesized that HIIT would improve executive function more than MICT. We also hypothesized that, in older adults, the neural protection offered by a HIIT program would be similar to the cognitive performances of healthy young adults. Forty-three older adults (age $68 \pm 7$ yrs) were randomly assigned to an isocaloric 6-weeks (3 days/week) of HIIT [ $2 \times 20$ min bouts alternating between 15 s intervals at $100 \%$ of peak power output (PPO) and passive recovery ( $0 \% \mathrm{PPO})$; $n=24$ ], or MICT (34min at $60 \%$ PPO; $n=19$ ). Twenty-five younger adults (age $32 \pm 8$ yrs) served as a control group. Executive function was assessed at baseline (all three groups) and post training (both older adults' groups) via a computerized Stroop Task. Prior to training, there was a main effect of 
age where older adults had a slower response time in the executive components of the Stroop task than younger adults $(1273 \pm 45$ versus $995 \pm 34 p<0.004)$. Following training, only the HIIT group exhibited an improvement in reaction time ( $1339 \pm 40$ to $1086 \pm 35 \mathrm{~ms} ; p<0.001)$. Furthermore, there was no difference between the post-training response times of the HIIT group and the younger adults $(1086 \pm 35$ versus $995 \pm$ $34 \mathrm{~ms} ; \boldsymbol{p}<0.198)$. Despite age-related differences at baseline, HIIT, but not MICT improved executive function of older adults to the same level as young healthy adults. Exercise programs should consider using HIIT protocols in an effort to combat cognitive decline in older adults.

\section{Regulation of mitochondrial function by the UPRmt transcriptional regulator ATF4}

\section{J.M. Memme and D.A. Hood}

Muscle Health Research Centre, School of Kinesiology and Health Science, York University, Toronto, ON M3J 1P3, Canada.

ATF4 is a transcription factor that both preserves, and promotes, mitochondrial function by enhancing the expression of important mitochondrial chaperones and proteases when muscle is subjected to a stress stimulus, like exercise. Moreover, ATF4 plays a central role in facilitating in organelle turnover through the mitophagy-lysosome system. Previous studies from our lab have highlighted ATF4 induction in animal and cell culture models of muscle contractile activity and differentiation. However, it remains to be determined whether ATF4 is necessary for mitochondrial adaptations in skeletal muscle. Therefore, our aim was to determine whether ATF4 is required for the maintenance of mitochondrial function and adaptation following an acute $3 \mathrm{~h}$ bout of contractile activity (ACA), or after repeated bouts (4 days; CCA) in C2C12 myotubes in which ATF4 is either overexpressed (OE) or knocked down (KD). We observed an attenuated induction of PGC-1 $\alpha$ mRNA following ACA in ATF4 KD myotubes, indicating diminished drive for mitochondrial biogenesis. The mRNA expression of ATF5, which acts as a mitochondrial-nuclear retrograde signal under organelle stress conditions, was induced both in response to ACA as well as with ATF4 OE, and was reduced in the absence of ATF4. Mitophagy flux, measured by mitochondrial-localized LC3-II, was upregulated in ATF4 OE and KD myotubes basally, and was augmented in both control and ATF4 OE cells following ACA, but not in the absence of ATF4. Mitochondrial function measured via oxygen consumption rate, indicated an $\sim 20 \%$ reduction in maximal respiration rate (MRR) in ATF4 KD myotubes, that was restored to control levels with CCA. Conversely, ATF4 OE myotubes displayed a MRR that was similar to control cells basally, however following CCA MRR was elevated 1.7-fold when ATF4 was enriched, as opposed to only 1.3-fold in controls. Together, these data indicate a novel pathway mediated by ATF4 for the regulation of mitochondrial function and remodelling following contractile activity in muscle.

\section{The Impact of Exercise Training on Muscle Sympathetic Nerve Activity: A Systematic Review and Meta-Analysis}

Sarah E. Meyer, ${ }^{1}$ Lauren Maier, ${ }^{1}$ Brittany Matenchuk, ${ }^{1}$ Allison Sivak, ${ }^{2}$ Craig D. Steinback, ${ }^{1}$ and Margie H. Davenport ${ }^{1}$

${ }^{\prime}$ Program for Pregnancy and Postpartum Health, Neurovascular Health Laboratory, Women and Children's Health Research Institute, Alberta Diabetes Institute, Faculty of Kinesiology, Sport, and Recreation, University of Alberta, Edmonton, Alberta, Canada.

${ }^{2}$ H.T. Coutts Education \& Physical Education Library, University of Alberta, Edmonton, Alberta, Canada.

The purpose of this systematic review and meta-analysis was to examine the effects of exercise training on muscle sympathetic nerve activity (MSNA) in humans. Online databases were searched up to June 11, 2021. Randomized controlled trials (RCTs) reporting on the relevant population (adults $\geq 18$ years of age) where MSNA was directly assessed using microneurography, intervention (objective measures of frequency, duration, volume or type of exercise), duration of intervention (intervention longer than 2 weeks), comparator (no exercise control group), and outcomes (MSNA [total activity, burst frequency, burst incidence, amplitude], heart rate, blood pressure [systolic blood pressure, diastolic blood pressure, or mean blood pressure], aerobic capacity [maximal or peak oxygen consumption]).18 RCTs ( $\mathrm{n}=532$ individuals) were included. 16 studies reporting on 470 individuals demonstrated that the exercise training increased peak oxygen consumption by $3.82 \mathrm{ml} / \mathrm{kg} / \mathrm{min}$ ( $95 \%$ CI [2.96 to 4.68$]$, $\mathrm{I}^{2}=0 \%$ ). Following the intervention, the exercise group had a reduction in burst frequency (17 studies, $\mathrm{n}=468$, mean difference $[\mathrm{MD}]-6.95$; $95 \%$ CI $[-9.31$ to -4.52 ], $\mathrm{I}^{2}=68 \%$ ) and burst incidence (11 studies, $\mathrm{n}=301$, mean difference [MD] $-10.61 ; 95 \%$ CI [ -16.49 to -4.73$], \mathrm{I}^{2}=84 \%$ ). Blood pressure and heart rate was not different between the exercise and control groups. These findings suggest that exercise training of a sufficient dose to improve aerobic capacity can reduce muscle sympathetic nerve activity, even in the absence of changes in heart rate or blood pressure. (This project is funded by NSERC and the Christenson Professorship in Active Healthy Living)

\section{Sulforaphane and Urolithin A exert time-dependent effects on mitochondrial protein signaling in C2C12 myotubes}

\section{N. Moradi and D.A. Hood}

Muscle Health Research Centre, School of Kinesiology and Health Science, York University, Toronto, ON M3J IP3, Canada

Many reports have demonstrated the physiological effects of exercise on skeletal muscle, but fewer have explored the effects of nutraceutical supplementation in enhancing the influence of exercise on muscle health. Sulforaphane (SFN) is a phytochemical that is a powerful inducer of the Nrf-2-ARE pathway, a mechanism that upregulates cellular defences against oxidative stress. The metabolite Urolithin A (UroA), on the other hand, has developed a reputable role in regulating mitochondrial turnover, primarily via mitophagy. The purpose of this study is to characterize the timedependent effects of each of these agents on protein markers that participate in the antioxidant and mitophagy pathways in $\mathrm{C} 2 \mathrm{C} 12$ myotubes. Differentiated muscle cells were treated with two concentrations of each nutraceutical for $4 \mathrm{~h}, 24 \mathrm{~h}$, or $48 \mathrm{~h}$. The upstream kinase phospho-AMPK was activated by 2-fold as early as 4 hrs of treatment with UroA, which subsided by 24 and $48 \mathrm{~h}$. The 1.3-fold increase in the downstream autophagy marker p62, however, was only observed by the 48-hr mark. Protein content also fluctuated in a time-dependent manner with SFN treatment, as 1.5-fold increases in the transcription factor, Nrf-2, were observed only at $4 \mathrm{~h}$, while 4-5-fold increases in the downstream antioxidant proteins NQO1 and HO-1, were observed and maintained only after $24 \mathrm{~h}$ and $48 \mathrm{~h}$ of treatment. SFN treatment also resulted in a dose-dependent 1.3-fold increase in COX IV, an indicator of mitochondrial content, at $48 \mathrm{~h}$. Our preliminary results suggest that these agents exert independent, beneficial effects on muscle cells which can have cytoprotective effects, improve mitochondrial content, and impact the drive for mitophagy. These data also set the stage for an examination of the effect of these nutraceuticals, in combination with contractile activity, on mitochondrial turnover and function, with implications for exercise capacity and muscle health. (Supported by MHRC, NSERC, and CIHR.)

Reliability and validity of dietary assessment tools in a Canadian athlete population

O. Morello, ${ }^{1}$ L. McPhee, ${ }^{2}$ M. Kucab, ${ }^{1}$ N. Bellissimo, ${ }^{1}$

and J.O. Totosy de Zepetnek ${ }^{2}$

${ }^{I}$ School of Nutrition, Ryerson University, Toronto, ON M5B 2K3, Canada.

${ }^{2}$ Faculty of Kinesiology and Health Studies, University of Regina, Regina, SK S4S OA2, Canada

The rising popularity of nutrition applications that allow athletes and their clinicians - to track dietary intake and patterns necessitates 
evaluating their inter-rater reliability and validity. Evaluating interrater reliability will provide insight into whether individuals can enter nutrient data into the same app, and evaluating validity will reveal if these apps, which often rely on American data (e.g., USDA), are valid in Canadian populations. We evaluated the inter-rater reliability and validity of two free nutrition apps, Cronometer (CRO) and MyFitnessPal (MFP), among Canadian athletes. Two raters (OM \& LM) independently input 43 three-day food records into CRO and MFP, and one rater (OM) input each food record into the reference ESHA using the 2015 Canadian Nutrient File (CNF) database. CRO showed good to excellent relative reliability $\left(\mathrm{ICC}_{2,1}=0.779-0.998\right)$ and good absolute reliability for all nutrients, except iron (shift in means $[\Delta][95 \% \mathrm{CI}]=-0.7 \mathrm{mg}[-1.3$ to -0.1$], \mathrm{SEM}=0.3 ; \mathrm{p}=0.018))$. MFP showed moderate to excellent relative reliability $\left(\mathrm{ICC}_{2,1}=0.512-0.952\right)$, and poor absolute reliability for total $\mathrm{kcal}(\Delta[95 \% \mathrm{CI}]=225.5[138.9-312.0], \mathrm{SEM}=42.9$; $\mathrm{p}<0.001)$ and carbohydrates $(\Delta[95 \% \mathrm{Cl}]=23.7 \mathrm{~g}[9.5-37.9]$, SEM=7.0; $\mathrm{p}<0.002)$. Regarding validity, a one-way ANOVA revealed no differences between CRO, MFP, and the CNF for total kcal, macronutrients, cholesterol, sodium, sugars, and fiber ( $\mathrm{p}>0.05)$. Independent $\mathrm{t}$-tests revealed no differences between CRO and CNF for select micronutrients that weren't reported in MFP (e.g., vitD, vitB12, iron). Bland-Altman plots showed CRO underestimated kcal with smaller bias compared to MFP that overestimated kcal with greater bias (95\% Limit of Agreement $(\mathrm{LOA})=-314-292$ vs. $-445-649$, respectively). CRO is a reliable and valid alternative to the $C N F$ for athletes and clinicians who want to track dietary intake and patterns. MFP reported on fewer nutrients and proved to be less reliable for measures of kcal and carbohydrates, and less valid as evidenced by greater Bland-Altman LOA when compared against the CNF.

\section{Association between Exercise Levels and Physical Function after a 6-week Virtual Exercise Program for Breast Cancer Survivors Receiving Endocrine Therapy Treatment}

S.A. Myers, ${ }^{1}$ S. Weller, ${ }^{1}$ N. LeVasseur, ${ }^{2}$ C. Van Patten, ${ }^{2}$
and K.L. Campbell ${ }^{1}$
${ }^{1}$ Department of Rehabilitation Sciences, The University of British Columbia, Vancouver, BC V6T 1Z2,
Canada.
${ }^{2}$ Medical and Nutrition Oncology, British Columbia Cancer Agency, Vancouver, BC V5Z 1G1, Canada.

Hormone-receptor positive breast cancer $(\mathrm{BC})$ is the most prevalent cancer in females globally. Adjuvant endocrine therapy is standard care, however, approximately $40 \%$ of BC survivors discontinue treatment due to negative musculoskeletal (MSK) side-effects. Given the benefits of endocrine therapy to reduce recurrence risk and improve survival rates, strategies that improve medication adherence and MSK side-effects, are needed. Exercise may be one strategy to reduce MSK side-effects and improve physical function (PF). The purpose of this study was to explore the preliminary efficacy of a virtual exercise program on medication adherence and PF. The purpose of this analysis was to explore the preliminary association between change in exercise levels and change in PF. The study was a single-arm pilot study. Recruitment included self-referral or oncologist referral. A strength and aerobic exercise program was delivered virtually twice weekly via Zoom over 6-weeks. Physical function was assessed with the 30 second sit-to-stand test, and usual self-reported exercise levels were assessed by a modified Godin Leisure-Time Exercise Questionnaire (GLTEQ). Change from baseline to 6-weeks was examined using paired t-tests. The association between change in exercise levels and change in PF was assessed using Spearman Rank Correlation tests. Twenty-three individuals completed the intervention. Results from baseline to 6-weeks demonstrated a significant improvement in $\mathrm{PF}$ (reps) $(\mathrm{p}<0.001$, mean $=3.1 \pm 2.5)$ and minutes per week of selfreported strenuous intensity exercise $(\mathrm{p}=0.007$, mean $=25.4 \pm 42.2)$. A moderate effect was shown between decreases in light intensity exercise and increases in strenuous intensity exercise ( $\mathrm{rs}=-0.453$, $\mathrm{p}=0.034$ ). Reductions in light intensity exercise were significantly associated with improvements in PF ( $\mathrm{rs}=-0.495, \mathrm{p}=0.019)$. In conclusion, a 6-week supervised, virtual, group-based aerobic and resistance exercise program was associated with increased strenuous-intensity exercise levels and improved PF in women taking long-term endocrine therapy.

Correlates of physical activity in children from families speaking non-official languages at home: a multi-site Canadian study

R. Nayakarathna, ${ }^{1}$ N. Patel, ${ }^{1}$ C. Currie, ${ }^{1}$ G. Faulkner, ${ }^{2}$ N. Riazi, ${ }^{2}$ M.S. Tremblay, ${ }^{3}$ F. Trudeau, ${ }^{4}$ and R. Larouche ${ }^{1}$

${ }^{I}$ Faculty of Health Sciences, University of Lethbridge, Lethbridge, AB TIK 3M4, Canada.

${ }^{2} S$ chool of Kinesiology, The University of British Columbia, Vancouver, BC V6T IN1, Canada.

${ }^{3}$ Healthy Active Living and Obesity Research Group, CHEO Research Institute, Ottawa, ON KIH 8L1, Canada.

${ }^{4}$ Département des Sciences de l'Activité Physique, Université du Québec à Trois-Rivières, Trois-Rivières QC G8Z 4M3, Canada.

Previous research suggests that Canadian children from families who speak a non-official language at home are less physically active. Using data collected in three regions of Canada, we investigated the correlates of physical activity (PA) among children from families speaking non-official languages at home. The sample included 1,699 children recruited in 37 elementary schools and was stratified by area-level socioeconomic status (SES) and type of urbanization (urban, suburban, rural). Among these children, 478 spoke a non-official language at home. Steps/day and moderate- to vigorous-intensity physical activity (MVPA) were measured using pedometers for 7 consecutive days. Data on potential correlates were collected via child and parent surveys and weather data from Environment Canada. Gender-stratified linear mixed models were used to examine correlates of PA. We examined age and area-level SES as potential moderators of the association between PA and outdoor time. The strongest correlate of higher PA among boys and girls was outdoor time. Younger children were generally more active. The strength of the association between outdoor time and PA decreased with age in boys and increased with age in girls. Having a long-standing illness/disability and lower area-level SES was associated with less PA among boys. The differences in PA between boys attending schools in low- versus high-SES areas decreased with outdoor time. Girls who had less independent mobility and whose parents reported driving to work accumulated more MVPA. Outdoor time was the most consistent correlate of PA. Our findings suggest future interventions in this population should aim to increase opportunities to be active outdoors and address socio-economic disparities.

\section{A novel AMPK activator stimulates autophagy signaling in dystrophic skeletal muscle}

S.Y. Ng, A. Manta, M. Rana, and V. Ljubicic

Department of Kinesiology, McMaster University, Hamilton, ON, Canada.

Duchenne muscular dystrophy (DMD) is a life-limiting neuromuscular disorder characterized by muscle wasting and dysregulated autophagy. Previous studies have demonstrated the therapeutic potential of pharmacological activators of AMP-activated protein kinase (AMPK) in pre-clinical models of DMD. However, these compounds have yet to translate efficacy to DMD patients. Identifying clinically transferable AMPK agonists may provide novel therapies for DMD. $\mathrm{O} 304$ is an orally bioavailable pan-AMPK activator that attenuates metabolic disease in rodents and humans. The purpose of our study was to evaluate the molecular response to 0304 in dystrophic muscle. 6-week-old dystrophic mice (D2.mdx; $\mathrm{n}=12$ ) were gavaged with a single dose of $0304(100 \mathrm{mg} / \mathrm{kg}$; Betagenon) or with the vehicle (Veh) solution. Wild-type (WT) animals were given the Veh and served as healthy controls. Tibialis anterior muscles were 
collected 3-, 12-, or 24-hours post-gavage and subsequently processed for analyses. O304 administration in dystrophic animals significantly elevated (+98\%) AMPK activation status 12-hours postgavage, which returned to baseline levels by 24-hours. Phosphorylation levels of the AMPK downstream network, including TBC1D1 ${ }^{\text {Ser700, }}$ p38 ${ }^{\text {Thr180/182 }}$, and FOXO3a ${ }^{\text {Ser588 }}$, were $\sim 2$-fold higher $(\mathrm{P}<0.05) 3$ hours following $\mathrm{O} 304$ administration as compared to Veh-treated animals. O304 also evoked a concomitant, significant increase $(+150-230 \%)$ in PGC-1 $\alpha$ mRNA. Collectively, these data demonstrate O304 effectively stimulated AMPK signaling in dystrophic muscle. Regarding autophagy, the activation status of AMPK-specific ULK ${ }^{\text {Ser555 }}$ was 2.4-fold greater $(\mathrm{P}<0.05)$ in $\mathrm{D} 2 . \mathrm{mdx}$ animals dosed with $\mathrm{O} 304$ compared with their Veh-treated littermates. Indicators of the mTORC1/ULK signaling axis, such as S6 and ULK ${ }^{\text {Ser757, were significantly elevated }(+180-210 \%)}$ in Veh-treated dystrophic animals versus their WT counterparts. O304 robustly reduced $(\mathrm{P}<0.05)$ dysregulated mTORC1/ULK signaling relative to the D2.mdx Veh group, to the point where O304-treated mice were similar to WT animals. These results suggest that 0304 elicited corrective autophagic signalling in dystrophic skeletal muscle. This ongoing work highlights AMPK-specific mechanisms evoked with a single dose of $\mathrm{O} 304$ and sets the stage for future pre-clinical investigation in DMD.

\section{The influence of social engagement during exercise on associated cognitive and affective changes among older adult women}

T.H. Nieman, ${ }^{1}$ M. Bergelt, ${ }^{2}$ and L.E. Middleton ${ }^{2}$

${ }^{1}$ School of Kinesiology, Faculty of Health Sciences, University of Western Ontario, London, ON N6A 3K7, Canada.

${ }^{2}$ Department of Kinesiology and Health Sciences, Faculty of Health, University of Waterloo, Waterloo, ONN2L 3G1, Canado.

Concurrent social engagement may influence cognitive and affective changes observed after exercise. The objective here was to compare changes in cognitive control and affect between social exercise (SE) and independent exercise (IE) versus a control condition. Thirty healthy older adult women (mean age=69.9 [2.91]) completed 3 sessions in randomized order: 1) SE: moderate-intensity walking with concurrent conversation with another participant; 2) IE: moderate-intensity walking alone; and 3) informative podcast control (CO). Cognitive control was assessed with Flanker task accuracy-corrected response time (inverse efficiency score, IES) pre/ post-intervention. Affect was assessed using the Physical Activity Affect Scale pre/post and at 3-hour intervals for 34h. To explore variability in cognitive response to exercise according to personality, baseline physical activity, and conversation, four participant groups were compared: 1) Consistent Responders; 2) Social Responders; 3) Solo Responders; 4) Non-Responders. There was no condition*time interaction for IES $(\mathrm{F}(2,317)=1.49, \mathrm{p}=.227)$. IES featured a main effect of condition with lower IES in SE compared to CO $(F(2,317)=3.23$, $\mathrm{p}=.037)$ but not compared to IE ( $\mathrm{p}=.774)$. There was a condition*time interaction for positive affect $(\mathrm{F}(2,143)=5.15, \mathrm{p}=.007)$ where positive affect improved pre- to post-intervention following SE $(\mathrm{p}=.009)$ but not IE $(\mathrm{p}=.991)$ or CO $(\mathrm{p}=1.0)$. Negative affect showed a condition* time interaction $(\mathrm{F}(8,823)=2.3, \mathrm{p}=.019)$ with a gradual reduction from pre-intervention until 6 h-post $(\mathrm{F}(8,823)=2.3, \mathrm{p}=.003)$ following $\mathrm{SE}$, but not IE $(\mathrm{p}=1.0)$ or $\mathrm{CO}(\mathrm{p}=.628)$. Exploratory analyses suggested that those responsive to SE were more extroverted $(F(3,356)=3.13$, $\mathrm{p}=.026)$, conscientious $(\mathrm{F}(3,356)=5.18, \mathrm{p}=.002)$, and agreeable $(\mathrm{F}(3,356)=$ 9.24, $\mathrm{p}<.001$ ) than other groups. These groups also perceived high quality conversation $(\mathrm{F}(3,356)=23.5, \mathrm{p}<.001)$ but contributed less in conversation $(\mathrm{F}(3,332)=10.2, \mathrm{p}<.001)$. This study suggests that social engagement during exercise is an effective strategy to promote affective gains in older women. Exploratory analysis suggests that individual and social conversation characteristics might moderate cognitive response to exercise.
Sympathetic inhibition during mild and moderate dynamic leg exercise in healthy middle-aged subjects: contributing mechanisms

\author{
C.F. Notarius, ${ }^{1,2,3}$ M.B. Badrov, ${ }^{1,2}$ D.A. Keir, ${ }^{1}$ E. Keys, ${ }^{1,2}$ \\ and J.S. Floras ${ }^{1,2,3}$ \\ ${ }^{\prime}$ Division of Cardiology, University Health Network, Toronto, ON M5G 2C4, Canada \\ ${ }^{2}$ Sinai Health, Toronto, ON M5G1X5, Canada. \\ ${ }^{3}$ University of Toronto, Toronto, ON, Canada.
}

We recently showed that ageing augments both resting muscle sympathetic nerve activity (MSNA), as well as sympathoinhibition during mild and moderate dynamic 1-leg exercise observed in young healthy subjects. In order to evaluate which reflexes contribute to this augmentation in older subjects, we recruited 11 (6 women) healthy medication-free volunteers (mean age $58 \pm 2 \mathrm{SE}$ [range 49 to 72] years); assessed their peak oxygen uptake by open circuit spirometry $\left(\mathrm{VO}_{2 \text { peak }}\right)$; and, on a separate day, measured heart rate (HR), blood pressure (BP) and fibular MSNA (microneurography) at rest and during upright 1-leg cycling ( 2 min each unloaded and at $30-40 \%$ of $\dot{\mathrm{VO}}_{2 \text { peak }}$ ) followed by $2 \mathrm{~min}$ of post-exercise circulatory occlusion (PECO) to isolate the effect of the muscle metaboreflex. We repeated the cycling protocol during supine exercise (to increase sympathoinhibitory cardiopulmonary baroreflex activation) and again during upright cycling while breathing hyperoxic air $\left(32 \% \mathrm{O}_{2}\right.$ : to suppress the sympathoexcitatory arterial chemoreceptor). Both supine posture and hyperoxia attenuated MSNA burst frequency at rest compared with upright posture alone (main effect $\mathrm{P}=0.02$ ). MSNA burst frequency progressively decreased in an intensity dependent manner while cycling upright $(\mathrm{P}=0.01)$, with no metaboreflex effect $(\mathrm{P}=0.31 \mathrm{PECO}$ vs loaded cycle). It was also reduced during mild but not moderate supine cycling $(\mathrm{P}<0.05$ vs pre exercise) but unchanged during hyperoxia. Similarly HR and systolic and diastolic BP were lowest during supine rest and mild exercise (interaction $\mathrm{P}=0.02$; 0.003; and 0.006 respectively) with diastolic $\mathrm{BP}$ significantly higher during hyperoxia at rest $(\mathrm{P}=0.02)$ but not exercise. In contrast to previous work in young subjects, both the cardiopulmonary baroreflex and the arterial chemoreflex contribute to the elevated resting MSNA of healthy middle-aged humans but only the former contributes to sympathoinihibition during mild dynamic leg exercise. (Funded by the Heart and Stroke Foundation of Canada and CIHR)

\section{The Effect of Sex on Neurovascular Recovery from Acute Stress}

H.R. Orr, ${ }^{1}{ }^{\text {Y. Coovadia, }}{ }^{1}$ T.E. Adler, ${ }^{1}$ and C.W. Usselman ${ }^{1,2}$

${ }^{I}$ Cardiovascular Health and Autonomic Regulation Laboratory, Department of Kinesiology and Physical Education, Faculty of Education, McGill University, Montreal, QC H2W 1S4, Canada.

${ }^{2}$ Mc Gill Research Centre for Physical Activity and Health, Mc Gill University, Montreal, QC, Canada.

Exaggerated sympathetic responses to acute stress are associated with elevated cardiovascular disease (CVD) risk. Interestingly, CVD is more prevalent in young men than premenopausal women, yet sex differences in muscle sympathetic nerve activity (MSNA) during acute stress are inconsistent. However, sex differences in MSNA during the recovery period immediately following acute stress have yet to be established. Therefore, we tested the hypothesis that MSNA would be greater in men than women during recovery from the cold pressor test (CPT; 3-min immersion of a limb in ice-water) and apnea (maximal voluntary breath-hold). We tested 10 men and 9 women $(23 \pm 3$ vs $22 \pm 2 \mathrm{yrs}$, respectively, $\mathrm{p}=0.42)$. The stressors were each followed by a 3-min recovery period, analyzed in 1-min bins. Men and women demonstrated similar MSNA (peroneal nerve microneurography, NeuroAmp, ADInstruments) during baseline (18 \pm 9 vs $14 \pm 7$ bursts/100 heartbeats, $\mathrm{p}=0.32)$, during CPT $(42 \pm 14$ vs $32 \pm 18, \mathrm{p}=0.19$ ), and during apnea ( $35 \pm 14$ vs $40 \pm 16, \mathrm{p}=0.44$ ). During recovery from CPT, MSNA decreased in both sexes (main effect of time: $\mathrm{p}<0.01$ ) but was higher across all time points in men than women (main effect of sex: $\mathrm{p}=0.02$ ). Conversely, no sex differences 
were observed during apnea recovery (time: $p<0.01$, sex: $p=0.29$, time by sex: $\mathrm{p}=0.28$ ). These findings suggest that while sympathetic recovery from acute chemoreflex activation is similar between the sexes, men exhibit greater MSNA during recovery from the CPT, an acute pain stimulus. MSNA remained elevated after the CPT longer in men than women, suggesting one mechanism which may contribute to the higher risk of CVD in men.

Criterion-validity of the Physical Activity Vital Sign for estimating habitual moderate-to-vigorous physical activity in active younger and older adults

\section{L.P. Pellerine, ${ }^{1}$ D.S. Kimmerly, ${ }^{1}$ J.R. Fowles, ${ }^{2}$ and M.W. O’Brien ${ }^{1}$ \\ ${ }^{I}$ Division of Kinesiology, School of Health and Human Performance, Faculty of Health, Dalhousie University, Halifax, NS, Canada. \\ ${ }^{2}$ Centre of Lifestyle Studies, School of Kinesiology, Acadia University, Wolfville, NS, Canada.}

The Canadian Society for Exercise Physiology (CSEP) recommends adults achieve $>\mathbf{1 5 0}$ minutes of moderate-to-vigorous physical activity (MVPA) per week to reduce their risk of chronic disease. Brief questionnaires can be used to quickly estimate MVPA levels in clinical and exercise counselling settings. The Physical Activity Vital Sign (PAVS) is a 2-question assessment developed to estimate weekly MVPA levels and is included in the CSEP Physical Activity Training for Health manual. However, it is unknown if the PAVS accurately estimates MVPA in active younger or older adults relative to a previously validated objective measure. The purpose of this study was to test the hypothesis that the PAVS is an accurate measure of MVPA levels among younger ( $n=174 ; 23 \pm 7$ years) and older ( $\mathrm{n}=56 ; 66 \pm 28$ years) adults. The waist-worn PiezoRx accelerometer served as the criterion measure and was worn for 7-days. Standardized physical activity monitor validation procedures were followed (correlations, Bland-Altman analyses, equivalence testing). In older adults, MVPA was similar $(\mathrm{P}=0.39)$ between the PAVS $(176 \pm$ $\left.127 \mathrm{mins} \cdot \mathrm{week}^{-1}\right)$ and PiezoRx $\left(201 \pm 108 \mathrm{mins} \cdot \mathrm{week}^{-1}\right)$ with neither a fixed (mean difference: $24 \pm 118$ mins $\cdot$ week $^{-1}$ ) nor proportional bias. The younger adult cohort presented contrasting results, as the PAVS underestimated $(\mathrm{P}<0.001)$ MVPA $\left(243 \pm 280\right.$ mins $\cdot$ week $\left.^{-1}\right)$ versus the PiezoRx $\left(365 \pm 159\right.$ mins.week $\left.{ }^{-1}\right)$ and demonstrated both fixed (mean difference: 135 mins.week ${ }^{-1}$ ) and proportional biases. The PAVS was a valid estimate of habitual MVPA in active older, but not active younger adults. Clinicians and exercise professionals are encouraged to use the PAVS with confidence in active older patients but are cautioned against its use in active younger adults. Further investigations into the criterion validity of the PAVS using a more representative population of less active adults are recommended.

Sympathetic neurohemodynamic transduction is attenuated in older males independent of age-related declines in aerobic fitness

J.L. Petterson, ${ }^{1}$ M.W. O’Brien, ${ }^{1}$ D.J. Ramsay, ${ }^{1}$ W. Johnston, ${ }^{1}$ C.D. O'Neill, ${ }^{2,4}$ S. Dogra, ${ }^{3,4}$ S. Mekary, ${ }^{4}$ J.S. Floras, ${ }^{5}$ and D.S. Kimmerly ${ }^{1}$

${ }^{I}$ Division of Kinesiology, School of Health and Human Performance, Faculty of Health, Dalhousie University, Halifax, NS, Canada.

${ }^{2}$ University of Ottawa Heart Institute, Ottawa, ON, Canada.

${ }^{3}$ Faculty of Health Sciences, University of Ontario Institute of Technology, Oshawa, ON, Canada.

${ }^{4}$ School of Kinesiology, Acadia University, Wolfville, NS, Canada

${ }^{5}$ University Health Network and Sinai Health System Division of Cardiology, Department of Medicine, University of Toronto and the Toronto General Research Institute, University Health Network, Toronto ON, Canada.

Muscle sympathetic nerve activity (MSNA) increases with age. Smaller vascular and pressor responses to MSNA (i.e., sympathetic neurohemodynamic transduction) have been observed in older versus younger males. Previous research has not considered the influence of aerobic fitness, which also attenuates sympathetic transduction. We tested the hypothesis that older males would have lower sympathetic neurohemodynamic transduction despite agerelated reductions in aerobic fitness. Relative peak oxygen consumption $\left(\mathrm{VO}_{2}\right.$ peak; via graded cycle ergometry) was assessed in 22 younger and 16 older males. Participants were subsequently matched based on age-normative reference percentiles resulting in a final sample of 14 younger ( $23 \pm 3 y r s, 47.0 \pm 10.1 \mathrm{ml} / \mathrm{kg} / \mathrm{min}, 61 \pm 34$ percentile) and 14 older males (64 $\pm 5 \mathrm{yrs}, 38.7 \pm 11.2 \mathrm{ml} / \mathrm{kg} / \mathrm{min}, 63 \pm 37$ percentile). Resting beat-by-beat mean arterial blood pressure (MAP; via finger photoplethysmography) and common fibular nerve MSNA recordings (via microneurography) were collected on a separate day. Absolute changes in MAP were tracked for 12 cardiac cycles following each burst of MSNA. The peak increase in MAP was used to quantify sympathetic transduction. Older males had blunted peak MAP $(0.9 \pm$ $0.9 \mathrm{mmHg})$ responses compared to younger males $(2.0 \pm 0.6 \mathrm{mmHg}$, $\mathrm{P}=0.001)$. In the entire unmatched cohort, there was an inverse relationship between peak MAP responses and relative $\mathrm{VO}_{2}$ peak for both younger $(\mathrm{R}=-0.430, \mathrm{P}=0.046)$ and older $(\mathrm{R}=-0.530, \mathrm{P}=0.035)$ males. These findings highlight the independent impact of aging on the attenuated pressor responses to resting sympathetic outflow in healthy males after controlling for normative aerobic fitness levels.

\section{Acute Effects of Milk vs Carbohydrate on Bone Turnover Biomarkers Following Exercise in Young Adult Females}

\author{
J.L. Prowting, ${ }^{1}$ L.E. Skelly, ${ }^{1}$ N. Kurgan, ${ }^{2}$ P. Klentrou, ${ }^{2}$ and A.R. Josse ${ }^{1,2}$ \\ ${ }^{I}$ School of Kinesiology and Health Science, York University, Toronto, ON, Canada. \\ ${ }^{2}$ Department of Kinesiology, Brock University, St. Catharines, ON, Canada.
}

Dairy products and impact exercise are known to be independently beneficial for bone mineral properties. It is unknown however whether combining these two osteogenic interventions can synergistically augment acute bone turnover and cellular responses. Using a randomized crossover design, our objective was to compare the acute effects of consuming milk vs an isoenergetic carbohydrate control beverage on bone biomarkers following loading exercise. Thirteen healthy female participants (Age $=20 \pm 2 \mathrm{y}$; Weight $=57 \pm 5 \mathrm{~kg}$ ) consumed either $555 \mathrm{~mL}$ of skimmed milk (MILK) or $52.5 \mathrm{~g}$ of maltodextrin within $500 \mathrm{~mL}$ of water (CHO), both immediately and 1 hour following completion of a combined plyometric and resistance exercise bout. Venous blood samples were obtained pre-exercise, and 15-min, 75-min, 24-h and 48-h post-exercise to assess serum concentrations of bone resorption biomarkers, including carboxylterminal crosslinking telopeptide of type I collagen (CTX), receptor activator nuclear factor kappa- $\beta$ ligand (RANKL), and sclerostin, as well as bone formation biomarkers, including osteoprotegerin (OPG) and osteocalcin. There was a significant main effect for time $(\mathrm{p}<0.05)$ for RANKL, OPG:RANKL ratio, osteocalcin and sclerostin, but no condition or interaction effects were observed $(\mathrm{p}>0.05)$. Post-hoc analysis revealed that at 75-min post-exercise, RANKL, osteocalcin and sclerostin were lower and OPG:RANKL ratio was higher compared to baseline in both conditions. There were no differences over time or between conditions for CTX or OPG $(\mathrm{p}>0.05)$. The net post-exercise area-under-the-curve (AUC) response relative to baseline (percentage change from 1-h to 48-h) was significantly lower in the MILK vs CHO condition for CTX (paired t-test, $\mathrm{p}=0.03$ ), however there were no significant differences between conditions for the other biomarkers ( $p>0.05$ ). These results suggest that milk does not alter absolute post-exercise concentrations of bone biomarkers compared to $\mathrm{CHO}$. However, the blunted relative exercise response of the bone resorption biomarker CTX in the MILK condition compared with $\mathrm{CHO}$ suggests a less catabolic acute response when milk is consumed following loading exercise. Further research is required to determine whether this observation would result in beneficial chronic bone outcomes. 
Fitness level- and sex-related differences in FMD responses

L. Rasica, ${ }^{1}$ E.C. Inglis, ${ }^{1}$ D. Iannetta, ${ }^{1}$ R.N. Soares, ${ }^{2}$ and J.M. Murias ${ }^{1}$

${ }^{I}$ Faculty of Kinesiology, University of Calgary, Calgary, AB T2N 1N4, Canada.

${ }^{2}$ Dalton Cardiovascular Research Center, University of Missouri, Columbia, MO 65203, Missouri, USA.

Reactive hyperemia (RH) is largely used for the investigation of conduit artery endothelial function using Doppler ultrasound (FlowMediated Dilation, FMD). However, traditional calculation of \%FMD (percent change from baseline to peak arterial diameter) could be affected by a potential mathematical bias induced by sex- and fitness level-related differences in the baseline arterial diameter and/or attenuated responsiveness as a result of structural adaptations to long term training. Thus, evaluating additional vascular/ hemodynamics responses to $\mathrm{RH}$ might be crucial for detecting fitness level- and sex-related differences. Fifteen chronically trained (CT: 8 MALES, 7 FEMALES) and sixteen untrained (UT: 8 MALES, 8 FEMALES) individuals participated in this study. Aerobic fitness $\left(\mathrm{V}_{2 \max }\right)$ was assessed during a cycling incremental exercise test to volitional exhaustion. FMD was performed on the lower limb investigating the superficial femoral artery. $\mathrm{VO}_{2 \max }\left(\mathrm{mL} \cdot \mathrm{kg}^{-1} \cdot \mathrm{min}^{-1}\right)$ was greater in CT vs UT $(\mathrm{p} \leq 0.0001)$ and in MALES vs FEMALES $(\mathrm{p}=0.001)$. Baseline artery diameter was greater in CT vs UT $(\mathrm{p} \leq 0.0001)$ but not in MALES vs FEMALES $(\mathrm{p}=0.088)$. Peak artery diameter was greater in CT vs UT $(\mathrm{p} \leq 0.0001)$ and MALES vs FEMALES ( $\mathrm{p}=0.031)$. \%FMD was not different between groups (CT MALES, 4.62 \pm 1.42 ; CT FEMALES, $4.15 \pm 2.23$; UT MALES, 5.10 \pm 2.53 ; CT FEMALES, 3.20 \pm 1.67$)$. Peak blood flow showed greater values in CT vs UT $(\mathrm{p} \leq 0.0001)$ and MALES vs FEMALES $(\mathrm{p}=0.032)$. RH blood flow AUC (area under curve) was greater in CT vs UT ( $\mathrm{p}=0.001)$. $\mathrm{VO}_{2 \max }$ was significantly correlated with peak blood flow ( $\mathrm{p} \leq 0.0001)$ and RH blood flow AUC $(\mathrm{p}=0.0004)$ but not with \%FMD ( $\mathrm{p}=0.241)$. \%FMD did not identify fitness- and sex-related differences in vascular responses. However, when alternative outcomes from the FMD analysis were considered, such as peak blood flow and RH blood flow AUC, fitness- and sex-related differences were detected. (J. M. Murias is supported by the Natural Sciences and Engineering Research Council of Canada and the Heart \& Stroke Foundation of Canada)

Effect of heat waves on 24-hour movement behaviour, thermal perception, and thirst in children and adults: a pilot study

N. Ravanelli, ${ }^{1}$ N. Morris, ${ }^{2}$ and S. Morrison ${ }^{3}$

${ }^{I}$ School of Kinesiology, Lakehead University, Thunder Bay, ON P7G 2N3, Canada.

${ }^{2}$ Human Physiology and Nutrition, University of Colorado Colorado Springs, Colorado Springs, CO 80918, USA.

${ }^{3}$ Faculty of Sport, University of Ljubljana, Ljubljana, Slovenia.

Children and adults differ in managing high ambient temperatures largely due to differences in their body proportions and heat loss mechanisms. When children play, they may be at greater health risk as body temperatures rise, especially if behavioural thermoregulation is compromised in small children who may not accurately assess their own thermal status. Thus, the present study aimed to evaluate 24-hour movement behaviour (i.e. physical activity, screen time and sleep), thermal perception, and thirst in children and adults on days when a local heat alert was broadcast. To date, 25 adults $(40 \pm 10 \mathrm{y} ; 13$ female) and 12 children ( $6 \pm 2 \mathrm{y} ; 6$ female) from Canada and Slovenia have completed online questionnaires during heat waves throughout June and July 2021. On heat alert days (maximum ambient temperature: $30.2 \pm 1.5^{\circ} \mathrm{C}\left[26.7^{\circ} \mathrm{C}-\right.$ $\left.33.54^{\circ} \mathrm{C}\right]$ ), children engaged in significantly more moderate (children: $107 \pm 90$ mins, adults: $56 \pm 53$ mins, $\mathrm{P}=0.03$ ) and vigorous (children: $62 \pm 46$ mins, adults: $19 \pm 33$ mins, $P=0.005$ ) physical activity compared to adults, and spent less time engaging in sedentary activities (children: $40 \pm 98$ mins, adults: $113 \pm 138$ mins, $\mathrm{P}=0.01$ ). Despite higher activity levels, thermal sensation $(\mathrm{P}=0.77)$ and thermal comfort $(\mathrm{P}=0.39)$ where not different between groups. Interestingly, children did report higher thirst sensation throughout the day compared to adults $(\mathrm{P}=0.03)$. These preliminary findings highlight that during periods of extreme heat, children report engaging in more physical activity and greater thirst, which may pose an increased risk for exertional heat illness compared to their adult counterparts.

\section{Screening Varsity Athletes for Sleep Quality and Sleep-Associated Behaviours: A Pilot Study}

L.J. Rebello, A.W. Roberts, A.M. Fenuta, M.E. Bodner, and A.T. Cote

School of Human Kinetics, Trinity Western University, Langley, BC V2Y 1Y1, Canada.

Sleep is important for physical and mental health and performance. The National Sleep Foundation recommending adults attain 7-9 hours of quality sleep. However, research indicates that university students and athletes often report sub-optimal sleep quality and quantity leading to adverse health outcomes, decreased performance, and/or increased injury risk. Varsity athletes, as a unique subset of the university student demographic, may be particularly affected by poor sleep hygiene. In addition to the psychological strains typically related to academic demands, athletes endure additional stresses from daily training, competition, and travel. Sleep quality and the personal behaviours contributing to it are understudied in varsity athletes. This study evaluated sleep quality, quantity, and sleep-specific behaviours in a cohort of varsity athletes using two athlete-tailored sleep questionnaires. Nineteen varsity soccer athletes (4 females) completed the 16-item Athlete Sleep Screening Questionnaire (ASSQ) and 18-item Athlete Sleep Behaviour Questionnaire (ASBQ) during the off-season. The ASSQ assigns a score based on an athlete's quantity, sleep quality, and insomnia while the ASBQ highlights sleep-specific habits thought to hinder sleep attainment. The ASSQ indicated that $47 \%$ of athletes had a mild clinical sleep problem, $11 \%$ a moderate clinical sleep problem, and $16 \%$ a severe clinical sleep problem. Chronotype was split with $42 \%$ of the athletes characterized as a "morning-person", and $58 \%$ as an "evening-person", which has relevance if the training schedule is in opposition to the athlete's natural preference. The ASBQ unveiled that the most common behaviours associated with poor sleep were consistent use of light-emitting devices before bed (84\%), worrying about events before sleep (63\%), and going to bed physically sore (84\%). Our findings suggest that sleep hygiene in this pilot sample of varsity soccer athletes was suboptimal. Further research should evaluate sleep behaviours in varsity athletes representing various sports, and through key phases of the year to better understand sleep quality and quantity attainment, and impact on recovery.

\section{Tafazzin deficiency attenuates maximal mitochondrial respiration in $\mathrm{C} 2 \mathrm{C} 12$ myotubes}

Brandon J. Richards, Ashley N. Oliveira, and David A. Hood

Muscle Health Research Centre, School of Kinesiology and Health Science, York University, Toronto, ON, Canada.

Cardiolipin (CL) is a phospholipid predominately found in the inner mitochondrial membrane that has several integral roles. Arguably its most important functions are to stabilize complex III and complex IV of the electron transport chain (ETC), as well as to bind and interact with cytochrome $\mathrm{c}$. The biosynthesis of CL is governed by a number of enzymes. After nascent CL is produced, it is remodeled into its mature form by Tafazzin (Taz). This enzyme is a transacylase that is responsible for transferring acyl chains to immature CL, which completes the maturation process. The absence of Taz causes a cardiomyopathy termed Barth Syndrome. However, its role in regulating oxygen consumption and mitochondrial function in skeletal muscle is not known. Therefore, the purpose of this study is to investigate how oxygen consumption is affected by Taz deficiency 
in skeletal muscle cells. We knocked down Taz protein levels using an siRNA technique in C2C12 myotubes and measured mitochondrial respiration using Seahorse technology. Taz protein levels were depleted to $23 \%$ of scramble-treated cells. In the absence of Taz, we found that maximal oxygen consumption and ATP production were significantly reduced by $18 \%$ and $14 \%(\mathrm{P}<0.05)$, respectively, while basal respiration was unaffected. These data suggest that the formation of mature CL is imperative for mitochondrial function under stressful conditions such as exercise but may be less important to support resting metabolism. To our knowledge, this is the first study to illustrate that mitochondrial function is compromised by Taz deficiency in skeletal muscle. Future research should investigate whether exercise has the ability to rescue mitochondrial function in the absence of Taz.

\section{Health-related criterion-referenced cut-points for} cardiorespiratory fitness among youth: A systematic review

\author{
S. Rollo, ${ }^{1}$ B.J. Fraser, ${ }^{2}$ N. Seguin, ${ }^{1}$ M. Sampson, ${ }^{1}$ J.J. Lang, ${ }^{3}$ \\ G.R. Tomkinson, ${ }^{4}$ and M.S. Tremblay ${ }^{1}$
}

${ }^{I}$ Healthy Active Living and Obesity Research Group, Children's Hospital of Eastern Ontario Research Institute, Ottawa, ON K1H 8L1, Canada.

${ }^{2}$ Menzies Institute for Medical Research, University of Tasmania, Hobart, TAS 7000, Australia.

${ }^{3}$ Centre for Surveillance and Applied Research, Public Health Agency of Canada, Ottawa, ON K1A OK9, Canada.

${ }^{4}$ Department of Education, Health and Behavior Studies, University of North Dakota, Grand Forks, ND 58202, USA

The purpose of this systematic review was to identify health-related criterion-referenced cut-points for cardiorespiratory fitness (CRF) among youth aged 5-17 years. A systematic search of two electronic databases (MEDLINE and SPORTDiscus) was conducted in September 2020. Only peer-reviewed studies that developed health-related criterion-referenced cut-points for CRF among youth were eligible provided they included: (a) at least one quantitative assessment of CRF (e.g., $\mathrm{VO}_{2 \text { peak }}$ ); (b) at least one quantitative assessment of health (e.g., cardiometabolic risk); (c) a criterion for health; and (d) a quantitative analysis (e.g., receiver operating characteristic [ROC] curve) of at least one health-related cut-point for CRF. A narrative synthesis was used to describe the results of included studies. Collectively, 29 included studies developed health-related criterion-referenced cut-points for CRF among 193311 youth from 23 countries. Overall, $\mathrm{CRF}$ cut-points, expressed as $\mathrm{VO}_{2 \text { peak }}$, demonstrated moderate-to-high discriminatory ability (median area under the curve [AUC]: $\geq 0.64$ ) for cardiometabolic risk, obesity risk, and high blood pressure. Criterion-referenced cut-points for healthy CRF fell between 37.6 and $51.1 \mathrm{~mL} \cdot \mathrm{kg}^{-1} \cdot \mathrm{min}^{-1}$ for boys and between 32.0 and $49.5 \mathrm{~mL} \cdot \mathrm{kg}^{-1} \cdot \mathrm{min}^{-1}$ for girls aged 6-17 years. Currently, there is too wide a range of healthrelated criterion-referenced cut-points for CRF among youth to suggest universal age- and sex-specific thresholds. To further inform the development of universal cut-points, there is a need for additional research, using standardized testing protocols and health-risk definitions, that examines health-related criterion-referenced cut-points for CRF that are age, sex, and culturally diverse. (Funded by the Public Health Agency of Canada \#4500414210.)

\section{Prevalence and correlates of meeting the Canadian 24-Hour Movement Guidelines among a sample of Canadian parents during the COVID-19 pandemic}

\section{S. Rollo, A. Sckrapnick, and M.S. Tremblay}

Healthy Active Living and Obesity Research Group, Children's Hospital of Eastern Ontario Research Institute, Ottawa, ON K1H 8LI, Canada.

This study examined the prevalence and correlates of meeting vs. not meeting the individual and integrated recommendations within the Canadian 24-Hour Movement Guidelines among a representative sample of Canadian parents during the second wave of the COVID-19 pandemic. Participants were 1,477 respondents (parents of children aged 5-17 years) from a cross-sectional survey conducted in October 2020 by ParticipACTION, a national non-profit Canadian organization that promotes physical activity among Canadians. A total of 23 self-reported socio-demographic and behavioural correlates, including parental demographics $(n=8)$, child demographics $(n=4)$, change in family movement behaviours/characteristics $(\mathrm{n}=8)$, and COVID-19 related factors $(n=3)$ were assessed. Participants were classified as meeting or not meeting each of the time-specific recommendations for moderate-to-vigorous physical activity (MVPA), recreational screen time, and sleep duration, based on self-reported measures. The proportion of parents who met the MVPA, recreational screen time, sleep duration and integrated guidelines were 21.2, 51.0, 66.1, and 8.1\%, respectively. Multiple logistic regression analyses showed that being a parent $\geq 45$ years, having a university education, and higher levels of outdoor play were associated with meeting the integrated guidelines. Examining adherence to the individual behaviour recommendations, age, dwelling type, family hobbies, and outdoor play were associated with meeting the MVPA recommendation; employment status, education level, dog ownership, children's age, family physical activity, and levels of distress were associated with meeting the recreational screen time recommendation; and geographical region, education level, dwelling type, and levels of distress were associated with meeting the sleep duration recommendation. Few Canadian parents are meeting the integrated 24-Hour Movement Guidelines recommendations for MVPA, recreational screen time, and sleep during the COVID-19 pandemic. Numerous socio-demographic, behavioural, and COVID-19 related factors emerged as significant correlates of meeting vs. not meeting the individual and/or integrated recommendations within the guidelines.

Muscle power mediates deficits in the tibia bone strength, area and mass in children with autism spectrum disorder

M. Rostami Haji Abadi, ${ }^{1}$ J.D. Johnston, ${ }^{2}$ and S. Kontulainen ${ }^{1}$

${ }^{a}$ College of Kinesiology, University of Saskatchewan, SK S7N 5B2, Canada.

${ }^{b}$ College of Engineering, University of Saskatchewan, SK S7N 5A9, Canada.

Preliminary evidence indicates that children with autism spectrum disorder (ASD) have lower bone and muscle strength than typically developing children (TDC). We hypothesized that muscle strength, power and area account for deficits in bone strength, area and mass in children with ASD. Study objectives were to compare bone and muscle properties between children with ASD and TDC; and to explore mediation effects of muscle strength, power and area on bone differences. We recruited 16 children with ASD (mean age 10.1, SD 2.7 yrs) and 168 TDC $(10.9,1.8)$. We measured bone strength, area and mass at the distal and shaft sites of radius and tibia, and forearm and lower leg muscle areas using pQCT. We measured grip strength (GS) and long jump (LJ) distance (estimate of leg muscle power). We used site-specific MANCOVA to test bone and muscle differences and explore mediating effects of muscle outcomes on bone differences between the groups (sex, weight, limb length and muscle area as covariates). Children with ASD had -9 to $-20 \%$ lower bone strength, total and cortical area, and cortical mass at the radius and tibia shafts, and -9 to $-15 \%$ lower bone strength, total area and mass at the distal tibia. Children with ASD had $-25 \%$ lower LJ distance. GS and muscle areas did not differ between the groups $(\mathrm{P}>0.05)$. LJ distance accounted for 36 to $60 \%$ of deficits in bone strength, cortical area and mass at the tibia shaft, as well as bone strength, total area and mass at the distal tibia. Lower muscle power may contribute to bone deficits in children with ASD. Exercise interventions with games and activities including muscle strengthening are warranted to improve bone health in children with ASD. 


\section{UW WELL-FIT Cancer Exercise Program: 20 Years and Thriving}

C.A. Russell, S. Cazzola, J. Fraser, H. Gibson, L.A. Kraemer, S. Haghverdian, M. Mourtzakis, S. Pitton, E. Smith, and J. Stevens

Center for Community, Clinical and Applied Research Excellence. Department of Kinesiology and Health Sciences, University of Waterloo, Waterloo ON N2L 3G6, Canada

The UW WELL-FIT program, originally launched in 2002, has seen over 1900 enrolled participants in its twice weekly, supervised in-person program. Prior to the pandemic, our team of Kinesiologists and Clinical Exercise Physiologists (of $>19$ years experience) planned remote home-based exercise assessments, programming and behaviour change strategies for cancer patients. The purpose of this program was to address barriers that included access to communitybased oncology rehabilitation programs to the broader Waterloo Region. The framework included the development of an evidencebased, partially supervised, home-based exercise program targeting individuals with cancer who do not have access or who are unable to attend established in-person community-based exercise programs. As a result of the COVID-19 pandemic, we accelerated the implementation of our home-based exercise programming. UW WELL-FIT@HOME, a program for breast cancer patients, and START-FIT@HOME, for all other types of cancer, were launched remotely in January 2021. Special considerations in developing these programs included evidence-based guidelines for cancer patients, comprehensive assessments and individualized exercise programs for each participant, home fitness equipment, on-going program support, behaviour change strategies to enhance compliance, and the development of social connections, all done remotely. To date, 40 participants have enrolled in UW WELL-FIT@HOME or START-FIT@HOME. Lessons learned will provide insight into effective remote program strategies for those undergoing treatment. Preliminary results suggested aggressive promotion of the program, implementing objective measures within the assessment, and developing a mentor program. The ability to offer remote high-quality, evidence-based exercise programming for those living with cancer offers the unique opportunity to reach those in many more communities. From traditional in-person to remote exercise programming, our goal is to refine our program model to meet the ongoing and future needs, looking ahead to the next 20 years of UW WELL-FIT. [Supported by Quebec Breast Cancer Foundation.]

\section{The effects of chemoradiation and resistance wheel running on performance and muscle stem/progenitor cell populations in a pediatric mouse model of rhabdomyosarcoma}

\section{O. Sanders, ${ }^{1}$ N. Collao, ${ }^{1}$ and M. De Lisio ${ }^{1,2}$}

'School of Human Kinetics, University of Ottawa, Ottawa, ON KIN 6 N5 Canada.

${ }^{2}$ Department of Cellular and Molecular Medicine, University of Ottawa, Ottawa, ON KIN 6N5 Canada.

Rhabdomyosarcoma (RMS) is a soft tissue malignancy of the skeletal muscle that occurs primarily in pediatric populations. Chemoradiation therapy for RMS can lead to impaired muscle growth and development which results in life-long alterations in body composition, skeletal muscle atrophy, and reduced physical performance. Chemoradiation depletes Fibro-adipogenic progenitors (FAPs) and Satellite cells (SCs) which prevents normal muscle development. The extent to which resistance exercise training can prevent these negative long-term consequences in cancer survivors remains unclear. To address this gap, RMS was injected into a single hindlimb of juvenile (3 wk.) C57B1/6 mice that underwent systemic chemotherapy followed by targeted, fractionated radiation therapy to the injected limb. Following therapy, mice were exposed to resistance exercise training (RET; $n=10$ ) or remained sedentary (SED; $\mathrm{n}=10$ ) for 8 weeks. Body composition and performance tests were completed at pre and post therapy and exercise intervention. To directly evaluate the effects of RMS+therapy on SC fate and FAP paracrine function, cells were cultured alone (RMS) or with FAPs (RMS+FAPs) and exposed to radiation (IR) or sham conditions (CON). Conditioned media from these four conditions was applied to myoblasts for functional analyses. RET mice had lower body mass and body fat percentage and higher percentage of lean mass post-training compared to SED (all $\mathrm{p}<0.05$ ). RET mice had higher endurance post-training compared to SED ( $<<0.05)$. Conditioned media from RMS+FAPs resulted in more myoblast proliferation compared to RMS alone regardless of radiation exposure $(\mathrm{p}<0.05)$. Myoblast fusion index was lower when supplemented with RMS+ IR conditioned media than RMS media $(\mathrm{p}<0.05)$. FAPs rescued this radiation-induced defect. Thus, resistance exercise improves physical performance and body composition in vivo, while factors secreted from FAPs improve myogenesis in vitro. These data provide rationale for future investigations into the specific mechanisms responsible for these effects. (Funding provided by NSERC.)

\section{Airway size and its association with exertional dyspnoea in healthy males and females}

M.A.T. Sawatzky, ${ }^{1}$ Y. Molgat-Seon, ${ }^{1,2}$ P.B. Dominelli, ${ }^{3}$ M. Kirby, J.A. Guenette, ${ }^{3,5,6}$ J. Bourbeau, ${ }^{7}$ W.C. Tan, ${ }^{8}$ and A.W. Sheel ${ }^{6}$; on behalf of the CanCOLD collaborative research group

${ }^{I}$ Department of Kinesiology and Applied Health, University of Winnipeg, Winnipeg, MB R3B 2E9, Canada. ${ }^{2}$ Centre for Heart and Lung Innovation, St. Paul's Hospital, Vancouver, BC V6Z 1Y6, Canada. ${ }^{3}$ Department of Kinesiology, University of Waterloo, Waterloo, ON N2L 3G1, Canada. ${ }^{4}$ Department of Physics, Ryerson University, Toronto, ON M5B 2K3, Canada.

${ }^{5}$ Department of Physical Therapy, The University of British Columbia, Vancouver, BC V6T 1Z3, Canada. ${ }^{6}$ School of Kinesiology, The University of British Columbia, Vancouver, BC V6T IZ1, Canada. ${ }^{7}$ Department of Medicine, McGill University, Montreal, QC H4A 3J1, Canada.

${ }^{8}$ Department of Medicine, The University of British Columbia, Vancouver, BC V5Z 1 M9, Canada

In healthy adults, the large conducting airways are smaller and the perception of dyspnoea at absolute exercise intensities is higher in females than in males. We sought to determine whether sex-differences in airway calibre are related to sex-differences in exertional dyspnoea. We analyzed data from $n=90$ healthy $\geq 40$ y old never-smokers enrolled in the CanCOLD study who underwent pulmonary function testing, a chest computed tomography scan, and an incremental cycle exercise test. Males and females were matched into $n=26$ pairs based on age and total lung capacity (TLC). Despite being matched for TLC, the trachea, right main bronchus, left main bronchus, bronchus intermedius, left upper lobe, and left lower lobe had smaller luminal areas $(21-43 \%$; all $\mathrm{p}<0.01)$ and the airway size-to-lung size (dysanapsis) ratio was lower $($ males $=0.0330 \pm 0.0035$, females $=0.0315 \pm 0.0030$; $\mathrm{p}<0.05)$ in females compared to males. There were no sex-differences in dyspnoea (Borg CR10 scale) at the highest equivalent submaximal exercise work rate $(60 \mathrm{~W}$; males $=1.2 \pm 1.0$, females $=1.3 \pm 1.1 ; \mathrm{p}=0.48)$ or at peak exercise (males $=5.6 \pm 2.4$, females $=4.9 \pm 2.4 ; \mathrm{p}=0.26$ ), and dyspnoeaminute ventilation (dyspnoea- $\dot{V}_{\mathrm{E}} ; \mathrm{p}=0.31$ ) and dyspnoea-oxygen uptake (dyspnea-V $\mathrm{O}_{2} ; \mathrm{p}=0.15$ ) slopes were similar between the sexes. There was no significant association between dysnapsis ratio and dyspnoea- $\dot{V}_{\mathrm{E}}$ $(\mathrm{r}=0.05 ; \mathrm{p}=0.08)$ or dyspnoea- $\mathrm{V}_{2}(\mathrm{r}=0.09 ; \mathrm{p}=0.09)$. Our findings suggest that sex-differences in airway size do not contribute to sex-differences in exertional dyspnoea.

Muscle quality and physical function in colon cancer patients who are complete adjuvant treatment: A pilot study

H.K. Schulte, ${ }^{1}$ L. Meyers, ${ }^{1}$ H.J. Lim, ${ }^{2}$ C.J. Mitchell, ${ }^{3}$ and K.L. Campbell ${ }^{4}$

${ }^{I}$ Rehabilitation Sciences Graduate Program, The University of British Columbia, Vancouver BC V6TIZ4, Canada.

${ }^{2}$ BC Cancer, Vancouver, BC V5Z4E6, Canada.

${ }^{3}$ School of Kinesiology, The University of British Columbia, Vancouver, BC V6T1Z4, Canada.

${ }^{4}$ Department of Physical Therapy, The University of British Columbia, Vancouver, BC V6T1Z4, Canada.

Sarcopenia was originally defined as the age-related loss of skeletal muscle mass. However, more recent consensus statements emphasise the importance of muscle strength and function. Muscle strength and function are not dependent solely on muscle mass, spurring further 
research into muscle composition and quality (e.g., skeletal muscle density assesses for adipose tissue infiltration). We conducted a pilot study to calculate sample sizes for a larger trial investigating the associations between skeletal muscle (mass and quality) and physical function in colon cancer patients, using Pearson correlation coefficients. Computerized tomography scans were obtained when patients enrolled in the Canadian Cancer Trials Group CO.21 Trial, 2-6 months after adjuvant treatment. We obtained an image of the third lumbar vertebra to determine skeletal muscle area $\left(\mathrm{cm}^{2}\right)$ and density (Hounsfield Units (HU)), via SliceOMatic V5.0 (Tomovision, Montreal, Canada) manual tissue segmentation. Skeletal muscle index (SMI) was skeletal muscle area normalized for height $\left(\mathrm{cm}^{2} / \mathrm{m}^{2}\right)$. Skeletal muscle gauge (SMG) was SMI multiplied by density $\left(\mathrm{cm}^{2} \mathrm{xHU} / \mathrm{m}^{2}\right)$, integrating both mass and quality. Physical function measures were assessed during CO.21 Trial enrollment, including: 1) 30-second sit to stand (STT); 2) 30-seconds of arm curls; 3) 8-foot timed up and go (TUG); 4) 6-minute walk test (6MWT). In a subset of patients $(\mathrm{n}=16)$, SMI had higher associations than density or SMG with STT, arm curls and 6MWT. A moderate association between SMI and STT ( $r=.418, p=.107)$ generated a sample size of 42 for a larger trial (80\% power and $5 \%$ significance level). TUG displayed moderate associations with SMG and density; a sample size of 71 was generated to further investigate the relationship between SMG and TUG. Muscle quality provides increased information surrounding body composition, compared to mass alone. Research in larger cohorts is needed to determine the relationship between muscle quality and physical function in colon cancer patients.

Evaluating the associations between osteoporosis health beliefs and bone-specific physical activity and nutrition behaviors in young adults

A. Sevinc, J. Levee, X. Qi, N. Fripp, R. Andersen, and J.C. Gibbs

Department of Kinesiology and Physical Education, McGill University, Montreal, QC H2W 1S4, Canada.

Engaging in bone health promotion strategies including highimpact physical activity (PA) and adequate calcium intake in young adulthood are crucial for osteoporosis prevention. The Osteoporosis Health Beliefs Model is a theoretical framework that predicts health behavior with regards to a person's beliefs. We need to better understand which health beliefs are associated with bonespecific PA and nutrition behaviours in young men and women to inform gender-tailored bone health educational initiatives. The purpose of this cross-sectional study was to investigate the associations between osteoporosis health beliefs and bone-specific PA and nutrition behaviors in young adults aged $18-35$ years. Osteoporosis health beliefs, knowledge, and self-efficacy were assessed through the Osteoporosis Health Beliefs Scale, Osteoporosis Self-efficacy Scale and an osteoporosis knowledge scale, respectively. Bonespecific and moderate-to-vigorous PA (MVPA), and calcium intake were self-reported using the Bone-specific Physical Activity Questionnaire (BPAQ), International Physical Activity Questionnaire, and a calcium food frequency measure. Independent t-tests were used to compare outcomes by gender. Spearman correlation analyses were performed. Twenty-seven individuals participated in the study (63\% women, mean \pm SD age $22.09 \pm 2.321$ years, BMI $23.02 \pm 2.62 \mathrm{~kg} / \mathrm{m}^{2}$ ). Men reported greater MVPA than women ( $579 \pm 564$ vs. $264 \pm 179$ minutes/week, $p=0.041$ ), but no significant differences were found for BPAQ scores and calcium intake $(\mathrm{p}>0.05)$. There were no gender differences in health beliefs except men had greater calcium self-efficacy score than women (83.61 \pm 15.63 vs. $66.55 \pm 21.32, \mathrm{p}=0.037$ ). Osteoporosis knowledge and exercise self-efficacy were significantly correlated with past and total BPAQ scores $\left(\mathrm{r}_{\mathrm{s}}=0.400-0.448, \mathrm{p}<0.05\right)$; while health motivation and exercise self-efficacy scores were significantly correlated with MVPA $\left(r_{s}=0.492\right.$ and $\left.r_{s}=0.482, p<0.05\right)$. Calcium benefits score was associated with calcium intake $\left(\mathrm{r}_{\mathrm{s}}=0.593, \mathrm{p}=0.001\right)$. In conclusion, health motivation, calcium benefits, and exercise self-efficacy may be related to the translation of beliefs into bone-specific physical activity and nutrition behaviours in young adulthood. Multimodal educational programs could target health beliefs to increase engagement in osteoporosis prevention in young men and women.

Physical activity and sedentary time in children with concussion in comparison to age-, sex-, and season-matched healthy controls

Bhanu Sharma, ${ }^{1}$ Joyce Obeid, ${ }^{1}$ Carol DeMatteo, ${ }^{2,3}$ Michael D. Noseworthy, ${ }^{4,5,6,7}$ and Brian W. Timmons ${ }^{1,3}$

${ }^{I}$ Child Health and Exercise Medicine Program, Department of Pediatrics, McMaster University ${ }^{2}$ School of Rehabilitation Science, McMaster University.

${ }^{3}$ CanChild Centre for Childhood Disability Research, McMaster University. ${ }^{4}$ Imaging Research Centre, St. Joseph's Healthcare, Hamilton, ON, Canada. ${ }^{5}$ Department of Electrical \& Computer Engineering, McMaster University.

${ }^{6}$ McMaster School of Biomedical Engineering, McMaster University.

${ }^{7}$ Department of Radiology, McMaster University.

In recent years, the status quo of concussion management has changed. The former "rest-is-best" approach is being supplanted by an "exerciseis-medicine" mindset. However, our understanding of habitual physical activity in pediatric concussion remains limited. We do not know if there is a physical activity deficit in concussion and, if so, whether this inactivity contributes to concussion symptoms. This study was performed to characterize and quantify accelerometer-measured habitual physical activity and sedentary time in children with concussion in comparison to age-, sex-, and season-matched controls. We performed a secondary data analysis of 60 children with concussion (aged $12.74 \pm$ 2.85 years including 31 females, all within the first month of injury) and 1:1 matched healthy historical controls (aged $12.43 \pm 2.71$ years). We computed daily and hourly analyses of sedentary time, light physical activity (LPA), moderate physical activity (MPA), and vigorous physical activity (VPA). Hourly and daily activity and sedentary time were compared between groups and by sex using independent samples t-tests. Daily analyses showed that children with concussion were more sedentary than controls, with a mean difference (MD) of 38.3 minutes/day $(\mathrm{p}=0.006)$. Accordingly, they were also less active with respect to LPA $(\mathrm{MD}=-19.5$ minutes/day; $\mathrm{p}=0.008)$, MPA $(\mathrm{MD}=$ -9.8 minutes/day, $\mathrm{p}<0.001$ ), and VPA ( $\mathrm{MD}=-12.0$ minutes/day, $\mathrm{p}<0.001)$. Further, sex-specific analyses showed that girls with concussion were less active and more sedentary than both boys with concussion (MD, 50.8 minutes/day; $\mathrm{p}=0.010$ ) and healthy girls (MD, 51.1 minutes/day; $\mathrm{p}<0.010$ ). Hourly analyses showed that children with concussion were more sedentary and less active than healthy controls from 8:00 AM to 10:00 PM. This study helps quantify the physical activity deficit in pediatric concussion. It provides additional support for the continued study of physical activity interventions and/or guidelines aimed at reducing inactivity.

\section{Wearing of a facemask in ice-hockey playing youth during the COVID-19 pandemic does not affect performance}

\section{K.A. Shaw, ${ }^{1}$ J. Ko, ${ }^{1}$ A. Absher, ${ }^{1}$ G.A. Zello, ${ }^{2}$ S. Butcher, ${ }^{3}$ C. Tkachuk, ${ }^{4}$ and P.D. Chilibeck ${ }^{1}$}

${ }^{I}$ College of Kinesiology, University of Saskatchewan, Saskatoon, SK S7N 5B2, Canada

${ }^{2}$ College of Pharmacy and Nutrition, University of Saskatchewan, Saskatoon, SK S7N 5E5, Canada.

${ }^{3}$ School of Rehabilitation Science, University of Saskatchewan, Saskatoon, SK S7N 2Z4, Canada.

${ }^{4}$ Jim Pattinson Children's Hospital, Saskatoon, SK S7N 0W8, Canada.

During a game of ice hockey, players are regularly in close contact with each other, increasing the risk of spreading infectious diseases that travel through respiratory droplets, such as the SARS-CoV-2 virus. The close contact and increased breathing rates that occur during intense exercise have led to a high number of COVID-19 outbreaks within hockey teams across North America. Face masks are an effective measure in reducing the spread of respiratory droplets which, in turn, decreases the spread of such infectious diseases. Our previous research found that wearing a face mask during exercise 
has no impact on performance or oxygenation during short-duration, high-intensity exercise (Shaw et al. 2020; Int J Environ Res Public Health), but no known research has been carried out investigating the impact of wearing face masks on sports performance in children and youth, many of whom remain unvaccinated. The purpose of our research was to examine the effect of wearing a face mask during a progressive skating test in youth hockey players (9-14y). Twenty-four youth (19 males, 5 females, age $11.9 \pm 1.6 y)$ completed a progressive skating test (Yo-Yo IR1-IHmax) with and without a surgical mask in a randomized cross-over trial, while wearing full equipment. No differences were observed between the two conditions (mask $=290 \pm$ $119 \mathrm{~m}$ covered; sham mask $=301 \pm 104 \mathrm{~m}$ covered; $p=.85$ ). Our early results suggest that youth hockey players can wear a face mask while skating with no impact on their performance. Funded by the Jim Pattison Children's Hospital Foundation through the Saskatchewan Health Research Foundation

\section{A pea-powder supplement provided to female endurance athletes has potential to improve iron status}

\section{K.A. Shaw, ${ }^{1}$ G.A. Zello, ${ }^{2}$ D. Lindsay, ${ }^{3}$ T.D. Warkentin, ${ }^{3}$ J. Ko, ${ }^{1}$} and P.D. Chilibeck ${ }^{1}$

${ }^{I}$ College of Kinesiology, University of Saskatchewan, Saskatoon, SK S7N 5B2, Canada. ${ }^{2}$ College of Pharmacy and Nutrition, University of Saskatchewan, Saskatoon, SK S7N 5E5, Canada. ${ }^{3}$ Crop Development Centre/Department of Plant Sciences, University of Saskatchewan, Saskatoon, SK S7N 5A8, Canada.

Iron deficiency is the most prevalent nutrient deficiency worldwide, with $12 \%-16 \%$ of premenopausal women and $2 \%$ of men being affected. Females are more susceptible due to blood loss through menstruation and inadequate dietary iron intake. The risk for iron deficiency is greater in active individuals ( $30 \%$ of females and $11 \%$ of men) due to inflammation from training impacting absorption of iron, iron losses through sweating, or foot strike hemolysis (i.e., blood cells are damaged from repeated foot-ground contact during activities). Field peas are a rich source of iron, however, the naturally occurring plant molecule phytate chelates with iron, limiting iron absorption. Our purpose was to determine the feasibility of supplementing female runners with a field pea powder (49\% protein) derived from peas bred to have low phytate levels (i.e. higher iron bioavailability) and to assess the impact on iron status and exercise performance. Nine recreational female runners (32 $\pm 13 \mathrm{y} ; 63.3 \pm 13.8 \mathrm{~kg}$; $\mathrm{VO}_{2}$ max: $46.2 \pm 5.4 \mathrm{ml} / \mathrm{kg} / \mathrm{min}$ ) with low dietary iron intake $(<18 \mathrm{mg} /$ day $)$ were assessed for body composition, maximal aerobic power, $5 \mathrm{~km}$ time trial performance, and hemoglobin concentration before and after an 8-week dietary intervention, during which they were randomized to consume a powder made from regular peas, low phytate peas, or a placebo (maltodextrin). No differences were observed between groups for exercise performance or body composition; however, a group $\mathrm{x}$ time interaction was present for hemoglobin $(p=.03)$. Consuming maltodextrin decreased hemoglobin $(-5 \mathrm{~g} / \mathrm{L})$, whereas those consuming the regular phytate pea and low-phytate peas had a moderate increase $(+2 \mathrm{~g} / \mathrm{L})$, and large increase $(+11 \mathrm{~g} / \mathrm{L})$, respectively. Low-phytate pea powder was well tolerated, and our pilot results provide early evidence for improving hemoglobin concentration in female runners. Funded by Saskatchewan Ministry of Agriculture, Agriculture Development Fund and Saskatchewan Pulse Growers.

\section{Exercise-induced arterial hypoxemia in female masters athletes}

V.J. Shiffman, ${ }^{1}$ M.G. Leahy, ${ }^{1}$ S. Kipp, ${ }^{1}$ P. Rose,${ }^{2}$ B. Hughes, ${ }^{2}$ M.S. Koehle, ${ }^{1}$ J. McKinney, ${ }^{3}$ D.C. McKenzie, ${ }^{1}$ and A.W. Sheel ${ }^{1}$

${ }^{I}$ School of Kinesiology, The University of British Columbia, Vancouver, BC, Canada Department of Anesthesia, Vancouver Coastal Health, Vancouver, BC, Canada.

${ }^{3}$ Department of Cardiology, The University of British Columbia, Vancouver, BC, Canada

The pulmonary system can maintain arterial blood gas homeostasis during exercise in healthy, young adults. However, some endurance athletes demonstrate a significant reduction in arterial oxygenation during exercise, a phenomenon termed exercise-induced arterial hypoxemia (EIAH). EIAH has been previously observed in young adults, and male masters athletes but there are no reports of gas exchange impairment in female master athletes (FMA). We hypothesized the majority of FMA will develop EIAH during submaximal and maximal treadmill exercise. Pulmonary function was assessed followed by an incremental exercise test to determine maximal $\mathrm{O}_{2}$ uptake $\left(\mathrm{VO}_{2} \mathrm{max}\right)$. Participants were instrumented with a radial arterial catheter, an oesophageal balloon-tipped catheter and temperature probe. Arterial samples were drawn while participants exercised at $60-70,75,90-95$, and $100 \% \mathrm{~V}_{2}$ max for $2-4$ minutes. Participants ( $\mathrm{n}=6,48-57$ yo) had an average $\mathrm{VO}_{2}$ max of $47 \pm 2 \mathrm{~mL} / \mathrm{kg} / \mathrm{min}$ (range $40-55 \mathrm{~mL} / \mathrm{kg} / \mathrm{min}, 135-186 \%$ predicted). During submaximal and maximal exercise the arterial partial pressure of $\mathrm{O}_{2}\left(\mathrm{PaO}_{2}\right)$ decreased from rest by $19 \pm 2 \mathrm{mmHg}$ (range 14-24) and $16 \pm 4 \mathrm{~mm} \mathrm{Hg}$ (range 2021), respectively, with minimal change in oxyhemoglobin saturation $2.5 \pm 0.3 \%$ (range 1.9-3.4) and arterial $\mathrm{O}_{2}$ content $1.2 \pm 0.2 \mathrm{~mL} \mathrm{O}_{2} / 100 \mathrm{~mL}$ of blood (range 0.7-1.8) at all intensities. The arterial partial pressure of $\mathrm{CO}_{2}\left(\mathrm{PaCO}_{2}\right)$ decreased from rest by $4 \pm 1 \mathrm{mmHg}$ (range 2-6) and $5 \pm 1$ (range 3.5-6.0) at submaximal and maximal exercise, respectively. Those who had a minimal change to $\mathrm{PaCO}_{2}$ tended to have a greater reduction in $\mathrm{PaO}_{2}$. The alveolar to arterial oxygen $\left(\mathrm{A}-\mathrm{aDO}_{2}\right)$ gradient increased during submaximal (range $13-41 \mathrm{mmHg}$ ) and maximal (21$42 \mathrm{mmHg}$ ) exercise. There was a greater increase of $\mathrm{A}-\mathrm{aDO}_{2}$ related to a larger decrease in a $\mathrm{PaO}_{2}$. The decrease in $\mathrm{PaO}_{2}$ and increased $\mathrm{A}-\mathrm{aDO}_{2}$ gradient indicates an inadequate ventilatory response to exercise. These preliminary results suggest that FMA athletes develop EIAH at submaximal and maximal exercise intensities. (Supported by NSERC.)

\section{Impact of habitual sedentary patterns on popliteal artery endothelial-dependent vasodilation in healthy adults}

M.E. Shivgulam, M.W. O'Brien, J.A. Johns, J.L. Petterson, Y. Wu, R.J. Frayne, and D.S. Kimmerly

Division of Kinesiology, School of Health and Human Performance, Faculty of Health, Dalhousie University, Halifax, Nova Scotia, Canada.

Acute, laboratory-based bouts of prolonged sitting attenuate lowerlimb arterial endothelial-dependent vasodilation. However, the impact of habitual sedentary patterns on popliteal artery endothelial health is unclear. We tested the hypothesis that greater habitual total sedentary time, more time spent in prolonged sedentary bouts, and fewer sedentary breaks would be associated with worse popliteal flow-mediated dilation (FMD) responses. Ninety-eight healthy participants (19-77years, 53아) wore an activPAL monitor on the thigh for 6.4 \pm 0.8 -d to objectively measure sedentary activity and completed a popliteal ultrasound assessment to determine FMD. Both relative (\%baseline diameter) and absolute ( $\mathrm{mm}$ ) FMD were calculated. Using bivariate correlation and multiple regression analyses, we examined if there were relationships between sedentary outcomes and FMD while statistically controlling for any potential confounders. In the multiple regression model, age $(\mathrm{p}=0.006, \beta=-0.030,95 \% \mathrm{CI}=-0.051$, $-0.009)$ and total time in sedentary bouts $>1$-hour $(\mathrm{p}=0.031$, $\beta=-0.005,95 \% \mathrm{CI}=-0.009,-0.001)$ were independent predictors of relative FMD. Age $(\beta=-0.002,95 \% \mathrm{CI}=-0.003,-0.001)$, mean blood flow $(\beta=0.013,95 \% \mathrm{CI}=0.002,0.024)$, moderate-intensity physical activity $\left(\beta=155.9 \mathrm{E}^{-5}, 95 \% \mathrm{CI}=22.38 \mathrm{E}^{-5}, 289.4 \mathrm{E}^{-5}\right)$, sedentary breaks $(\beta=0.036$, $95 \% \mathrm{CI}=0.007,0.066$ ), and total time spent in sedentary bouts $>1$-hour $\left(\beta=-25.02 \mathrm{E}^{-5}, 95 \% \mathrm{CI}=-47.67 \mathrm{E}^{-5},-2.378 \mathrm{E}^{-5}\right)$ were predictors of absolute FMD (all, $\mathrm{p}<0.047$ ). All independent outcomes remained significant after partially controlling for all other predictor variables (all, $\mathrm{p}<0.031$ ). Habitual prolonged sedentary bouts and sedentary breaks, but not total sedentary time, were predictors of popliteal endothelialdependent vasodilatory function. The patterns by which sedentary time is accumulated may be more important than the total sedentary time on lower-limb arterial health. 


\section{PGC- $1 \alpha$ controls the basal expression of UPR ${ }^{\mathrm{mt}}$ genes in skeletal} muscle

\section{Slavin and D.A. Hood}

Muscle Health Research Centre, York University, Toronto, ON M3J 1P3, Canada.

PGC-1 $\alpha$, a transcriptional co-activator, is deemed to be the master regulator of mitochondrial biogenesis in mammalian skeletal muscle, coordinating the transcription of mitochondrial genes encoded by the nuclear genome. The biochemical and physiological effects of PGC- $1 \alpha$ ablation in muscle are extensively documented, revealing reductions in basal mitochondrial content and function, causing an impairment in oxidative capacity and exercise intolerance. More recently, work is being devoted to unravelling mechanisms responsible for the handling of acute mitochondrial stress upon exercise, wherein the accumulation of misfolded mitochondrial proteins temporarily can disrupt muscle homeostasis. In particular, the Mitochondrial Unfolded Protein Response (UPR $\left.{ }^{\mathrm{mt}}\right)$ is of great interest, because it is a cytoprotective program that is activated during stress to upregulate the expression of chaperones and proteases that alleviate proteotoxicity and preserve mitochondrial function. Evidence suggests that activation of these pathways may be a prerequisite for mitochondrial adaptations following exercise. However, the relationship between mitochondrial biogenesis, $\mathrm{UPR}^{\mathrm{mt}}$ signaling and whether PGC- $1 \alpha$ retains a role in the regulation of $\mathrm{UPR}^{\mathrm{mt}}$ gene expression remains to be characterized. Thus, to investigate this, hindlimb muscles extracted from WT $(n=3)$ and wholebody PGC-1 $\alpha \mathrm{KO}(\mathrm{n}=5)$ mice were used to measure mRNA levels using qPCR methods. The mRNA levels of PGC- $1 \alpha$ were completely abolished in the KO animals, and COX-IV mRNA was reduced by $65 \%$. The mRNA of downstream targets of UPR ${ }^{\mathrm{mt}}$ signaling, including the chaperone HSP60 and proteases LONP and ClpP were reduced by $75 \%, 60 \%$, and $89 \%$, respectively. Interestingly, the UPR ${ }^{\mathrm{mt}}$ transcription factor ATF5 was upregulated 2.5-fold in KO muscle, while CHOP, another stress-related transcription factor increased 20-fold, perhaps as a compensatory effort to rescue the lowered levels of UPR ${ }^{\mathrm{mt}}$ genes. No changes in ATF4 mRNA were observed in the absence of PGC- $1 \alpha$ expression. Our data indicate that PGC- $1 \alpha$, an influential effector of mitochondrial biogenesis in muscle, retains a regulatory function in coordinating the transcription of proteostatic genes, contributing to the regulation of organelle proteostasis in muscle.

\section{Flow-mediated dilation changes in response to interval and} continuous exercise in patients with coronary artery disease

K.M. Soave, ${ }^{1}$ V. Dizonno, ${ }^{2}$ P.I. Oh, ${ }^{3}$ J.M. Goodman, ${ }^{2,3,4}$ and K.D. Currie ${ }^{1}$

${ }^{I}$ Department of Kinesiology, Michigan State University, East Lansing, MI 48824, USA

${ }^{2}$ Faculty of Kinesiology \& Physical Education, University of Toronto, Toronto, ON M5S 2C9, Canada.

${ }^{3}$ University Health Network, Toronto Rehabilitation Institute, Toronto, ON M5G 2A2, Canada.

${ }^{4}$ Division of Cardiology, Mt. Sinai Hospital, Toronto, ON M5G 1X5, Canada.

High-intensity interval exercise (HIIE) is known to improve endothelial health in those with coronary artery disease (CAD). Assessing how flow-mediated dilation (FMD), a measure of endothelial function, changes from pre to post-exercise provides information about the exercise stimulus, which can be used to determine the optimal exercise prescription for this clinical population. The purpose of this study was to compare the change in brachial artery FMD in response to two different HIIE protocols and the standard exercise prescription in cardiac rehabilitation [moderate-intensity continuous exercise (MICE)] in patients with CAD. Ten individuals with CAD ( $65 \pm 5$ years; 7 males) completed MICE and HIIE protocols with long intervals and moderate-intensity recovery $\left(4^{\prime} 4\right)$ and short intervals with low-intensity recovery $\left(10^{\prime} 1\right)$ in randomized order on separate days. Brachial artery diameters were recorded using duplex ultrasound at baseline and for 3-minutes following a 5-minute period of forearm ischemia and were analyzed to determine FMD scaled to baseline diameter. FMD was assessed pre- and 60-minutes post-exercise and the change (D) in FMD was determined and compared between protocols using a repeated measured ANOVA. There was no difference in DFMD between protocols $\left(4^{\prime} 4:-0.29 \pm 0.70 \% ; 10^{\prime} 1:-0.34 \pm 0.61 \%\right.$; MICE: $-0.21 \pm 0.59 \% ; P=0.85$ ). While these averages indicate a decrease in FMD following all 3 protocols, individual responses were varied: $40 \%$ of the sample decreased FMD post-exercise following all 3 protocols, $30 \%$ decreased following 2 protocols, and 30\% decreased following 1 protocol. The observation of a similar DFMD between protocols suggests the two HIIE protocols and MICE provided a similar endothelial stimulus despite employing different exercise durations and intensities; however, examination of the individual responses to each exercise bout may help to identify the optimal prescription for each patient.

\section{Understanding individual variability in maximal cardiorespiratory fitness}

\section{S. Soni, J. Clarke, and R. Ross}

School of Kinesiology and Health Studies, Queen's University, Kingston, ON K7L 3N6, Canada.

Cardiorespiratory fitness as measured by maximal oxygen consumption $\left(\mathrm{VO}_{2}\right.$ peak) is a widely used measure in exercise science. However, several authors have demonstrated poor reliability for $\dot{\mathrm{VO}}_{2}$ peak measurements and have suggested single measurements prior and following an exercise intervention may not be sufficient, particularly when understanding the individual response to exercise. This study sought to better understand the individual variability between repeated $\dot{\mathrm{VO}}_{2}$ peak tests. Twenty-five adult men (age: $44.3 \pm$ 9.1 years [mean $\pm \mathrm{SD}$ ], BMI: $30.1 \pm 5.2 \mathrm{~kg} / \mathrm{m}^{2}$ ) completed a supervised exercise intervention wherein all participants exercised at $65 \%$ of their $\dot{\mathrm{V}} \mathrm{O}_{2}$ peak for 30 minutes, 5 times per week for 4 weeks. $\dot{\mathrm{VO}}_{2}$ peak was measured pre and post an exercise intervention. Two tests were performed at each time point within 3 days of one another, with a minimum of 24 hours rest between each test. At baseline, the mean value for $\dot{\mathrm{V}}_{2}$ peak for test 1 was $40.8 \mathrm{~mL} / \mathrm{kg} / \mathrm{min}$, and for test 2 it was $40.7 \mathrm{~mL} / \mathrm{kg} / \mathrm{min}$. However, the individual differences between the two $\dot{\mathrm{VO}}_{2}$ peak tests ranged from -7.1 to $+6.0 \mathrm{~mL} / \mathrm{kg} / \mathrm{min}$. The mean value for $\mathrm{V}_{2}$ peak following the exercise intervention for test 1 was $43.3 \mathrm{~mL} / \mathrm{kg} / \mathrm{min}$ and test 2 was $43.6 \mathrm{~mL} / \mathrm{kg} / \mathrm{min}$. The individual difference between the two $\mathrm{VO}_{2}$ peak tests ranged from -7.8 and $+5.8 \mathrm{~mL} / \mathrm{kg} / \mathrm{min}$. These observations suggest that the repeatability of $\mathrm{VO}_{2}$ peak measures based on group means is misleading as they may mask substantial individual differences between tests. These observations strongly support the recommendation that $\mathrm{VO}_{2}$ peak be measured at least twice both pre- and post-intervention in all research and healthcare settings.

\section{Arterial wall behaviour during spontaneous pre-mature ventricular contractions: A case study}

\section{K.A. Stevens, C.E. Athaide, and J.S. Au}

Department of Kinesiology and Health Sciences, University of Waterloo, Waterloo, ON N2L 3G1, Canada.

Carotid artery longitudinal motion (CALM) is a distinct multiphasic and bidirectional motion pattern that is unique to an individual, consisting of arterial wall displacements with (anterograde) and against (retrograde) the direction of blood flow. Available evidence points to CALM as a novel indicator of vessel wall health that varies with age and disease status. The etiological origins of the CALM pattern are unclear, particularly due the challenge of separating local pressure and flow stimuli from upstream cardiac and shear forces. This unique case study examines the regulation of CALM in a 29-year-old male with spontaneous premature ventricular contractions (PVC). Compared to pre-PVC, PVC carotid blood velocity decreased by $81 \%$ (96.8 to $18.4 \mathrm{~cm} / \mathrm{s}$ ), pulse pressure decreased by $69 \%$ ( 58 to $18 \mathrm{mmHg}$ ), and apical left ventricular (LV) rotation decreased by $59 \%\left(6.9\right.$ to $\left.2.8^{\circ}\right)$ due to reduced 
LV filling time. Anterograde longitudinal wall motion remained unchanged from the pre-PVC to PVC beat $(0.06 \mathrm{~mm})$, while retrograde motion was reduced by $91 \%$ ( 0.75 to $0.07 \mathrm{~mm}$ ). The post-PVC beat compensated for the decreased blood flow from the PVC beat and there were notable increases in retrograde displacement by $1757 \%(0.07 \mathrm{~mm}$ to $1.30 \mathrm{~mm})$, mean blood velocity by $670 \%(18.4 \mathrm{~cm} / \mathrm{s}$ to $141.6 \mathrm{~cm} / \mathrm{s}$ ), pulse pressure by $322 \%$ (18 $\mathrm{mmHg}$ to $76 \mathrm{mmHg}$ ), apical rotation by $136 \%\left(2.8^{\circ}\right.$ to $\left.6.6^{\circ}\right)$ and longitudinal strain by $100 \%(-11 \%$ to $-22 \%$ ). Anterograde wall motion negligibly increased by $0.1 \mathrm{~mm}$. There is seemingly little influence of either local or upstream factors on anterograde wall motion whereas changes in retrograde motion appear to mirror shifts in blood pressure, blood velocity and upstream cardiac movement, although the primary motion regulator remains unclear. This case study provides evidence against the role of blood velocity and supports the potential importance of cardiac mechanics and local pressure in regulating the longitudinal wall motion pattern. (Funded by NSERC.)

Increased daily dairy and dairy alternative beverage consumption and walking enhance integrated rates of muscle protein synthesis in healthy older women

T. Stokes, ${ }^{1}$ Y. Mei, ${ }^{1}$ F. Seo, ${ }^{1}$ J. McKendry, ${ }^{1}$ C. McGlory, ${ }^{2}$ and S.M. Phillips ${ }^{1}$

${ }^{I}$ Department of Kinesiology, McMaster University, ON L8S 4L8, Canada.

${ }^{2}$ School of Kinesiology and Health Sciences, Queen's University, ON K7L 3N6, Canada

The efficacy of dietary protein to stimulate muscle protein synthesis (MPS), the key process in maintaining muscle mass, is reduced with age and is thus a primary driver of sarcopenia. We hypothesized that twice-daily milk (whole or skim) consumption would increase MPS in older women relative to a non-dairy milk 'alternative' and that MPS would be enhanced by increasing daily physical activity levels (PA). Twenty-two older women were randomly assigned to one of three intervention groups: whole milk (WM; $n=7,69 \pm 3 \mathrm{y}$ ), skim milk (SM; n=7, $68 \pm 3$ y) or almond beverage (AB; $n=8,63 \pm 3$ y). During Phase 1 (days 1-3), participants consumed the RDA for protein $\left(0.8 \mathrm{~g}\right.$ protein $\left.\cdot \mathrm{kg}^{-1} \cdot \mathrm{d}^{-1}\right)$ and performed their habitual daily PA. During Phase 2 (days 4 to 7), participants maintained their habitual PA levels but consumed an intervention diet consisting of the standardized diet plus twice-daily beverages $(250 \mathrm{~mL}$ each) of either $\mathrm{WM}, \mathrm{SM}$, or $\mathrm{AB}$. Finally, during Phase 3 (days 8 to 10), participants continued to consume their intervention diet and increased their PA to 150\% of habitual levels. Deuterated water was ingested throughout the study, and muscle biopsies were taken on days 1, 4, 7 and 10 to measure integrated MPS. Daily MPS rates were not differentially affected by consumption of WM, SM or AB during Phase 2. However, when collapsed across intervention group, MPS was significantly increased from Phase 1 to Phase $2\left(+0.133 \% \cdot d^{-1}\right.$; $95 \%$ C.I., $\left.0.035-0.231\right)$ and further increased from Phase 2 to Phase $3\left(+0.289 \% \cdot \mathrm{d}^{-1}\right.$; $95 \%$ C.I., $\left.0.063-0.250\right)$. In conclusion, increasing consumption of milk or dairy beverages increased MPS; however, physical activity through walking further increased daily MPS rates in older women, irrespective of beverage consumed.

\section{Skeletal muscle CARM1 is required for a healthy, long life}

D.W. Stouth, T.L. vanLieshout, S.Y. Ng, A. Mikhail, E.K. Webb, R. Raziee, and V. Ljubicic

Department of Kinesiology, McMaster University, Hamilton, ON L8S 4L8, Canada.

Coactivator-associated arginine methyltransferase 1 (CARM1) is emerging as an important player in skeletal muscle biology. We sought to elucidate the role of CARM1 in mediating muscle mass and function, as well as in the induction and progression of the muscle atrophy program. To this end, we engineered CARM1 skeletal muscle-specific knockout (mKO) mice and employed distinct, but complementary models of muscle atrophy, including neurogenic muscle disuse, food deprivation, and the sarcopenia of aging. CARM1 mKO resulted in reduced muscle mass and myofiber cross-sectional area concomitant with dysregulated autophagic and atrophic signaling, which indicates the requirement of CARM1 for the maintenance of muscle biology. Interestingly, CARM1 deletion mitigated the progression of both denervation- and fasting-induced skeletal muscle atrophy as compared to wild-type (WT) mice. Key mechanistic findings revealed that CARM1 interacts with the master neuromuscular regulator AMPK and attenuates the expression and activity of its downstream autophagy and atrophy networks, including ULK1 ${ }^{\text {Ser555 }}$ and FOXO3 ${ }^{\text {Ser588 }}$, following neurogenic muscle disuse and food deprivation. Surprisingly, both male and female mKO mice have a significantly shorter lifespan versus their WT littermates, revealed by a $\sim 50 \%$ reduction in survival at 22-months-old, which is equivalent to $\sim 70$ years-old in humans. As such, we observed significantly reduced functional outcomes of integrative physiology in old mKO mice compared to old WT animals, such as strength and motor performance, which occurred coincident with altered muscle AMPK signalling. Taken together, these results indicate that skeletal muscle CARM1 is indispensable for maintaining muscle mass, function, and lifespan. Targeting the interplay between CARM1 and AMPK may offer a viable therapeutic strategy for combating lifelimiting muscle wasting conditions.

\section{Differential regulation of muscle and renal sympathetic outflow during stress in humans}

André L. Teixeira, Jordan B. Lee, Massimo Nardone, Jamie F. Burr, and Philip J. Millar

Department of Human Health and Nutritional Sciences, University of Guelph, Guelph, ON, Canada.

Muscle sympathetic nerve activity (MSNA) exhibits high interindividual variability at rest and during stress and is commonly unrelated to blood pressure (BP). Given the sympathetic nervous system can be regulated in an organ-dependent manner, MSNA variability could be paralleled by variability in sympathetic outflow directed to other organs that contribute to BP regulation (i.e., kidneys). We tested the hypothesis that changes in MSNA would be negatively associated with renal vasoconstrictor responses during stress. Beatto-beat BP, MSNA and renal blood velocity (RBV; Doppler ultrasound) were measured in 19 healthy young participants (five females) at rest and during isometric handgrip exercise (IHG; $2 \mathrm{~min}, 30 \%$ of maximum voluntary contraction) with post-exercise circulatory occlusion (PECO) to isolate the muscle metaboreflex. The cold pressor test (CPT; $2 \mathrm{~min}, \sim 4^{\circ} \mathrm{C}$ ) was employed as a non-exercise sympathoexcitatory stimulus by submerging the left hand in ice water. Stressors were randomly performed with a 10-min rest interval. Renal vascular resistance (RVR), an index of sympathetically mediated renal vasoconstriction, was calculated as mean $\mathrm{BP} / \mathrm{RBV}$. Resting RVR was unrelated to MSNA burst frequency $(r=0.31, P=0.20)$ or total MSNA ( $\mathrm{r}=0.24, \mathrm{P}=0.33)$. As expected, mean $\mathrm{BP}(\Delta 23 \pm 9 \mathrm{mmHg}$, $P<0.001)$, MSNA burst frequency $(\Delta 20 \pm 14$ bursts $/ \mathrm{min}, P<0.001)$, total MSNA $(\Delta 1220 \pm 752$ a.u., $P=0.001)$ and RVR $(\Delta 0.52 \pm 0.22 \mathrm{mmHg} / \mathrm{cm} / \mathrm{s}$, $P<0.01$ ) increased during IHG and remained elevated during PECO. During CPT, mean BP $(\Delta 19 \pm 10 \mathrm{mmHg}, P<0.001)$, MSNA burst frequency ( $\Delta 18 \pm 12$ bursts/min, $P<0.001)$, total MSNA ( $\Delta 1591 \pm 1416$ a.u., $P<0.001)$ and RVR $(\Delta 0.34 \pm 0.28 \mathrm{mmHg} / \mathrm{cm} / \mathrm{s}, P=0.01)$ were similarly increased. Despite neuro-cardiovascular variables being increased during all stressors, there were no relationships between changes in RVR and MSNA burst frequency (IHG: $r=-0.24, P=0.33$; PECO: $r=-0.10, P=0.69$ and CPT: $r=0.44, P=0.06$ ) or total MSNA (IHG: $\mathrm{r}=0.34, P=0.16$; PECO: $\mathrm{r}=-0.26, P=0.28$ and CPT: $\mathrm{r}=0.36, P=0.13$ ). These findings support the concept of differential regulation of muscle and renal sympathetic outflow during stress in young healthy humans. Funding: Natural Science and Engineering Research Council of Canada. 


\section{Hemodynamic response to blood flow restriction during} semi-recumbent cycling and in recovery

K.M.A. Thompson, A.M. Coates, and J.F. Burr

Human Health and Nutritional Sciences, University of Guelph, Guelph, ON NIG 2W1, Canada

Blood flow restriction (BFR) exercise alters blood inflow and outflow of active limbs. As blood redistribution and metabolite accumulation may augment cardiovascular stress, we sought to characterize the hemodynamic response of varied restriction pressures applied during exercise and recovery. Continuous heart rate (HR), systolic (SBP) and diastolic blood pressure (DBP) were measured via finger photoplethysmography, while maximal inferior vena cava diameter (IVCD) and enddiastolic volume (EDV) were measured to quantify venous return. Participants $(\mathrm{n}=13)$ cycled on a stress-echo bed $\left(45^{\circ}\right.$ torso angle) for $5 \mathrm{~min}$ at $60 \mathrm{~W} / 60 \mathrm{RPM}$, with tourniquets inflated to $0 \%$ (control), $50 \%$, $75 \%, 100 \%$ and $125 \%$ of individualized limb occlusion pressure (LOP). In visit 2, participants cycled for $5 \mathrm{~min}$, followed by $5 \mathrm{~min}$ of passive BFR using the same pressures. BFR resulted in large reductions in venous return during both exercise and recovery, with pressures of 100 and $125 \%$ LOP reducing IVCD $\sim 15 \%(p<0.05)$ and EDV $\sim 11 \%(p<0.01)$ during exercise, and 75\% LOP reducing IVCD $\sim 20 \%(p<0.001)$ and EDV $\sim 18 \%$ $(p<0.02)$ following exercise. During exercise, HR was elevated during all restriction pressures compared with control but not from each other (largest $\Delta=22 \mathrm{bpm}, 125 \%$ vs control). HR was highest and different from all other pressures during post-exercise BFR at 75\% LOP $(\Delta=11 \mathrm{BPM}$ vs. control). SBP was elevated while exercising with restriction (control: $129 \pm 34 \mathrm{mmHg}$ vs. $100 \%: 180 \pm 29 \mathrm{mmHg}$ ) but was unaffected by BFR in recovery. DBP was increased with BFR at $100 \%$ and $125 \%$ LOP during exercise (control:65 $\pm 8 \mathrm{mmHg}$ vs. 100\%:98 $\pm 19 \mathrm{mmHg}, 125 \%: 86 \pm 10 \mathrm{mmHg}$ ) and at $75 \%, 100 \%$ and $125 \%$ during the rest period (control:64 $\pm 9 \mathrm{mmHg}$ vs. 75\%:72 $111 \mathrm{mmHg}, 100 \%: 71 \pm 12 \mathrm{mmHg}, 125 \%: 73 \pm 11 \mathrm{mmHg}$ ). Elevated myocardial workload, estimated via the rate pressure product, occurred at $75 \%$ LOP during rest, and at $75 \%, 100 \%$ and $125 \%$ LOP while exercising. In conclusion, the hemodynamic responses to BFR are restriction-pressure dependent and differ depending on whether BFR is applied during or after exercise and the elevated HR and pressor response may lead to increased myocardial stress during comparable, light exercise and rest.

The effect of age, sex, and acute exercise on the autophagy-lysosome system in skeletal muscle

\section{Triolo, R. Kumari, and D.A. Hood}

Kinesiology and Health Science, Muscle Health Research Centre, York University, Toronto, Canada.

During aging, muscle fibre atrophy occurs due to decrements in protein synthesis and elevations in protein degradation. The autophagylysosome system is a degradation pathway that targets damaged components into autophagosomes, followed by delivery of these to the lysosomes for degradation. These processes are not well characterized in aged muscle, and less is understood about the impact of biological sex. Thus, we evaluated autophagy-lysosome markers in young (4-6 months) and aged (22-24 months) male and female mice. the upstream autophagy protein Beclin-1 and downstream autophagy protein ATG7 were not different in young and aged muscle However, both young and aged females had 2-fold more ATG7 protein than age-matched males. Aged animals had 60\% more p62, and $40 \%$ more LC3-I protein than young animals, but no differences were found in LC3-II protein, implicating elevated autophagic signaling in aged muscle. Young females had less p62 protein than young male counterparts, a difference abolished with age. LC3-I protein was $25 \%$ higher in aged vs young males, a finding not matched in the female group. LC3-II protein was $38-48 \%$ lower in young and old female, compared to male animals, suggesting that muscle from females has a lower basal autophagic breakdown than male counterparts, but aging elicits a similar elevation in autophagy. The lysosome proteins v-ATPase and Cathepsin B were both elevated with age in males and females, which may be explained by the higher levels of active nuclear TFEB protein driving their transcription. Acute exhaustive exercise reduced both LC3-I and p62 protein, but only in male animals, independent of age, but no change was observed in LC3-II in any group. These results imply that exercise induces autophagic degradation in male, but not female mice. However, in both male and female mice, acute exercise promoted nuclear TFEB localization. These data suggest that exercise can increase the signaling towards lysosomal biogenesis in both young and aged muscle, which may help alleviate the lysosomal dysfunction observed with age.

\section{Time course of ryanodine receptor fragmentation following} a session of sprint interval exercise

T.R. Tripp, ${ }^{1}$ B.P. Frankish, ${ }^{1,2}$ V.M.Y. Lun, ${ }^{3}$ J.P. Wiley, ${ }^{3}$ J. Shearer, ${ }^{1,4}$ R.M. Murphy, ${ }^{4,5}$ and M.J. MacInnis ${ }^{1}$

${ }^{I}$ Faculty of Kinesiology, University of Calgary, Calgary, AB T2N 1N4, Canada.

${ }^{2}$ Department of Biochemistry and Genetics, La Trobe Institute for Molecular Science, Melbourne, Vic, 3086, Australia.

${ }^{3}$ University of Calgary Sport Medicine Centre, Faculty of Kinesiology, University of Calgary, Calgary, AB T2N 1N4, Canada.

${ }^{4}$ Department of Biochemistry \& Molecular Biology, Cumming School of Medicine, University of Calgary, Calgary, AB T2N 1N4, Canada.

${ }^{5}$ Department of Physiology, Anatomy and Microbiology, School of Life Sciences, La Trobe University, Melbourne, Vic, 3086, Australia.

Fragmentation of the ryanodine receptor 1 (RyR1) is a potential mechanism through which sprint interval training (SIT) triggers mitochondrial biogenesis. We sought to characterize the time course of RyR1 fragmentation following a session of SIT and to determine if fragmentation was related to the relative abundance of mitochondrial oxidative phosphorylation (OXPHOS) proteins or antioxidant enzymes in the Vastus lateralis muscle. Ten healthy, recreationally-active males $(n=6)$ and females $(n=4)$ underwent a muscle biopsy before, and 3, 6, and $24 \mathrm{~h}$ after, a session of SIT (six $30 \mathrm{~s}$ 'all-out' intervals separated by $4.5 \mathrm{~min}$ of rest). Using semiquantitative Western blotting, we measured full-length RyR1 protein at all time points as well as baseline OXPHOS (complexes II-V), superoxide dismutase 2 (SOD2), and catalase protein contents. Full-length RyR1 was not significantly decreased at $3 \mathrm{~h}$ (mean \pm SD: $-25 \pm 45 \%$; $\mathrm{p}>0.05$ ); however, the reduction in full-length RyR1 was significant at $6 \mathrm{~h}(-38 \pm 38 \% ; \mathrm{p}<0.05)$ and $24 \mathrm{~h}$ post-exercise $(-30 \pm 48 \%$; $\mathrm{p}<0.05$ ). The timing of peak RyR1 fragmentation varied between participants but seemed to occur later than important post-exercise signalling events, and the magnitude of this response was not significantly correlated with OXPHOS nor antioxidant enzyme protein content ( $\mathrm{p}>0.05$ for all correlations). Our results suggest that RyR1 fragmentation does not initiate the signalling cascade for mitochondrial biogenesis and is more closely regulated by factors other than those investigated in this experiment. (Funded by the Natural Sciences and Engineering Research Council of Canada.)

\section{An evaluation of reactive balance control during divided attention: Not out of reach}

M.L. Trotman, M. Kennefick, S. Coughlin, P. van Donkelaar, and B.H. Dalton

School of Health and Exercise Sciences, The University of British Columbia Okanagan, Kelowna, British Columbia, Canada.

Reactive standing balance control following hand perturbations is important for everyday living as humans constantly encounter perturbations to the upper limb while performing functional tasks. Further, when two tasks are performed simultaneously through a dual-task paradigm, cognitive processing increases and performance on one or both tasks is often disrupted, owing to attentional resources being divided. The purpose here was to assess the effects of increased cognitive processing by way of a working memory task 
on whole-body balance responses to perturbations of the hand during a continuous voluntary reaching task. Sixteen participants $(22.9 \pm$ 4.5 years; 8 females, 8 males) stood and grasped the handle of a KINARM, a robotic-controlled manipulandum paired with an augmented visual display, and completed 10 trials of 100 continuous mediolateral arm movements at a consistent speed of one movement per second. Twenty anteroposterior hand perturbations were interspersed randomly throughout each trial (single-task). To divide attention and increase working memory processing, an auditory n-back task was performed during half of the trials (dual-task) and during quiet standing (cognitive-motor task). Peak centre of pressure (COP) displacement and velocity, time to COP displacement onset and peak, as well as peak hand displacement and velocity following the hand perturbation, were evaluated. N-back response times slowed by $8 \%$ and accuracy decreased by $11 \%$ during the dualtask compared with the cognitive-motor task $(\mathrm{p}<0.001)$. Only peak COP displacement following posterior perturbations increased by $8 \%$ during dual- than single-task $(\mathrm{p}=0.039)$, with no other differences detected. Hand peak displacement decreased by $5 \%$ during dualcompared to single-task $(\mathrm{p}=0.015)$. The main findings indicate that with increased cognitive processing, attentional resources were allocated from the cognitive task towards upper limb movements, while attentional resources for balance seemed unaltered likely owing to sensory reweighting of the balance system. (Supported by NSERC and CFI.)

Does exercise prescription using perceptual measures of intensity improve cardiorespiratory fitness in adults with spinal cord injury?

S.E. Valentino, ${ }^{1}$ M.J. Hutchinson, ${ }^{2}$ V.L. Goosey-Tolfrey, ${ }^{2}$ and M.J. MacDonald ${ }^{1}$

${ }^{I}$ Department of Kinesiology, McMaster University, Canada.

${ }_{2}^{2}$ Peter Harrison Centre for Disability Sport, School of Sport, Exercise and Health Sciences, Loughborough

University, UK

Perceptual measures of exercise intensity, such as Borg's ratings of perceived exertion (RPE) scale, can be used to prescribe, and monitor, exercise intensity. These perceptual methods are practical for use with both untrained and trained individuals who may have non-linear relationships between exercise workload and heart rate for a variety of reasons such as spinal cord injury (SCI). The purpose of this systematic review and meta-analysis was to analyse, and summarise, the effect of regulating exercise interventions with perceptual measures of intensity on cardiorespiratory fitness in adults with SCI. Four databases were searched for articles with a combination of keywords relating to the topics: SCI, perceptual measures of exercise intensity, and exercise interventions. Two independent reviewers assessed the studies for eligibility, followed by completion of data extraction and quality appraisal using the GRADEpro tool. Nine studies met the eligibility criteria, thereby including data from 95 adults with SCI representing both sexes and a diverse range of age, time since injury, lesion level, and lesion completeness. Participants completed various exercise modalities, predominantly arm cycle ergometry $(\mathrm{n}=7)$, for an average of $38 \mathrm{~min}$. (10-60min.) $\mathrm{x}$ 3 sessions per week (2-5 sessions/wk) for 10 weeks (5-18 weeks) in duration. Studies used either the Borg's 6-20 RPE scale $(n=2)$ or the Category Ratio 10 (CR10) scale $(n=7)$ for the prescription of intensity. A meta-analysis was performed to examine the effect of perceptually guided exercise interventions on the mean difference (MD) of peak oxygen uptake $\left(\mathrm{V}_{2}\right.$ peak) and peak power output (POpeak). $\dot{\mathrm{VO}}_{2}$ peak and POpeak improved after exercise interventions prescribed by perceptual measures of intensity, (MD: $2.92 \mathrm{ml} / \mathrm{kg} / \mathrm{min}$; 95\%CI: $1.30,4.54 ; P=0.0004$ and MD: $9.8 W ; 95 \%$ CI: 5.5, 14.3; $P<0.0001$, respectively) (GRADE ratings: "Low"). The evidence summarized and analysed in this review supports the use of perceptual prescription methods for exercise prescription in individuals with SCI. Supported by OGS.
A "Step-Ramp-Step" protocol to identify running power and speed at maximal lactate steady state

C.R. van Rassel, O.O. Ajayi, K.M. Sales, R.A. Azevedo, J.M. Murias, and M.J. MacInnis

Faculty of Kinesiology, University of Calgary, Calgary, AB T2N 1N4 Canada

Identification of the upper limit of sustainable exercise, or maximal lactate steady state (MLSS), requires multiple invasive laboratory visits. A previously established Step-Ramp-Step (SRS) protocol has been shown to accurately predict the power output (PO) associated with MLSS in cyclists with a single visit. The purpose of this investigation was to determine whether a modified SRS protocol could predict the running $\mathrm{PO}$ - using a commercially available wearable sensor (Stryd) - and running speed associated with MLSS. Twelve ( 5 female) trained runners $\left(30.1 \pm 4.7\right.$ years, $57.1 \pm 5.9 \mathrm{ml} \cdot \mathrm{kg}^{-1} \cdot \mathrm{min}^{-1}[$ mean $\left.\pm \mathrm{SD}]\right)$ performed a moderate step transition (MOD) on a treadmill $(4.3 \mathrm{mph}$ to $5.3 \mathrm{mph}$ ) and an incremental exercise test $(0.5 \mathrm{mph} / \mathrm{min})$ to exhaustion, followed 30 minutes later by a heavy step transition (HVY) at $\sim 50 \%$ of the speed between the lactate threshold (LT) and respiratory compensation point (RCP). LT and RCP were determined using ventilatory data from the incremental exercise test. Using the mean response time corrected $\mathrm{PO}$ and $\dot{\mathrm{VO}}_{2}$ associated with LT, $\mathrm{PO}$ and $\dot{\mathrm{VO}}_{2}$ associated with $\mathrm{HVY}$, and $\mathrm{VO}_{2}$ associated with RCP, the PO at RCP (i.e., the SRSpredicted MLSS PO) was determined by extrapolation. The SRSpredicted running speed at MLSS was determined with the same method, with speed in place of PO. On separate visits, participants performed multiple 30-minute constant-speed treadmill tests to determine MLSS. SRS-predicted running PO at MLSS $(246 \pm 38 \mathrm{~W})$ was not different than actual running PO at MLSS $(245 \pm 38 \mathrm{~W} ; \mathrm{p}=0.69)$, SRS-predicted running speed at MLSS $(7.4 \pm 0.6 \mathrm{mph})$ was different than actual running speed at MLSS $(7.7 \pm 0.8 \mathrm{mph}) ; \mathrm{p}=0.008)$. Additionally, the SRS-prediction using running PO yielded a stronger correlation $\left(\mathrm{R}^{2}=0.98\right.$ vs. 0.82$)$ and more accurate predictions (bias $=-0.3 \%$ vs. $-4.3 \%$ ) than the SRS-prediction using treadmill running speed. Overall, this treadmill-based SRS protocol allows MLSS to be accurately determined using running PO (Stryd) with a single laboratory visit. (Supported by NSERC, NSERC-CREATE We-TRAC training program, and Alberta Innovates.)

Exercise training prevents aberrant myeloid colony formation in mice with diet-induced obesity

\section{J.J. Vanhie, ${ }^{1}$ N. Collao, ${ }^{1}$ M. Ngu, ${ }^{1}$ and M. De Lisio ${ }^{1,2}$}

${ }^{I}$ School of Human Kinetics, University of Ottawa, Ottawa, ON K1N 6N5, Canada.

${ }^{2}$ Department of Cellular and Molecular Medicine, University of Ottawa, Ottawa, ON KIN 6N5, Canada.

Diet-induced obesity (DIO) is characterized by myeloid lineage inflammatory cell overproduction from hematopoietic stem cells (HSCs) in the bone marrow. In mice, exercise training prevents myeloid skewing during hematopoietic regeneration following bone marrow ionizing radiation injury due in part to maintenance of the cellular composition of the HSC niche. The role of exercise training in reducing myeloid skewing in DIO remains unknown. To determine the extent to which exercise training sustains normal HSC content, differentiation, and niche components in the context of DIO, male CBA mice $(n=40)$ were given control (9\%; D10012M; CON) or high-fat (45\%; D12451M; HFD) diets for 8 weeks, with the latter 4 weeks involving a treadmill exercise program (EX) or remained sedentary (SED). EX occurred $3 \mathrm{~d} \cdot \mathrm{wk}^{-1}$ at $10 \mathrm{~m} \cdot \mathrm{min}^{-1}$ for $25-45$ minutes and was surrounded by endurance tests. HFD had significantly higher body weight and adiposity compared to CON, and EX mice had significantly higher endurance performance compared to SED. Total HSC, long-term HSC, short-term HSC, common myeloid progenitor, and multipotent progenitor cell concentrations were significantly higher in HFD compared to CON irrespective of exercise training. Mesenchymal stromal cell concentration was significantly lower with exercise in CON. There was no difference in adipocyte progenitor, 
endothelial progenitor, endothelial, common lymphoid progenitor, or granulocyte-macrophage progenitor cell concentration with either EX or HFD. Total colony counts and granulocyte-erythrocyte-monocytemegakaryocyte (myeloid) progenitor colony forming unit (CFU) counts were both significantly lower in EX compared to SED without any effect of diet. Granulocyte-macrophage (myeloid) CFU counts were significantly lower in EX compared to SED and significantly higher in HFD compared to CON. Our data suggest DIO induces HSC subpopulation expansion while EX prevents myeloid skewing and reduces mesenchymal stromal cells that regulate HSC maintenance. Therefore, EX may reduce chronic inflammation in obesity by regulating HSC fate. (Funding provided by NSERC and AICR.)

\section{Skeletal muscle CARM1 regulates the integrative physiology of performance and exercise-induced signaling}

T.L. vanLieshout, ${ }^{1}$ D.W. Stouth, ${ }^{1}$ S.Y. Ng, ${ }^{1}$ I.A. Rebalka, ${ }^{2}$ T.J. Hawke, ${ }^{2}$ and V. Ljubicic ${ }^{1}$

${ }^{I}$ Department of Kinesiology, McMaster University, Hamilton, ON L8S 4L8, Canada.

${ }^{2}$ Department of Pathology and Molecular Medicine, McMaster University, Hamilton, ON L8S 4 L8, Canada.

Coactivator-associated arginine methyltransferase 1 (CARM1) catalyzes the methylation of arginine residues on target proteins. A mechanistic understanding of the role(s) of CARM1 in skeletal muscle biology is lacking. Here, we test the hypotheses that: 1) Skeletal muscle-specific CARM1 knockout (mKO) animals will have reduced strength, endurance, and motor function compared to their wildtype (WT) littermates, and 2) The acute gene expression responses in skeletal muscle to a single bout of exercise will be altered in CARM1 mKO versus WT animals. To address our first hypothesis, male 12-week-old mKO mice and WT littermates underwent a variety of functional tests. We found that mKO mice had significantly weaker maximum grip strength, increased fatigue, and slower ambulatory activity relative to their WT counterparts. Skeletal muscle arginine methylation status and methylation of CARM1 substrates were significantly different in mKO vs WT animals. Following this we randomly assigned littermates to one of three experimental groups: sedentary (SED), acute exercise (0AE), or acute exercise followed by 3 hours of recovery (3AE) to examine the differences between genotypes in response to acute exercise. mKO mice ran significantly less than their WT littermates. Exercise-induced AMP-activated protein kinase phosphorylation levels in skeletal muscle were similar between genotypes. However, peroxisome proliferator-activated receptor $\gamma$ coactivator-1 $\alpha$ (PGC-1 $\alpha$ ) protein levels were significantly higher in the mKO mice, as compared to WT animals. Furthermore, transcript levels of PGC-1 $\alpha$ increased at 3AE in the WT animals, however this response to exercise was not observed in mKO muscles. In conclusion, this ongoing study demonstrates that the removal of CARM1 in skeletal muscle negatively impacts measures of muscle function, as well as alters the molecular response of skeletal muscle to acute physical activity. Altogether this study serves to increase our understanding of CARM1 and its role in regulating skeletal muscle biology.

\section{Impact of a small change approach to weight loss on adipose} and lean mass in adults with overweight and obesity

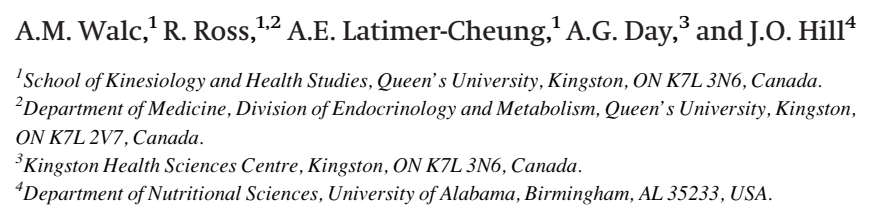

Efforts to manage obesity through weight loss have not been successful as most adults are unable to sustain the major changes in behaviour required to maintain weight loss long term. We sought to determine whether small changes in physical activity and diet prevent gains in weight and associated adiposity in adults with overweight and obesity. Previously inactive adults $(\mathrm{N}=289)$ were included in a secondary analysis of data derived from a three-year, single-centre, two-arm, longitudinal randomized controlled trial. Participants were randomized (1:1) to SCA ( $\mathrm{N}=144$, BMI: $32.4 \pm 4.2$ [mean $\pm \mathrm{SD}$ ], Age: $52.3 \pm 10.6 \mathrm{yrs}$ ) or monitoring alone (MA, N=145, BMI: $32.4 \pm 4.2$, Age: $53.1 \pm 10.6$ yrs). The SCA participants were counselled to make small changes in diet and physical activity, while MA participants were asked to maintain their usual lifestyle. Adiposity and lean mass were measured by dual-x-ray absorptiometry. Reduction in total adiposity was significantly greater in SCA than MA at 12 months (SCA: $-0.7 \pm 0.3$, MA: $-1.7 \pm 0.3, p=0.02$ ) but did not remain significant at 24 or 36 months $(\mathrm{p}>0.1)$. Reduction in visceral fat was significantly greater in SCA than MA at 6 months (SCA: $-2.1 \pm 0.4$, MA: $-0.4 \pm 0.4, \mathrm{p}=0.001$ ) but did not remain significant at 24 or 36 months ( $\mathrm{p}>0.1)$. There were no significantly different changes between groups in lean mass $(\mathrm{p}>0.1)$. The SCA did not prevent gains in adiposity or losses in lean mass compared to MA at two or three years in adults with overweight/obesity. No gain in adiposity or loss of lean mass was observed in either arm of the trial.

\section{MoveSTroNg at Home: A feasibility study of a model for remote delivery of functional strength and balance training combined with nutrition education for older pre-frail adults}

\section{E. Wang, ${ }^{1}$ H. Keller, ${ }^{1,2}$ and L. Giangregorio ${ }^{1,2}$}

${ }^{I}$ Department of Kinesiology, University of Waterloo, Waterloo, ON N2L 3G1, Canada. ${ }^{2}$ Schlegel-UW Research Institute for Aging, University of Waterloo, Waterloo, ON N2J OE2, Canada

MoveStrong was originally designed to provide a scalable framework to tailor fundamental movements for older adults. Through consultation with stakeholders, MoveStrong was adapted into an 8week remotely delivered exercise and nutrition education program for older pre-frail adults. The primary aim was to assess feasibility and acceptability. The secondary aim was to explore changes in physical function (3-point balance test, 30s chair stand test), physical activity (PAS), exercise self-efficacy (ESES), fatigue (CES-D), mental well-being (WEMWBS), quality of life and health status (EQ5D5L), nutritional risk (SCREEN@), dietary intake (ASA24) and record adverse events. We recruited community-dwelling Ontario residents; $\geq 60$ years, $\geq 1$ chronic condition, $\geq 1$ FRAIL scale. Participants received mailed materials, a personalized exercise program, 11 one-on-one sessions with an exercise physiologist via Physitrack ${ }^{\circledR}$, and three dietitian-led nutrition Q\&A sessions via Teams ${ }^{\circledR}$. We measured secondary outcomes and conducted participant experience interviews at program end and 4 weeks later. Participants were 74 (SD 7.29) years; 22 (73\%) participants were pre-frail, 8 (27\%) participants were frail. Exploratory analysis of secondary outcomes revealed significant improvements [program end, follow-up] in 30s chair stand test [3.5 (SD 6.1), 4.5 (SD 6.7)], physical activity [132 (SD 167), 82 (SD 150)], exercise self-efficacy [8.4 (SD 11.1), 9.7 (SD 12.1)], fatigue [0.70 (SD 1.17), 0.70 (SD 1.27)], health status [4.9 (SD 10.8), 9.1 (SD 11.9)], nutritional risk [10.0 (SD 5.4), 8.3 (SD 6.4)], and dietary protein intake [12.9 (SD 18.4), 9.2 (SD 22.7)]. No statistically significant changes occurred for other outcomes. 6 non-serious adverse events, not attributable to intervention, occurred. Overall participants were satisfied with the program and reported physical and psychological benefits. Barriers to maintenance were mapped to the transtheoretical domains of Environmental Context and Resources, and Social Influences (opportunity). (Supported by the Network of Aging Research.) 
Examining the impact of contraceptive hormones on the nitric oxide pathway in vascular endothelial cells

\section{J.S. Williams, M.J. Kamal, M. Wageh, G. Parise, and M.J. MacDonald}

Department of Kinesiology, McMaster University, Hamilton, ON L8S 4L8, Canada.

Oral contraceptive pills (OCP) are one of the most widely used prescriptions worldwide and are composed of ethinyl estradiol (EE) and one of several progestins (medroxyprogesterone acetate, MPA; levonorgestrel, LNG; desogestrel, DESO; drospirenone, DRP). In humans, the acute and chronic vascular responses to OCP seem to depend on the ratio of progestin to $\mathrm{EE}$ and the type of progestin, with some progestins (MPA, LNG) having indications of negative impacts on the vasculature. However, no research to date has examined the impact of EE and various progestins on vascular endothelial cells, specifically the endothelial nitric oxide (NO) production pathway. Therefore, the purpose of this study was to examine the impact of acute exposure of endogenous $17 \beta$ estradiol (E2), exogenous EE and various progestins (MPA, LNG, DESO, DRP) at physiologically relevant concentrations, on the NO production pathway in human umbilical vein endothelial cells. Following acute $24 \mathrm{~h}$ exposure to E2, EE or EE plus progestins, cell viability was unaltered; however, neither varying dosages of EE, nor E2, protected against oxidative stress-induced decrements in cell viability. Protein content of endothelial NO synthase (eNOS) and estrogen receptor (ER) $\alpha$ and $\beta$ were largely unchanged following acute exposure to EE, while serine 1177-phosphorylated eNOS (p-eNOS Ser1177) decreased at a low dosage of EE. Similarly, increasing doses of E2 resulted in decreases in ER $\alpha$ content, but not ER $\beta$, eNOS, or p-eNOS. Finally, combinations of EE and progestins did not alter either ER $\alpha / \beta$, eNOS, or p-eNOS. Future directions for this research will be to examine the functional production of intracellular NO and reactive oxygen species basally and in response to an acute acetylcholine challenge. This study fills a gap in our understanding of the cellular regulatory mechanisms underlying the impact of OCP on vascular endothelial function. (Supported by NSERC)

\section{More frequent breaks in sedentary time is associated with better} autonomic heart rate control in adults

\section{Y. Wu, M.W. O’Brien, J.A. Johns, J.L. Petterson, and D.S. Kimmerly}

Autonomic Cardiovascular Control and Exercise Lab, Division of Kinesiology, School of Health and Human Performance, Dalhousie University, Halifax, NS Canada.

Excessive overall time spent sedentary, and time spent engaged in prolonged sedentary bouts are independently associated with increased cardiovascular disease (CVD) risk. Heart rate variability (HRV) provides a marker of autonomic cardiac control. Reduced HRV can be indicative of sympathovagal imbalance, which is also related to higher CVD risk. However, the relationships between sedentary activity and sedentary patterns with HRV are currently unknown. The purpose of this project was to determine the relationship between habitual sedentary patterns and autonomic heart rate control in healthy adults. We hypothesized that decreased sedentary time, lower engagement in prolonged sedentary bouts and/ or more frequent sedentary breaks would be associated with higher vagal-based HRV indices. One hundred and nineteen healthy adults between 19-83 years (36 \pm 20 years, 67 females) wore an activPAL monitor on their right thigh for $\sim 7$ days to objectively measure daily sedentary patterns. At least 5-minutes of supine resting electrocardiogram recordings $(1000 \mathrm{~Hz})$ were used for HRV analyses. The root mean square of successive differences between normal heartbeats (RMSSD), normalized high-frequency power (HFnu), as well as the normalized low-frequency/HFnu ratio (sympathovagal balance) were calculated. Univariate and multiple regression analyses were used to examine the relationship between sedentary- and HRVrelated outcomes. In the multiple regression model, age $(\beta=-0.528$, $\mathrm{p}<0.001,95 \% \mathrm{CI}=-0.803,-0.253)$ and sedentary breaks per waking hour $(\beta=7.590, p=0.017,95 \% C I=1.399,13.781)$ were independent predictors of RMSSD. Sedentary breaks remained significant after partially controlling for all other significant predictors from the univariate analyses $(\mathrm{p}=0.017)$. There were no significant univariate relationships observed for any sedentary outcomes versus the HFnu or sympathovagal balance indices. More frequent sedentary breaks were related to healthier RMSSD values. Transitioning more often from sedentary postures to upright postures may provide an adaptive stimulus that enhances vagally-mediated cardiac control.

Does adjusting for mechanical work affect sex differences in peripheral and central fatigue during repeated sprints?

\section{S. Yoon, L.A. Cederbaum, and J.N. Côté}

Kinesiology and Physical Education, McGill University, Montreal, QC H2W 1S4, Canada.

Females generally demonstrate lower fatigability, and neuromuscular and hemodynamic adjustments during repeated sprints. However, these sex differences may be a consequence of the lower work performed by females than males during repeated sprint exercise. Accordingly, we examined sex differences in fatigue responses with and without adjustments for mechanical work during repeated sprints. Twenty recreationally active ( $>5 \mathrm{~h}$ of training/week), young adults (8 females) performed 10, 10-s sprints on a cycle ergometer, interspersed by 30 s of passive rest. Peripheral and central fatigue were quantified via changes in pre- to post-exercise potentiated quadriceps twitch force (evoked by supramaximal electrical stimulation of the femoral nerve) and quadriceps voluntary activation, respectively. Nearinfrared spectroscopy data were recorded during each sprint to quantify vastus lateralis oxygenation (deoxy[heme]) and were corrected for subcutaneous adipose tissue thickness (measured via ultrasonography). Blood lactate and strength (maximal isometric knee extension torque) were recorded pre- and post-exercise. These fatigue mechanisms were compared between the sexes with and without adjustments for total mechanical work of repeated sprints. Females experienced less peripheral fatigue ( $\mathrm{p}<.001)$, strength loss $(\mathrm{p}<.001)$, and muscle deoxygenation $(\mathrm{p}=.02)$. There were no sex differences in central fatigue and lactate increase $(\mathrm{p}>.05)$. Relative total work ( $\mathrm{J} / \mathrm{kg}$ of body mass) was comparable between the sexes $(\mathrm{p}=.63)$, while absolute total work (J) $(\mathrm{p}=.03)$, work decrement (i.e., fatigability) $(\mathrm{p}=.01)$, and initial work (first sprint) ( $\mathrm{p}=.01$ ) were higher in males compared to females. Adjusting for absolute and relative total work had no effects on the abovementioned sex comparison results. These data show that, independently from the amount of work performed, females still experience less peripheral adjustments in knee extensor muscles during repeated sprints. These results suggest sex dimorphism in fatigue resistance of knee extensor muscles. However, more participants will be tested to verify if this null finding is due to currently insufficient sample size.

Principles of exercise training in randomized controlled trials of cancers other than breast and prostate: An updated systematic review

K. Zadravec, ${ }^{1,2}$ K.A. Bland, ${ }^{3}$ S.E. Neil-Sztramko, ${ }^{4}$ M.E. Medysky, ${ }^{5}$ J. Kong, ${ }^{2}$ K.M. Winters-Stone, ${ }^{5}$ and K.L. Campbell ${ }^{2}$

${ }^{I}$ Rehabilitation Sciences Graduate Program, Faculty of Medicine, The University of British Columbia, Vancouver, BC V6T 1Z3, Canada.

${ }^{2}$ Department of Physical Therapy, The University of British Columbia, Vancouver, BC V6T IZ3, Canada. ${ }^{3}$ Mary MacKillop Institute for Health Research, Australian Catholic University, Melbourne, Victoria 3000, Australia. ${ }^{4}$ Department of Health Research Methods, Evidence, and Impact, McMaster University, Hamilton, ON L8S $4 K 1$, Canada

${ }^{5}$ Knight Cancer Institute, Oregon Health \& Science University, Portland, OR 97239-3098, USA.

Over the past decade, randomized controlled trials (RCTs) evaluating exercise's effect on cancer-related (e.g., survival), quality of life, and physical functioning outcomes have increased. To update our previous 
review of RCTs in cancers other than breast and prostate, we evaluate: (i) the application of exercise training principles (e.g., specificity, overload); (ii) reporting of exercise prescription components (i.e., FITT: frequency, intensity, time, and type); (iii) reporting of adherence to FITT components; and (iv) how reporting of FITT components/adherence changed over time. Eligible studies were RCTs evaluating the effect of aerobic and/or resistance exercise ( $\geq 4$ weeks) on physiological outcomes (e.g., aerobic capacity) in adults with cancers other than breast and prostate published from 2012-2020. A total of 107 studies were included. Cancer types were solid tumours (53\%), hematological cancers $(23 \%)$, and mixed diagnoses $(23 \%)$. Interventions were most commonly delivered after treatment for studies in solid tumours (46\%) and mixed diagnoses (44\%) and during treatment for studies in hematological cancers (52\%). The most frequently applied training principle was specificity (91\%), followed by initial values (72\%), overload (46\%), and progression (32\%). Few studies applied reversibility (7\%) or diminishing returns (5\%). In $58 \%$ of all studies, FITT components were clearly reported in the methods, although only $8 \%$ of all studies adequately reported adherence to these components. Compared to older studies, the percentage of studies published since 2011 that sufficiently reported initial values $(46 \%$ to $80 \% ; p<0.01)$ and progression $(15 \%$ to $37 \% ; p=0.039$ ) significantly increased. However, no improvements were found for reporting of FITT components/adherence in studies published since 2011 compared to older studies. Overall, our findings suggest improvements in exercise prescription design and reporting are still needed to better contextualize study results and enhance translation of evidence to practice.

Contralateral pain does not modulate corticospinal excitability or neuromuscular fatigue during repeated isometric knee extensions to task failure: Preliminary evidence

\section{J. Zhang, S. Abel, M. Macphail, and S.J. Aboodarda}

Faculty of Kinesiology, University of Calgary, Calgary, AB T2N 1N4, Canada.

Pain is an important determinant of exercise tolerance. Blood flow occlusion can simulate pain experienced during high-intensity exercise; yet, few studies investigated the effect of non-local muscle pain on neuromuscular fatigue and responses of the corticomotor system. We hypothesized that contralateral occlusion-induced pain would impede exercise tolerance by accelerating central fatigue, despite a reduction in peripheral fatigue and cortical inhibition. Seven participants ( 2 females; $27 \pm 7$ years) performed intermittent (6-s contraction:4-s rest) unilateral isometric knee extensions at $30 \%$ maximal voluntary contraction (MVC) to task failure (TF) with (OCCL) or without (CTRL) contralateral upper thigh occlusion. Electrical stimuli delivered to the femoral nerve assessed muscle excitability $\left(\mathrm{M}_{\text {max }}\right)$, evoked twitch force, and voluntary activation (VA). Single and paired pulse transcranial magnetic stimuli were used to assess corticospinal excitability [i.e., motor evoked potential (MEP) amplitude] and short interval intracortical inhibition (SICI), respectively. Every 12 contractions, rating of perceived exertion (RPE) and leg pain were measured, after which a MVC was performed with superimposed and resting twitches. Task duration was shorter in OCCL $(20 \pm 16 \mathrm{~min})$ than CTRL $(24 \pm 16 \mathrm{~min} ; P=0.003)$. Time effects were demonstrated in both conditions for MVC, VA, and resting twitch force. No significant effects were demonstrated in vastus lateralis MEP.M $\mathrm{M}_{\max }{ }^{-1}$, SICI (conditioned:unconditioned MEP), or $\mathrm{M}_{\max }$. RPE was higher across CTRL (16 \pm 6 vs. $14 \pm 4 ; P=0.013$ ) and continuously increased throughout both tasks before plateauing at $75 \%$. Contralateral leg pain was higher in OCCL at all timepoints $(P=0.015)$ while exercising leg pain was higher in CTRL at all timepoints $(P=0.015)$. Preliminary findings suggest that contralateral occlusive pain does not alter patterns of central or peripheral fatigue, corticospinal excitability, or $\mathrm{GABA}_{\mathrm{A}}$-mediated cortical inhibition during exhaustive intermittent isometric knee extensions. Despite a lack of neurophysiological differences, non-local perceived pain plays a strong role in reducing exercise tolerance. (Funded by the Natural Sciences and Engineering Research Council of Canada)

Higher body fat percentage in children with type 1 diabetes: a systematic review and meta-analysis

Y. Zheng, ${ }^{1}$ M. Rostami Haji Abadi, ${ }^{1}$ J. Gough, ${ }^{1}$ J.D. Johnston, ${ }^{2}$ M. Nour, ${ }^{3}$ and S.A. Kontulainen ${ }^{1}$

${ }^{I}$ College of Kinesiology, University of Saskatchewan, Saskatoon, SK S7N 5B2, Canada

${ }^{2}$ College of Engineering, University of Saskatchewan, Saskatoon, SK S7N 5A9, Canada

${ }^{3}$ College of Medicine, University of Saskatchewan, Saskatoon, SK S7N 5E5, Canada.

Children and adolescents with type 1 diabetes (DM1) are prone to overweight or obesity based on their higher BMI when compared to typically developing children (TDC). Differences in body composition, however, have not been systematically compared between children with DM1 and TDC. Our objective was to perform a systematic review and meta-analysis comparing total body fat and lean mass in children and adolescents with DM1 and TDC. We conducted literature searches in MEDLINE, Embase, SportDiscus, Web of Science and Scopus databases. We identified studies comparing DXAacquired total body fat and lean mass (absolute and percentage of body mass) between children with DM1 and TDC. We estimated mean differences between children with DM1 and TDC using a random-effects meta-analysis. We assessed heterogeneity by $\mathrm{I}^{2}$ and ran meta-regressions to explore the sources for heterogeneity. We included 21 studies of 1346 screened studies with 854 children and adolescents with DM1 and 898 TDC. Children with DM1 had 2.1\% higher body fat (95\%CI: 0.2 to $4.1 ; I^{2}=83 \%$ ). Insulin dosage (per $\mathrm{kg} / \mathrm{day}$ ) was positively associated with larger body fat percentage difference ( $\beta=15.4: 1.0$ to 29.7). There were no differences in fat and lean mass or lean mass percentage between children with DM1 and TDC $(P>0.05)$. This meta-analysis indicated $2 \%$ higher body fat percentage in children and adolescents with DM1. Insulin dosage was positively associated with body fat percentage difference between children with DM1 and TDC. More consideration to body fat percentage is warranted in clinical research and care of children with DM1 on higher daily insulin dosage. 